\title{
CUSP FORMS ON GSp(4) WITH SO(4)-PERIODS
}

\author{
YUVAL Z. FLICKER
}

\begin{abstract}
The Saito-Kurokawa lifting of automorphic representations from PGL(2) to the projective symplectic group of similitudes PGSp(4) of genus 2 is studied using the Fourier summation formula (an instance of the "relative trace formula"), thus characterising the image as the representations with a nonzero period for the special orthogonal group $\mathrm{SO}(4, E / F)$ associated to a quadratic extension $E$ of the global base field $F$, and a nonzero Fourier coefficient for a generic character of the unipotent radical of the Siegel parabolic subgroup. The image is nongeneric and almost everywhere nontempered, violating a naive generalization of the Ramanujan conjecture. Technical advances here concern the development of the summation formula and matching of relative orbital integrals.
\end{abstract}

1. Introduction. This paper concerns the determination of cusp forms on an adèle group $\mathbf{G}(\mathbb{A})$ whose period - namely integral - over a closed subspace ("cycle") arising from a subgroup $\mathbf{C}(\mathbb{A})$, is nonzero. Such forms contribute to the cohomology of the symmetric space $\mathbf{G} / \mathbf{C}$, and play a role in lifting automorphic forms to $\mathbf{G}(\mathbb{A})$ from another group $\mathbf{H}(\mathbb{A})$. Most advances in these studies so far have been made by means of the theory of the Weil representation [We]; see Waldspurger [Wa1/2], Howe and Piatetski-Shapiro [HPS], [PS], Kudla-Rallis [KR], Oda [O]. This technique has the advantage - in addition to early maturity - of constructing cusp forms on $\mathbf{G}(\mathbb{A})$ directly from such forms on $\mathbf{H}(\mathbb{A})$. Miraculously, the cusp forms on $\mathbf{G}(\mathbb{A})$ so obtained happen to have nonzero $\mathbf{C}(\mathbb{A})$-periods.

Our approach is based on a more naive and direct method, focusing more on the representation and its properties rather than on its particular realization. Thus we integrate both the spectral and the geometric expressions for the kernel $K_{f}(x, y)$ of the convolution operator on the space of cusp form on $\mathbf{G}(\mathbb{A})$, over the cycle associated with $\mathbf{C}(\mathbb{A})$. If both variables $x$ and $y$ are integrated over the cycle, one obtains a bi-period summation formula, involving the periods of the automorphic forms over the cycles (in Jacquet [J1], and later in $[\mathrm{FH}]$, this is named a "relative trace" formula, although there are no traces in that formula). The case where $\mathbf{G}(\mathbb{A})$ is $\mathbf{H}(\mathbb{A}) \times \mathbf{H}(\mathbb{A})$ and $\mathbf{C}(\mathbb{A})$ is $\mathbf{H}(\mathbb{A})$ embedded diagonally, coincides with that of the usual trace formula on $\mathbf{H}(\mathbb{A})$; this case is also referred to as the "group case".

Department of Mathematics, The Ohio State University, 231 W. 18th Ave., Columbus, OH 43210-1174; email: yzflicker@gmail.com

2000 Mathematics subject classification. Primary 11F70, 11F72; Secondary 22E35, 22E50, 22E55

Key words and phrases. Periods of automorphic forms, symplectic group, Saito-Kurokawa lifting, Fourier summation formula, relative orbital integrals, fundamental lemma 
If the second variable is integrated over a unipotent radical of a parabolic subgroup against an additive character, the Fourier summation formula - involving Fourier coefficients of the automorphic forms - is obtained (see Jacquet [J2], where the formula is again named "relative trace" formula, and $[\mathrm{F} 1 / 2 / 4])$. Only the cusp forms on $\mathbf{G}(\mathbb{A})$ with nonzero $\mathbf{C}(\mathbb{A})$-periods survive on the spectral side. The geometric side is compared with the geometric side of an analogous summation formula on $\mathbf{H}(\mathbb{A})$, for matching test functions on $\mathbf{G}(\mathbb{A})$ and $\mathbf{H}(\mathbb{A})$. The resulting identity of spectral sides can be used to establish lifting from $\mathbf{H}(\mathbb{A})$ to $\mathbf{G}(\mathbb{A})$. In summary, both the bi-period and the Fourier summation formulae are special instances of the "relative trace" formula.

The study of the Fourier summation formula, and the characterization of the relevant orbital integrals, lead to deep chapters in global, and local, harmonic analysis, especially of symmetric spaces; cf., e.g., [OM], [BS]. The analytic problems thus raised might even be considered to be of greater importance than the motivating final applications in representation theory. Conversely, these applications justify some of the work which has been done on symmetric spaces. One expects to derive identities of (bi-period or) Whittaker-Period distributions intrinsically related to the (local) representation in question. These distributions are analogous to Harish-Chandra's characters, which play a key role in studies of automorphic forms by means of the Selberg trace formula. To fully harvest the (bi-period, or) Whittaker-Period summation formulae, one would need an analogue of the orthogonality relations of characters, due to Harish-Chandra and Kazhdan $[\mathrm{K}]$, for these local distributions. The summation formula has been slow to evolve possibly since its application is based on panoply of techniques, substantially different from each other. Yet it could be a source of inspiration in various branches of contemporary harmonic analysis.

This paper focuses on an example, of automorphic forms on $\mathbf{G}=\mathrm{GSp}(4)$ and the cycle $\mathbf{C}=\mathbf{Z} \cdot \mathrm{SO}(4, E / F)$ associated to a quadratic separable extension $E$ of the global base field $F$. Here $Z, \mathbf{Z}(\mathbb{A})$ denote the centers of $G, \mathbf{G}(\mathbb{A})$. More precisely, $\mathbf{G}$ is the algebraic group of $g \in \mathrm{GL}(4)$ with $g J^{t} g=\lambda J, \lambda=\lambda(g) \in \mathrm{GL}(1), J=\left(\begin{array}{cc}0 & w \\ -w & 0\end{array}\right), w=\left(\begin{array}{ll}0 & 1 \\ 1 & 0\end{array}\right)$, and we put $G=\mathbf{G}(F) ; \mathbb{A}$ is the ring of adèles of $F$. We fix $\theta \in F^{\times}$which is not a square in $F$, put $\boldsymbol{\theta}=\left(\begin{array}{ll}0 & 1 \\ \theta & 0\end{array}\right)$ and $\Theta_{\theta}=\left(\begin{array}{ll}\boldsymbol{\theta} & 0 \\ 0 & \boldsymbol{\theta}\end{array}\right)$, and let $\mathbf{C}_{\theta}$ be the centralizer of $\Theta_{\theta}$ in $\mathbf{G}$. Put $C_{\theta}=\mathbf{C}_{\theta}(F)$. Also consider the unipotent radical $\mathbf{N}=\left\{n=\left(\begin{array}{cc}I & X \\ 0 & I\end{array}\right) ; X=\left(\begin{array}{cc}x & y \\ z & x\end{array}\right)\right\}$ of the Siegel parabolic subgroup $\mathbf{P}$ of type $(2,2)$ of $\mathbf{G}$, a complex valued nontrivial character $\boldsymbol{\psi}$ of the additive group $\mathbb{A} / F$, and the character $\psi_{\theta}(n)=\boldsymbol{\psi}(\operatorname{tr} T X)=\boldsymbol{\psi}(z-\theta y), T=\left(\begin{array}{rr}0 & 1 \\ -\theta & 0\end{array}\right)$.

Our main global achievement in this work is to advance the theory of the Fourier summation formula, namely develop such a formula by expanding geometrically and spectrally the integral of the kernel $K_{f}(n, h)$ of the standard convolution operator $r(f)$ (for a test function $f$ ) on the space of automorphic forms. In fact we multiply $K_{f}(n, h)$ by $\bar{\psi}_{\theta}(n)$, and integrate over $n \in N \backslash \mathbf{N}(\mathbb{A})$ and $h \in \mathbf{Z}(\mathbb{A}) C_{\theta} \backslash \mathbf{C}_{\theta}(\mathbb{A})$. The Fourier summation formula is recorded in Proposition 10. On the spectral side we truncate with respect to the group $G$, and show that this truncation does not affect the geometric side. Remarkable cancelations occur, making possible the derivation of the formula. On the geometric side we obtain a new type of orbital integrals of the form $\int_{N_{v}} \int_{C_{\theta, v}} f_{v}(n \gamma h) \bar{\psi}_{\theta}(n) d n d h$. 
Our summation formula for GSp(4) takes the following form. Suppose that $f=f_{1} * f_{2}^{*}$, where $f_{2}^{*}(g)=\bar{f}_{2}\left(g^{-1}\right)$, and $f_{1}, f_{2}$ are $\mathbb{K}$-finite elements of $C_{c}^{\infty}(\mathbf{G}(\mathbb{A}))$ which are spherical ( $K_{v}$-biinvariant) outside $V$ (a finite set of places containing the archimedean ones). Here $\mathbb{K}=\prod_{v} K_{v}$, and $K_{v}=\operatorname{GSp}\left(4, R_{v}\right), R_{v}$ being the ring of integers in $F_{v}$. Define $f^{\theta}=\otimes f_{v}^{\theta}$ by $f_{v}^{\theta}\left(g \Theta_{\theta} g^{-1} J\right)=\int_{C_{\theta v} / Z_{v}} f_{v}(g h) d h$. With $u=\lambda^{-1}\left(1-y z-\theta^{-1} x^{2}\right)$, define the local integrals by

$$
\Psi\left(\lambda, f_{v}^{\theta}\right)=|\theta|_{v}^{-2}|\lambda|_{v}^{-3} \int_{F_{v}^{3}} f_{v}^{\theta}\left(\left(\begin{array}{cccc}
0 & u & y & x \\
-u & 0 & -x & \theta z \\
-y & x & 0 & -\theta \lambda \\
-x & -\theta z & \theta \lambda & 0
\end{array}\right)\right) \bar{\psi}_{v}\left(-\lambda^{-1}(y+z)\right) d x d y d z
$$

and

$$
\Psi^{i}\left(f_{v}^{\theta}\right)=\int_{F_{v}} f_{v}^{\theta}\left(i\left(\begin{array}{cccc}
0 & u & 1 & 0 \\
-u & 0 & 0 & \theta \\
-1 & 0 & 0 & 0 \\
0 & -\theta & 0 & 0
\end{array}\right)\right) \bar{\psi}_{v}(u) d u .
$$

Put $\Psi\left(\lambda, f^{\theta}\right)=\prod_{v} \Psi\left(\lambda, f_{v}^{\theta}\right), \Psi^{i}\left(f^{\theta}\right)=\prod_{v} \Psi^{i}\left(f_{v}^{\theta}\right)$. Then the (finite) sum ("the geometric side")

$$
\sum_{\lambda \in F^{\times}} \Psi\left(\lambda, f^{\theta}\right)+\sum_{i= \pm} \Psi^{i}\left(f^{\theta}\right)
$$

is equal to the sum ("spectral side") of

$$
\sum_{\pi} m(\pi) \sum_{\Phi} W_{\psi_{\theta}}(\pi(f) \Phi) P(\bar{\Phi})
$$

where

$$
W_{\psi_{\theta}}(\Phi)=\int_{N \backslash \mathbf{N}(\mathbb{A})} \Phi(n) \bar{\psi}_{\theta}(n) d n, \quad P(\Phi)=P_{\theta}(\Phi)=\int_{\mathbf{Z}(\mathbb{A}) C_{\theta} \backslash \mathbf{C}_{\theta}(\mathbb{A})} \Phi(h) d h,
$$

and $\pi$ in (8.1) ranges over the equivalence classes of discrete spectrum representations of $\mathbf{G}(\mathbb{A})$, and

$$
\begin{aligned}
& \frac{1}{8} \sum_{\omega} \int_{i \mathbb{R}}\left[\sum_{\Phi} E_{\theta}\left(I\left(f,(1, \omega),\left(\frac{1}{2}, \zeta-\frac{1}{2}\right)\right) \Phi,(1, \omega),\left(\frac{1}{2}, \zeta-\frac{1}{2}\right)\right)\right. \\
& \left.\cdot \mathcal{L}^{V}\left(\omega^{-1}, \frac{1}{2}-\zeta\right) \cdot \overline{\mathcal{L}}_{V}\left(\gamma_{0},(1, \omega),\left(\frac{1}{2}, \zeta-\frac{1}{2}\right), \Phi\right)\right] d \zeta .
\end{aligned}
$$

The last sum ranges over the unitary characters $\omega$ of $\mathbb{A}^{\times} / F^{\times} U \mathbb{R}_{+}^{\times}$. The Eisenstein series is associated with the character $h=(a, b, \lambda / b, \lambda / a) \mapsto\left|a^{2} / \lambda\right|^{1 / 2}|a b / \lambda|^{\zeta-\frac{1}{2}} \omega(a b / \lambda)$ of the diagonal subgroup. The functions $\mathcal{L}^{V}$ and $\mathcal{L}_{V}$ are defined and studied in section 8. Here $\gamma_{0}$ represents the reflection (23). The sum (10.1), in which the brackets [.] are replaced by the absolute value $|\cdot|$, is convergent. 
The geometric side of the summation formula for GSp(4) is compared with the geometric side (recorded in Proposition 4) of the summation formula for $\operatorname{GSp}(2)=\mathrm{GL}(2)$. The latter is the equality of this geometric side (of Proposition 4) with the spectral side, recorded in Proposition 7. Our applications are derived from the resulting equality of spectral sides, for matching test functions.

Our main local achievement is in characterizing the functions of (2.2) thus obtained, by studying their asymptotic behavior as $\lambda$ ranges over $F_{v}$, especially near zero; see Proposition 3 . This study involves integration over a certain quadric in the affine 5 -space. We are led to Fourier analysis with respect to quadratic forms, involving Weil's factor $\gamma_{\psi}$. Underlying our computations is the stationary phase method, where we use the Morse Lemma. We discover that the asymptotic behavior of these Fourier orbital integrals is compatible with that of analogous Fourier orbital integrals obtained in the analysis of the Fourier summation formula for $N \backslash \mathrm{PGL}(2) / A, A=\left\{\left(\begin{array}{ll}* & 0 \\ 0 & 1\end{array}\right)\right\}, N=\left\{\left(\begin{array}{ll}1 & * \\ 0 & 1\end{array}\right)\right\}$; see Proposition 5 (due to Jacquet [J2]). We relate these Fourier orbital integrals on PGSp(4) and PGL(2), proving the existence of matching, in Corollary 5.1, comparing the summation formula for PGSp(4) (Proposition 10) with that of PGL(2) (Proposition 7, [F1], [J2]) in Propositions 10.3, 11.

In Proposition 8 we record the statement that naturally related spherical functions on PGSp(4) and PGL(2) have matching Fourier orbital integrals. The case of the unit elements in the Hecke algebras is proven in Proposition 6. The general case was proposed as a conjecture in an early draft of this work. It has then been proved in Zinoviev's OSU thesis, and published in $[\mathrm{Z}]$, using the case of the unit elements. It would be interesting to find an alternative proof of this "fundamental lemma", possibly based on a "symmetric space" analogue of the regular functions technique of [F6], which might reduce the spherical case to that of smooth test functions, or to that of the unit element in the Hecke algebra, which are analyzed here.

As an application of our summation formula and study of orbital integrals we recover a result of Piatetski-Shapiro [PS1], which in fact motivated our study. Let $\rho_{v}$ be an admissible representation of $\operatorname{PGL}\left(2, F_{v}\right)$, and $\zeta$ a complex number. Write $I\left(\rho_{v}, \zeta\right)$ for the $G_{v}=\operatorname{PGSp}\left(4, F_{v}\right)$-module on the space of $\phi: G_{v} \rightarrow \rho_{v}$ which satisfy

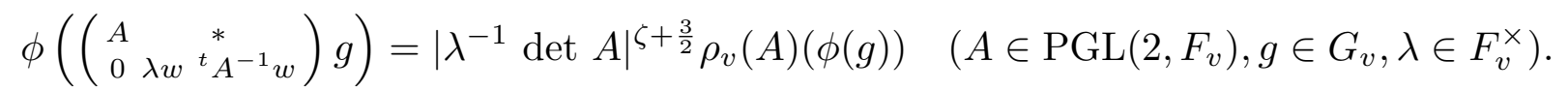

Write $J\left(\rho_{v}, \frac{1}{2}\right)$ for the Langlands' quotient (see [BW]) of $I\left(\rho_{v}, \frac{1}{2}\right)$ (it is unramified if so is $\rho_{v}$, and nontempered if $\rho_{v}$ is unitarizable). Proposition 12 asserts that if $\rho$ is a cuspidal representation of $\operatorname{PGL}(2, \mathbb{A})$ with $L\left(\frac{1}{2}, \rho \otimes \chi_{\theta}\right) \neq 0\left(\chi_{\theta}\right.$ is the quadratic character of $\mathbb{A}^{\times} / F^{\times}$associated with the quadratic extension $E=F(\sqrt{\theta})$ of $F)$ and $L\left(\frac{1}{2}, \rho\right)=0$, then there exists a cuspidal representation $\pi$ of $\operatorname{PGSp}(4, \mathbb{A})$ with $\pi_{v} \simeq J\left(\rho_{v}, \frac{1}{2}\right)$ for almost all $v$. This $\pi$ is $\mathbf{C}_{\theta}(\mathbb{A})$ cyclic, namely the period $P_{\theta}(\Phi)=\int_{\mathbf{Z}(\mathbb{A}) C_{\theta} \backslash \mathbf{C}_{\theta}(\mathbb{A})} \Phi(h) d h$ over the cycle $\mathbf{Z}(\mathbb{A}) C_{\theta} \backslash \mathbf{C}_{\theta}(\mathbb{A})$ is nonzero for some $\Phi \in \pi$, and is $\theta$-generic, namely $W_{\psi_{\theta}}(\Phi)=\int_{N \backslash \mathbf{N}(\mathbb{A})} \Phi(n) \bar{\psi}_{\theta}(n) d n$ is nonzero for some (possibly other) $\Phi \in \pi$. More precise local results could be obtained from our global theory had we had orthogonality relations for our Whittaker-Period distributions, analogous to those of Kazhdan $[\mathrm{K}]$ in the case of characters. By a cuspidal representation we mean an irreducible one. 
Conversely, Proposition 13 asserts that given a $\mathbf{C}_{\theta}(\mathbb{A})$-cyclic $\theta$-generic discrete spectrum representation $\pi$ of $\operatorname{PGSp}(4, \mathbb{A})$, either there exists a cuspidal $\operatorname{PGL}(2, \mathbb{A})$-module $\rho$ with $L\left(\frac{1}{2}, \rho \otimes \chi_{\theta}\right) \neq 0$ and $\pi_{v} \simeq J\left(\rho_{v}, \frac{1}{2}\right)$ for almost all $v$, or $\pi_{v} \simeq J\left(\chi_{\theta, v} \circ \operatorname{det}, \frac{1}{2}\right)$ for almost all $v$ (here $\chi_{\theta} \circ$ det is a one-dimensional, residual, discrete spectrum representation of $\operatorname{PGL}(2, \mathbb{A})$ defined by the quadratic character $\chi_{\theta}$ ). A brief discussion of the description of packets of such representations of $\operatorname{PGSp}(4, \mathbb{A})$ is given in the beginning of section 13 .

In section 14 we explain why there are no cusp forms on $\mathbf{G}(\mathbb{A})$ with periods by the split cycle $\mathrm{SO}(4)=\mathbf{C}_{0}=Z_{\mathbf{G}}\left(\left(\begin{array}{cc}\varepsilon & 0 \\ 0 & -\varepsilon\end{array}\right)\right), \varepsilon=\left(\begin{array}{cc}1 & 0 \\ 0 & -1\end{array}\right)$. For this reason we consider only $\mathrm{SO}(4, E / F)$-periods. In an Appendix we employ another form of the Fourier summation formula to study invariance of Fourier coefficients of cusp forms under the action of a certain stabilizer subgroup, recovering part of [PS2].

Under the isomorphism of $\mathrm{PGSp}(4)$ with $\mathrm{SO}(3,2)$, the image of $\mathbf{C}_{0}$ is the split group $\mathrm{SO}(2,2)$, and that of $\mathbf{C}_{\theta}$ is $\mathrm{SO}(3,1 ; E / F)$, the special orthogonal group associated with the sum of the hyperbolic form $x y$ and the norm form $z^{2}-\theta t^{2}$. Our techniques apply also with $G=\operatorname{PGSp}(2, D) \simeq \operatorname{SO}(4,1)$, where $D$ is a quaternion algebra, and $\mathbf{C}_{\theta}$ is again $\mathrm{SO}(3,1 ; E / F)$, if the field $E$ embeds in $D$. It would be interesting to study this situation, and its relation to the present work. Further, here we consider cyclic cusp forms with nonzero Fourier coefficients with respect to a generic character of the unipotent radical of the Siegel parabolic subgroup. There are no generic (with respect to a maximal unipotent subgroup) cyclic cusp forms on GSp(4). It would be interesting to consider also cyclic cusp forms generic with respect to the Heisenberg unipotent subgroup. Naturally it would be interesting to consider cusp forms on $\mathrm{SO}(n)$ cyclic with respect to $\mathrm{SO}(n-1)$, for a suitable choice of inner forms of these groups. The present work is a first step in this direction.

This paper is based on a preprint with the same title with J. G. M. Mars from the early 90's. I am grateful to him for teaching me lots of mathematics. Thanks are due to the referee for very careful reading. Support from the Humboldt Stiftung and MPIM-Bonn, and also the Fulbright Foundation, the Hebrew University and SFB at Universität Bielefeld, during the preparation of this work, is very much appreciated.

2. Definitions and notations. In this section we set the notations and recall the definitions we need (for a general connected reductive quasisplit group $G$ over $F$ ). We start with general definitions following the clear exposition of [A4], and then specialize to our case. So let $B$ be a minimal parabolic subgroup of $G$ over $F$. Fix a Levi subgroup $M_{B}$ of $B$. It is a torus over $F$ as $G$ is quasisplit. By a parabolic subgroup $P$ of $G$ over $F$ we shall mean one which is standard, namely containing $B$. Let $M_{P}$ be its unique Levi subgroup containing $M_{B}$, and $N_{P}$ its unipotent radical.

Let $A_{P}$ be the maximal split torus in the center of $M_{P}$. The group of rational characters of $A_{P}$ is $X^{*}\left(A_{P}\right)=\operatorname{Hom}\left(A_{P}, \mathrm{GL}(1)\right)$. Let $X_{*}\left(A_{P}\right)=\operatorname{Hom}\left(\mathrm{GL}(1), A_{P}\right)$ be the group of rational cocharacters of $A_{P}$. The map $X^{*}(G) \rightarrow X^{*}\left(A_{G}\right)$ is injective and has finite cokernel. For $G=\mathrm{GL}(n)$ this homomorphism is $x \mapsto n x, \mathbf{Z}(\mathbb{A}) \rightarrow \mathbf{Z}(\mathbb{A})$. We then obtain a canonical linear isomorphism

$$
\mathfrak{a}_{P}^{*}=X^{*}\left(M_{P}\right) \otimes \mathbb{R} \simeq X^{*}\left(A_{P}\right) \otimes \mathbb{R} .
$$

Let now $P_{1} \subset P_{2}$ be (standard) parabolics. We have embeddings $A_{P_{2}} \subset A_{P_{1}} \subset M_{P_{1}} \subset$ 
$M_{P_{2}}$. The restriction homomorphism $X^{*}\left(M_{P_{2}}\right) \rightarrow X^{*}\left(M_{P_{1}}\right)$ is injective. It yields a linear injection $\mathfrak{a}_{P_{2}}^{*} \hookrightarrow \mathfrak{a}_{P_{1}}^{*}$ and a dual linear surjection $\mathfrak{a}_{P_{1}} \rightarrow \mathfrak{a}_{P_{2}}$, where $\mathfrak{a}_{P}=X_{*}\left(A_{P}\right) \otimes$ $\mathbb{R} \simeq \operatorname{Hom}\left(X^{*}\left(A_{P}\right), \mathbb{R}\right)$. Denote the kernel of $\mathfrak{a}_{P_{1}} \rightarrow \mathfrak{a}_{P_{2}}$ by $\mathfrak{a}_{P_{1}}^{P_{2}} \subset \mathfrak{a}_{P_{1}}$. The restriction homomorphism $X^{*}\left(A_{P_{1}}\right) \rightarrow X^{*}\left(A_{P_{2}}\right)$ is surjective. It extends to a surjection $X^{*}\left(A_{P_{1}}\right) \otimes$ $\mathbb{R} \rightarrow X^{*}\left(A_{P_{2}}\right) \otimes \mathbb{R}$. We obtain a linear surjection $\mathfrak{a}_{P_{1}}^{*} \rightarrow \mathfrak{a}_{P_{2}}^{*}$, and a dual linear injection $\mathfrak{a}_{P_{2}} \hookrightarrow \mathfrak{a}_{P_{1}}$, hence split exact sequences of real vector spaces

$$
0 \rightarrow \mathfrak{a}_{P_{2}}^{*} \rightleftarrows \mathfrak{a}_{P_{1}}^{*} \rightarrow \mathfrak{a}_{P_{1}}^{*} / \mathfrak{a}_{P_{2}}^{*} \rightarrow 0
$$

and

$$
0 \rightarrow \mathfrak{a}_{P_{1}}^{P_{2}} \hookrightarrow \mathfrak{a}_{P_{1}} \rightleftarrows \mathfrak{a}_{P_{2}} \rightarrow 0
$$

Thus we have $\mathfrak{a}_{P_{1}}=\mathfrak{a}_{P_{2}} \oplus \mathfrak{a}_{P_{1}}^{P_{2}}$ and $\mathfrak{a}_{P_{1}}^{*}=\mathfrak{a}_{P_{2}}^{*} \oplus\left(\mathfrak{a}_{P_{1}}^{P_{2}}\right)^{*}$.

Given a parabolic $P$, let $\mathfrak{n}_{P}$ be the Lie Algebra of $N_{P}$. For $\alpha \in X^{*}\left(A_{P}\right)$ put

$$
\mathfrak{n}_{\alpha}=\left\{X_{\alpha} \in \mathfrak{n}_{P} ; \operatorname{Ad}(a) X_{\alpha}=\alpha(a) X_{\alpha}, a \in A_{P}\right\}
$$

The set of $\alpha$ with nonzero $\mathfrak{n}_{\alpha}$ is denoted by $\Phi_{P}$ and is called the set of roots of $A_{P}$ in $P$. It is a finite set of nonzero elements of $X^{*}\left(A_{P}\right)$ which parametrizes the decomposition $\mathfrak{n}_{P}=\oplus_{\alpha \in \Phi_{P}} \mathfrak{n}_{\alpha}$ of $\mathfrak{n}_{P}$ into eigenspaces under the adjoint action Ad: $A_{P} \rightarrow \mathrm{GL}\left(\mathfrak{n}_{P}\right)$ of $A_{P}$. Identify $\Phi_{P}$ with a subset of $\mathfrak{a}_{P}^{*}$ under the canonical maps $\Phi_{P} \subset X\left(A_{P}\right) \subset X\left(A_{P}\right) \otimes \mathbb{R} \simeq \mathfrak{a}_{P}^{*}$. If $H \in \mathfrak{a}_{G} \subset \mathfrak{a}_{P}$ then $\alpha(H)=0$ for every $\alpha \in \Phi_{P}$, so $\Phi_{P}$ lies in the subspace $\left(\mathfrak{a}_{P}^{G}\right)^{*}$ of $\mathfrak{a}_{P}^{*}$.

The pair $\left(V=\left(\mathfrak{a}_{0}^{G}\right)^{*}, R=\Phi_{0} \cup\left(-\Phi_{0}\right)\right)$, where $\left(\mathfrak{a}_{0}^{G}\right)^{*}$ is $\left(\mathfrak{a}_{P_{0}}^{G}\right)^{*}$ and $\Phi_{0}$ is $\Phi_{P_{0}}, P_{0}=B$, is a root system for which $\Phi_{0}$ is a system of positive roots. Write $W$ for the Weyl group of $(V, R)$. It is the group generated by the reflections about the elements in $\Phi_{0}$. It is a finite Coxeter group, hence has a length function $\ell$, and it acts on the vector spaces $V=\left(\mathfrak{a}_{0}^{G}\right)^{*}$, $\mathfrak{a}_{0}^{*}=\mathfrak{a}_{P_{0}}^{*}$, and $\mathfrak{a}_{0}=\mathfrak{a}_{P_{0}}$. Write $\Delta_{0} \subset \Phi_{0}$ for a basis: any $\beta \in \Phi_{0}$ can be written uniquely as $\sum_{\alpha \in \Delta_{0}} n_{\alpha} \alpha$ with integers $n_{\alpha} \geq 0$. The set $\Delta_{0}$ consists of the simple roots attached to $\Phi_{0}$, and $\Delta_{0}$ is a basis of the real vector space $V=\left(\mathfrak{a}_{0}^{G}\right)^{*}$. The set $\Delta_{0}^{\vee}=\left\{\alpha^{\vee} ; \alpha \in \Delta_{0}\right\}$ of simple coroots (defined by $\left\langle\alpha, \beta^{\vee}\right\rangle=2 \delta(\alpha, \beta)$ ) is a basis of the dual vector space $\mathfrak{a}_{0}^{G}=\mathfrak{a}_{P_{0}}^{G}$. Write $\widehat{\Delta}_{0}=\left\{\varpi_{\alpha} ; \alpha \in \Delta_{0}\right\}$ for the basis of $\left(\mathfrak{a}_{0}^{G}\right)^{*}$ dual to $\Delta_{0}^{\vee}$; its members are called the simple weights. Write $\widehat{\Delta}_{0}^{\vee}=\left\{\varpi_{\alpha}^{\vee} ; \alpha \in \Delta_{0}\right\}$ for the basis of $\mathfrak{a}_{0}^{G}$ dual to $\Delta_{0}$; its members are called the simple coweights.

Standard parabolic subgroups are parametrized by subsets of $\Delta_{0}$ : there is an order reversing bijection $P \leftrightarrow \Delta_{0}^{P}$ between standard parabolic subgroups $P$ of $G$ and subsets $\Delta_{0}^{P}$ of $\Delta_{0}$, such that

$$
\mathfrak{a}_{P}=\left\{H \in \mathfrak{a}_{0} ; \alpha(H)=0, \alpha \in \Delta_{0}^{P}\right\} .
$$

For any $P, \Delta_{0}^{P}$ is a basis of the space $\mathfrak{a}_{P_{0}}^{P}=\mathfrak{a}_{0}^{P}$. Let $\Delta_{P}$ be the set of linear forms on $\mathfrak{a}_{P}$ obtained by restriction of elements in the complement $\Delta_{0}-\Delta_{0}^{P}$ of $\Delta_{0}^{P}$ in $\Delta_{0}$. The set $\Delta_{P}$ is isomorphic to $\Delta_{0}-\Delta_{0}^{P}$, and any root in $\Phi_{P}$ can be written uniquely as a nonnegative integral linear combinations of elements $\Delta_{P}$. The set $\Delta_{P}$ is a basis of $\left(\mathfrak{a}_{P}^{G}\right)^{*}$. A second basis of $\left(\mathfrak{a}_{P}^{G}\right)^{*}$ is the subset $\widehat{\Delta}_{P}=\left\{\varpi_{\alpha} ; \alpha \in \Delta_{0}-\Delta_{0}^{P}\right\}$ of $\widehat{\Delta}_{0}$. Write $\Delta_{P}^{\vee}=\left\{\alpha^{\vee} ; \alpha \in \Delta_{P}\right\}$ for the 
basis of $\mathfrak{a}_{P}^{G}$ dual to $\widehat{\Delta}_{P}$, and $\widehat{\Delta}_{P}^{\vee}=\left\{\varpi^{\vee} ; \alpha \in \Delta_{P}\right\}$ for the basis of $\mathfrak{a}_{P}^{G}$ dual to $\Delta_{P}$. This notation is not standard if $P \neq P_{0}$. In this case, a general element $\alpha \in \Delta_{P}$ is not part of a root system (as defined in $[\mathrm{S}]$ ), so that $\alpha^{\vee}$ is not a coroot. Rather, if $\alpha$ is the restriction to $\mathfrak{a}_{P}$ of the simple root $\beta \in \Delta_{0}-\Delta_{0}^{P}, \alpha^{\vee}$ is the projection onto $\mathfrak{a}_{P}$ of the coroot $\beta^{\vee}$.

We have two bases $\Delta_{P}$ and $\widehat{\Delta}_{P}$ of $\left(\mathfrak{a}_{P}^{G}\right)^{*}$, and corresponding dual bases $\widehat{\Delta}_{P}^{\vee}$ and $\Delta_{P}^{\vee}$ of $\mathfrak{a}_{P}^{G}$, for any $P$. More generally, suppose that $P_{1} \subset P_{2}$ are two standard parabolic subgroups. Then we have two bases $\Delta_{P_{1}}^{P_{2}}$ and $\widehat{\Delta}_{P_{1}}^{P_{2}}$ of $\left(\mathfrak{a}_{P_{1}}^{P_{2}}\right)^{*}$, and corresponding dual bases $\left(\widehat{\Delta}_{P_{1}}^{P_{2}}\right)^{\vee}$ and $\left(\Delta_{P_{1}}^{P_{2}}\right)^{\vee}$ of $\mathfrak{a}_{P_{1}}^{P_{2}}$. The construction proceeds in the obvious way from the bases we have already defined. For example, $\Delta_{P_{1}}^{P_{2}}$ is the set of linear forms on the subspace $\mathfrak{a}_{P_{1}}^{P_{2}}$ of $\mathfrak{a}_{P_{1}}$ obtained by restricting elements in $\Delta_{0}^{P_{2}}-\Delta_{0}^{P_{1}}$, while $\widehat{\Delta}_{P_{1}}^{P_{2}}$ is the set of linear forms on $\mathfrak{a}_{P_{1}}^{P_{2}}$ obtained by restricting elements in $\widehat{\Delta}_{P_{1}}-\widehat{\Delta}_{P_{2}}$. We note that $P_{1} \cap M_{P_{2}}$ is a standard parabolic subgroup of the reductive group $M_{P_{2}}$, relative to the fixed minimal parabolic subgroup $P_{0} \cap M_{P_{2}}$. It follows from the definitions that

$$
\mathfrak{a}_{P_{1} \cap M_{P_{2}}}=\mathfrak{a}_{P_{1}}, \quad \mathfrak{a}_{P_{1} \cap M_{P_{2}}}^{M_{P_{2}}}=\mathfrak{a}_{P_{1}}^{P_{2}}, \quad \Delta_{P_{1} \cap M_{P_{2}}}=\Delta_{P_{1}}^{P_{2}}, \quad \widehat{\Delta}_{P_{1} \cap M_{P_{2}}}=\widehat{\Delta}_{P_{1}}^{P_{2}} .
$$

We now return to the group. There exists (see [MW], I.1.4) a maximal compact subgroup $\mathbb{K}$ of $G(\mathbb{A})$, fixed throughout this paper, satisfying: (1) $G(\mathbb{A})=B(\mathbb{A}) \mathbb{K} ;(2) P(\mathbb{A}) \cap \mathbb{K}=$ $\left(M_{P}(\mathbb{A}) \cap \mathbb{K}\right)\left(N_{P}(\mathbb{A}) \cap \mathbb{K}\right) ;(3) \mathbb{K} \cap M(\mathbb{A})$ is a maximal compact subgroup of $M(\mathbb{A})$ for all Levi subgroups $M \subset G$.

If $P_{1} \subset P_{2}$ are parabolic subgroups, let $\tau_{1}^{2}=\tau_{P_{1}}^{P_{2}}$ and $\widehat{\tau}_{1}^{2}=\widehat{\tau}_{P_{1}}^{P_{2}}$ be the characteristic functions on $\mathfrak{a}_{0}$ of $\left\{H \in \mathfrak{a}_{0} ;\langle\alpha, H\rangle>0, \alpha \in \Delta_{1}^{2}\right\}$ and $\left\{H \in \mathfrak{a}_{0} ;\langle\varpi, H\rangle>0, \varpi \in \widehat{\Delta}_{1}^{2}\right\}$.

For each parabolic subgroup $P$ in $G$ there is a Harish-Chandra map $\mathrm{H}_{P}: G(\mathbb{A}) \rightarrow \mathfrak{a}_{P}$ defined by:

(1) $|\chi|(m)=e^{\left\langle\chi, \mathrm{H}_{P}(m)\right\rangle}$ for all $m \in M_{P}(\mathbb{A})$ and $\chi \in X^{*}\left(M_{P}\right)$;

(2) $\mathrm{H}_{P}(n m k)=\mathrm{H}_{P}(m), n \in N_{P}(\mathbb{A}), m \in M_{P}(\mathbb{A}), k \in \mathbb{K}$.

For a group $G$, denote by $G(\mathbb{A})^{1}$ the kernel of the Harish-Chandra map $\mathrm{H}_{G}$.

For any subgroup $U$ of $G(\mathbb{A})$, put $U^{1}$ for $U \cap G(\mathbb{A})^{1}$.

For a parabolic subgroup with Levi decomposition $P=M N$, denote by $\rho_{P}$ the unique element in $\mathfrak{a}_{P}^{*}$ satisfying $e^{2\left\langle\rho_{P}, \mathrm{H}_{P}(m)\right\rangle}=\left|\operatorname{Ad}_{N}(m)\right|$. Here $\operatorname{Ad}_{N}(m)$ is the adjoint action of $m$ on the Lie algebra of $N$.

Let $F_{\infty}$ denote $F \otimes_{\mathbb{Q}} \mathbb{R}$. There is an isomorphism $\left(F_{\infty}^{\times}\right)^{r} \simeq A_{P}\left(F_{\infty}\right)$ (see [MW], I.1.11). Let $A_{P}^{+}$for $P \neq G$ and $A_{G}^{+}$be the intersections of the image of $\left(\mathbb{R}_{+}^{\times}\right)^{r}$ in $A_{P}\left(F_{\infty}\right)$ with $G(\mathbb{A})^{1}$ and $Z(\mathbb{A})$, respectively. The Harish-Chandra map $\mathrm{H}_{P}$ induces an isomorphism of $A_{P}^{+}$onto $\mathfrak{a}_{P}$ and we have the decomposition $M_{P}(\mathbb{A})=A_{G}^{+} \times A_{P}^{+} \times\left(M_{P}(\mathbb{A}) \cap G(\mathbb{A})^{1}\right)$. For $X \in \mathfrak{a}_{P}$, write $e^{X}$ for the unique element in $A_{P}^{+}$such that $\mathrm{H}_{P}\left(e^{X}\right)=X$. For $\lambda \in \mathfrak{a}_{P}^{*}$, write $e^{\lambda}$ for the character $p \mapsto e^{\left\langle\lambda, \mathrm{H}_{P}(p)\right\rangle}$ of $P(\mathbb{A})$.

For a suitable normalization of the Haar measures, we have (see [MW], I.1.13)

$$
\int_{G(\mathbb{A})^{1}} f(x) d x=\int_{N_{P}(\mathbb{A})} \int_{A_{P}^{+}} \int_{M_{P}(\mathbb{A}) \cap G(\mathbb{A})^{1}} \int_{\mathbb{K}} f(n a m k) e^{-2\left\langle\rho_{P}, \mathrm{H}_{P}(a)\right\rangle} d n d a d m d k .
$$


The Weyl group of $M_{B}$ in $G$ is $W=N_{G}\left(M_{B}\right) / M_{B}$, where $N_{G}\left(M_{B}\right)$ is the normalizer of $M_{B}$ in $G$. For a Levi subgroup $M$ in $G$, let $W_{M}=N_{M}\left(M_{B}\right) / M_{B}$ be the Weyl group of $M$. For two parabolic subgroups $P$ and $Q$ with Levi factors $M_{P}$ and $M_{Q}$ respectively, let $W(P, Q)$ be the set of elements $w \in W$ of minimal length in their class $w W_{M_{P}}$, such that $w M_{P} w^{-1}=M_{Q}$. The minimal length condition is equivalent to the condition $w \Delta_{G}^{P}=\Delta_{G}^{Q}$.

Let $\mathfrak{z}$ be the center of the universal enveloping algebra of the complexified Lie algebra of $G_{\infty}=\prod_{v} G\left(F_{v}\right)$. The product is over the archimedean places $v$ of $F$. A function $\phi(g)$ on $G(\mathbb{A})$ is called $\mathfrak{z}$-finite if there is an ideal $I$ in the algebra $\mathfrak{z}$ of finite codimension such that $I \cdot \phi=0$.

Let $\mathbb{K}$ be a maximal compact subgroup of $G(\mathbb{A})$ as above. The function $\phi$ is called $\mathbb{K}$-finite if the span of the functions $g \mapsto \phi(g k)(\forall k \in \mathbb{K})$ is finite dimensional.

The function $\phi(g)$ is called smooth if for any $g=g_{\infty} g_{f}$, with $g_{\infty} \in G_{\infty}$ and $g_{f} \in G\left(\mathbb{A}_{f}\right)$, there exist a neighborhood $V_{\infty}$ of $g_{\infty}$ in $G_{\infty}$ and a neighborhood $V_{f}$ of $g_{f}$ in $G\left(\mathbb{A}_{f}\right)$, and a $C^{\infty}$-function $\phi_{\infty}: V_{\infty} \rightarrow \mathbb{C}$, such that $\phi\left(g_{\infty} g_{f}\right)=\phi_{\infty}\left(g_{\infty}\right)$ for all $g_{\infty} \in V_{\infty}$ and $g_{f} \in V_{f}$.

In the function field case $\phi$ is called smooth if it is locally constant.

Fix an embedding $i_{1}: G \hookrightarrow \mathrm{GL}(n)$, and $i: G \hookrightarrow \mathrm{SL}(2 n), i(g)=\left(i_{1}(g), i_{1}(g)^{-1}\right)$. For $g \in G(\mathbb{A})$, write $i(g)=\left(g_{k l}\right)_{k, l=1, \ldots, 2 n}$. Set

$$
\|g\|=\prod_{v} \max \left\{\left|g_{k l}\right|_{v} ; k, l=1, \ldots, 2 n\right\}
$$

where the product is over all places $v$ of $F$. Then a function $\phi$ is called of moderate growth if there are $C, C^{\prime} \in \mathbb{R}_{>0}$ such that for all $g \in G(\mathbb{A})$ we have $|\phi(g)| \leq C\|g\|^{C^{\prime}}$. The definition of moderate growth does not depend on the choice of the embedding $i$. As in [MW], I.2.17 we say that a function $\phi: G(F) \backslash G(\mathbb{A}) \rightarrow \mathbb{C}$ is called an automorphic form if (1) $\phi$ is smooth and of moderate growth, (2) $\phi$ is right $\mathbb{K}$-finite, (3) $\phi$ is $\mathfrak{z}$-finite.

The space of automorphic forms is denoted by $\mathcal{A}(G)$. For a parabolic subgroup $P=N M$ the space of automorphic forms of level $P$, denoted by $\mathcal{A}_{P}(G)$, is the space of smooth right $\mathbb{K}$-finite functions

$$
\phi^{(P)}: N(\mathbb{A}) M(F) \backslash G(\mathbb{A}) \rightarrow \mathbb{C}
$$

such that for every $k \in \mathbb{K}$ the function $m \mapsto \phi^{(P)}(m k)$ is an automorphic form of $M(\mathbb{A})$. For $\phi^{(P)} \in \mathcal{A}_{P}(G)$ and a parabolic subgroup $Q \subset P$ with unipotent radical $N_{Q}$, the constant term is defined by:

$$
\phi_{Q}^{(P)}(g)=\int_{N_{Q}(F) \backslash N_{Q}(\mathbb{A})} \phi^{(P)}(n g) d n .
$$

When $P=G$ we will omit the superscript and write $\phi_{Q}$ instead of $\phi_{Q}^{(G)}$. Note that the definition of the constant term applies also for any locally integrable function on $N(\mathbb{A}) M(F) \backslash G(\mathbb{A})$, not only for automorphic forms. A function will be called cuspidal if its constant term is zero for all proper parabolic subgroups. A representation of $G(\mathbb{A})$ will be called cuspidal if it is irreducible and its representation space consists of cuspidal functions. For a cuspidal representation $\sigma$ of $M(\mathbb{A})$ denote by $\mathcal{A}_{P}(G)_{\sigma}$ the subspace of $\mathcal{A}_{P}(G)$ consisting of functions $\phi$ which are $A_{P}$-invariant and such that for every $k \in \mathbb{K}$ the function $m \mapsto \phi^{(P)}(m k)$ belongs to the space of $\sigma$. 
Let $F$ be a number field. From now on, to emphasize, we denote algebraic groups by bold face characters: $\mathbf{G}, \mathbf{P}, \mathbf{B}, \mathbf{N}, \ldots$, and their groups of rational points by $G, P, B, N, \ldots$ Let then $\mathbf{G}=\mathrm{GSp}(4)=\left\{g \in \mathrm{GL}(4) ; g J^{t} g=\lambda J, \lambda=\lambda(g) \in \mathrm{GL}(1)\right\}$, where $J=\left(\begin{array}{cc}0 & w \\ -w & 0\end{array}\right)$ and $w=\left(\begin{array}{ll}0 & 1 \\ 1 & 0\end{array}\right)$, be the group of symplectic similitudes of a 4 -dimensional space over $F$, a local or global field of characteristic other than two. Here ${ }^{t} g$ denotes the transpose of $g$. The form $J$ has the advantage that the upper triangular subgroup $\mathbf{B}$ of $\mathbf{G}$ is a minimal parabolic. The maximal parabolics which contain $\mathbf{B}$ are the Siegel parabolic $\mathbf{P}=\mathbf{P}_{1}=$ $\mathbf{M N}, \mathbf{N}=\left\{\left(\begin{array}{cc}I & X \\ 0 & I\end{array}\right) ; X=\left(\begin{array}{cc}x & y \\ z & x\end{array}\right)\right\}, \mathbf{M}=\left\{\left(\begin{array}{cc}A & 0 \\ 0 & \lambda w^{t} A^{-1} w\end{array}\right)=\left(\begin{array}{cc}A & 0 \\ 0 & \lambda \varepsilon A \varepsilon\end{array}\right)\right\}$, where $\varepsilon=\left(\begin{array}{cc}1 & 0 \\ 0 & -1\end{array}\right)$; and $\mathbf{Q}=\mathbf{P}_{2}=\mathbf{M}_{Q} \mathbf{N}_{Q}, \mathbf{M}_{Q}=\left\{\left(\begin{array}{ccc}a & 0 & 0 \\ 0 & A & 0 \\ 0 & 0 & \lambda / a\end{array}\right)\right.$; $\left.\operatorname{det} A=\lambda\right\}$, with unipotent radical $\mathbf{N}_{Q}=$ $\left\{\left(\begin{array}{cccc}1 & x & y & z \\ 0 & 1 & 0 & y \\ 0 & 0 & 1 & -x \\ 0 & 0 & 0 & 1\end{array}\right)\right\}$ which is an Heisenberg group with center $\mathbf{Z}_{Q}=\left\{\left(\begin{array}{llll}1 & 0 & 0 & z \\ 0 & 1 & 0 & 0 \\ 0 & 0 & 1 & 0 \\ 0 & 0 & 0 & 1\end{array}\right)\right\}$.

Here is the root diagram for PGSp (4). The angle between any adjacent rays in the graph is $\pi / 4$ :

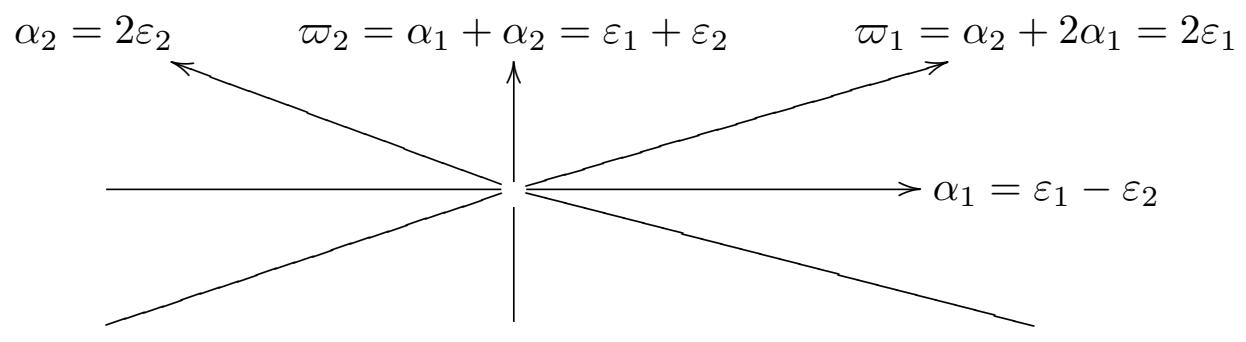

Here $\varepsilon_{1}=(1,0), \varepsilon_{2}=(0,1)$. The simple roots are $\alpha_{1}=\varepsilon_{1}-\varepsilon_{2}$, spanning $\mathfrak{a}_{2}^{*}=\mathfrak{a}_{0}^{1}$, and $\alpha_{2}=2 \varepsilon_{2}$, spanning $\mathfrak{a}_{1}^{*}=\mathfrak{a}_{0}^{2}$. The simple weights can be identified with $\varpi_{1}=\alpha_{2}+2 \alpha_{1}=2 \varepsilon_{1}$, spanning $\mathfrak{a}_{2}=\left(\mathfrak{a}_{0}^{1}\right)^{*}$, and $\varpi_{2}=\alpha_{1}+\alpha_{2}=\varepsilon_{1}+\varepsilon_{2}$, spanning $\mathfrak{a}_{1}=\left(\mathfrak{a}_{0}^{2}\right)^{*}$. We have $\mathrm{H}_{0}(\tilde{a} n k)=\mathrm{H}_{0}(\tilde{a})$. If $\tilde{a}=\operatorname{diag}(a, b, \lambda / b, \lambda / a)$, then

$$
\mathrm{H}=\mathrm{H}(\tilde{a})=\mathrm{H}_{0}(\tilde{a})=\ln \left|\frac{a}{b}\right| \cdot \varpi_{1}+\ln \left|\frac{b^{2}}{\lambda}\right| \cdot \varpi_{2}=\ln \left|\frac{a^{2}}{\lambda}\right| \cdot \alpha_{1}+\ln \left|\frac{a b}{\lambda}\right| \cdot \alpha_{2} .
$$

We have $\left.\mathrm{H}_{P}(d(A, \lambda))=\frac{3}{2} \ln |(\operatorname{det} A) / \lambda| \cdot \varpi_{2} \in \mathfrak{a}_{P}, \operatorname{as} \operatorname{Ad}_{N}(d(A, \lambda))=((\operatorname{det} A) / \lambda)\right)^{3}$. Also $\rho_{P} \in \mathfrak{a}_{P}^{*}$ is $\varepsilon_{2}=\frac{1}{2} \alpha_{2}$. Its projection to $\mathfrak{a}_{P}$ is $\frac{1}{2} \varpi_{2}$. That is, $\left\langle\frac{1}{2} \alpha_{2}, \varpi_{2}\right\rangle=\left\langle\frac{1}{2} \varpi_{2}, \varpi_{2}\right\rangle$.

The centralizer $\mathbf{C}=\mathbf{C}_{0}=Z_{\mathbf{G}}(\Theta)$ of $\Theta=\left(\begin{array}{cc}\varepsilon & 0 \\ 0 & -\varepsilon\end{array}\right)$ in $\mathbf{G}$ is

$$
\left\{\left(\begin{array}{llll}
a & 0 & 0 & b \\
0 & 0 & \beta & 0 \\
0 & \gamma & \delta & 0 \\
c & 0 & 0 & d
\end{array}\right) ; \operatorname{det}\left(\begin{array}{ll}
a & b \\
c & d
\end{array}\right)=\operatorname{det}\left(\begin{array}{ll}
\alpha & \beta \\
\gamma & \delta
\end{array}\right) \neq 0\right\} .
$$

Given $\theta \in F^{\times}$put $\boldsymbol{\theta}=\left(\begin{array}{ll}0 & 1 \\ \theta & 0\end{array}\right)$ and $\Theta_{\theta}=\left(\begin{array}{ll}\boldsymbol{\theta} & 0 \\ 0 & \boldsymbol{\theta}\end{array}\right)$. The centralizer $\mathbf{C}_{\theta}=Z_{\mathbf{G}}\left(\Theta_{\theta}\right)$ of $\Theta_{\theta}$ in $\mathbf{G}$ consists of the matrices $h=\left(\begin{array}{ll}a & b \\ c & d\end{array}\right), a=\left(\begin{array}{cc}a_{1} & a_{2} \\ \theta a_{2} & a_{1}\end{array}\right), \cdots, d=\left(\begin{array}{cc}d_{1} & d_{2} \\ \theta d_{2} & d_{1}\end{array}\right)$, such that $\left(\begin{array}{l}\mathbf{a} \mathbf{b} \\ \mathbf{c}\end{array}\right), \mathbf{a}=$ 
$a_{1}+a_{2} \sqrt{\theta}, \cdots, \mathbf{d}=d_{1}+d_{2} \sqrt{\theta}$, has determinant $\lambda(h)$ in GL(1). If $\theta \in F^{\times 2}$ is a square then $\Theta_{\theta}$ is conjugate to $\sqrt{\theta} \Theta$, and $\mathbf{C}_{\theta}$ is conjugate to $\mathbf{C}$. If $\theta \in F-F^{2}$ is not a square then $\mathbf{C}_{\theta}$ is isomorphic via the map just defined to $\{g \in \mathrm{GL}(2) / E$; $\operatorname{det} g \in \mathrm{GL}(1) / F\}$, where $E=F(\sqrt{\theta})$ is the quadratic extension of $F$ generated by the square root $\sqrt{\theta}$ of $\theta$. There is a natural injection $\mathbf{G} / \mathbf{C}_{\theta} \rightarrow \mathbf{X}(\theta), g \mapsto x=\frac{1}{\lambda} g \Theta_{\theta} J^{t} g=g \Theta_{\theta} g^{-1} J$, where

$$
\mathbf{X}(\theta)=\left\{x \in \mathbf{G} ; \lambda(x)=\theta,(x J)^{2}=\theta\right\}=\left\{x \in \mathbf{G} ; \lambda(x)=\theta,{ }^{t} x=-x\right\} .
$$

In particular, $\mathbf{G} / \mathbf{C} \rightarrow \mathbf{X}(1), g \mapsto x=\frac{1}{\lambda} g \Theta J^{t} g$, is an injection.

Fix a nontrivial additive character $\psi^{\prime}$ of $\mathbb{A} / F$. Let $\varepsilon$ be $\operatorname{diag}(1,-1)$. Define the character $\psi$ of $\mathbf{N}(\mathbb{A}) / N$ by $\psi(n)=\psi^{\prime}(\operatorname{tr}(\varepsilon \boldsymbol{\theta} X))$.

Denote by $\mathbf{A}$ the diagonal subgroup, and by $\mathbf{N}_{B}$ the unipotent upper triangular subgroup (thus $\mathbf{B}=\mathbf{A} \mathbf{N}_{B}$ ). Let $W=\operatorname{Norm}(\mathbf{A}) / \mathbf{A}$ be the Weyl group of $\mathbf{A}$ in $\mathbf{G}$, where $\operatorname{Norm}(\mathbf{A})$ is the normalizer of $\mathbf{A}$ in $\mathbf{G}$. Identify $W$ with a set of representatives $s$ in $\mathbf{G}$ with ${ }^{t} s=s^{-1}$ if $s$ is of order two in $W$. Double cosets decompositions as below are well known (see, e.g., $[\mathrm{Sp}])$.

Proposition 1. (a) Each $x$ in $\mathbf{X}(\theta)$ has the form $x=n s a^{t} n$ with $n \in \mathbf{N}_{B}, a \in \mathbf{A}, s \in W$ with $\left(s^{2}=1\right.$ in $W$ and $) s a=-a s^{-1}$.

(b) The group $\mathbf{G}$ is the disjoint union

$$
\mathbf{P C} \cup \mathbf{P} \gamma_{1} \mathbf{C}=\mathbf{B C} \cup \mathbf{B} \gamma_{1} \mathbf{C} \cup \mathbf{B} \gamma_{2} \mathbf{C} \cup \mathbf{B} \gamma_{3} \mathbf{C},
$$

where

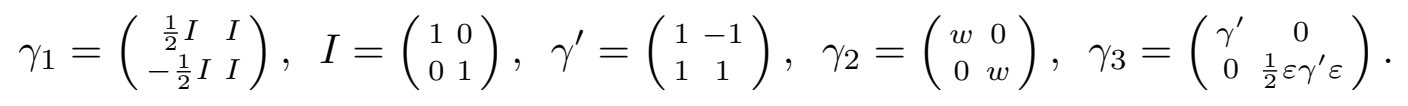

(c) If $\theta$ is not a square in $F$, then the group $G$ is the disjoint union $B C_{\theta} \cup B \gamma_{0} C_{\theta}$, and $\mathbf{G}$ is the disjoint union $\mathbf{P C}_{\theta} \cup \mathbf{P} \gamma_{0} \mathbf{C}_{\theta}, \gamma_{0}=\operatorname{diag}\left(1,\left(\begin{array}{cc}0 & 1 \\ -1 & 0\end{array}\right), 1\right)$.

(d) $\mathbf{G}$ acts on $\mathbf{X}(\theta)$ by $g: x \mapsto \frac{1}{\lambda(g)} g x^{t} g=g x J^{-1} g^{-1} J$. The variety $\mathbf{X}(\theta)$ consists of the points $\pm \sqrt{\theta} J$ which are fixed by $\mathbf{G}$, and the orbit

$$
\left\{\left(\begin{array}{cccc}
0 & a & b_{1} & b \\
-a & 0 & -b & b_{2} \\
-b_{1} & b & 0 & d \\
-b & -b_{2} & -d & 0
\end{array}\right) ; b_{1} b_{2}+b^{2}-a d=\theta\right\} \quad \text { of } \quad \Theta_{\theta} J .
$$

The stabilizer of $\Theta_{\theta} J$ is $\mathbf{Z}_{\mathbf{G}}\left(\Theta_{\theta}\right)=\mathbf{C}_{\theta}$, and of $\Theta J$ is $\mathbf{Z}_{\mathbf{G}}(\Theta)=\mathbf{C}$.

Proof. (a) Each $x$ in $\mathbf{G}$ can be expressed in the form $x=n_{1} s a^{t} n_{2}\left(a \in \mathbf{A}, s \in W, n_{i} \in \mathbf{N}_{B}\right)$. For $x$ in $\mathbf{X}(\theta)$ we have $s a=-a^{t} s$ (hence $s^{2}=1$ in $W$ ). We may assume that $n_{2}=1$, and write $n_{1}=n_{1}^{+} n_{1}^{-}$. Here $n_{1}^{+}$lies in the group $\mathbf{N}_{s}^{+}$which is generated by the root subgroups $\mathbf{N}_{\alpha}$ of $\mathbf{N}_{B}$ associated to the positive roots $\alpha$ such that $s \alpha$ is positive; $n_{1}^{-}$lies in the group $\mathbf{N}_{s}^{-}$which is generated by the $\mathbf{N}_{\alpha}$ with $\alpha>0$ and $s \alpha<0$. Then $n_{1}^{+} n_{1}^{-} s a=x={ }^{t} x=$ $s a^{t} n_{1}^{-t} n_{1}^{+}$, and so $n_{1}^{+}=1$ and $n_{1} \in \mathbf{N}_{s}^{-}$. The relation $n_{1} s a=s a^{t} n_{1}$ can be written as 
${ }^{t} n_{1}=a^{-1} s^{-1} n_{1} s a$, or on applying transpose and inverse, as $n_{1}^{-1}=a s^{-1 t} n_{1}^{-1} s a^{-1}$. Put $\sigma(n)=a s^{-1 t} n^{-1} s a^{-1}$. This is an automorphism of $\mathbf{N}_{s}^{-}$of order two. Since $\mathbf{N}_{s}^{-}$is a unipotent group, the first cohomology set $H^{1}\left(\langle\sigma\rangle, \mathbf{N}_{s}^{-}\right)$of the group $\langle\sigma\rangle$ generated by $\sigma$, with coefficients in $\mathbf{N}_{s}^{-}$, is trivial. This $H^{1}$ is the quotient of the set $\left\{n \in \mathbf{N}_{s}^{-} ; \sigma(n) n=1\right\}$ by the equivalence relation $n \equiv n_{0} n \sigma\left(n_{0}\right)^{-1}$. In particular, our $n_{1} \in \mathbf{N}_{s}^{-}$satisfies $n_{1} \sigma\left(n_{1}\right)=1$, hence it is in the equivalence class of 1 , namely there is $n \in \mathbf{N}_{s}^{-}$with $n_{1}=n \sigma(n)^{-1}$. Hence

$$
x=n_{1} s a=n \sigma(n)^{-1} s a=n a s^{-1 t} n s a^{-1} s a=n s a^{t} n,
$$

as required.

(b) The Weyl group $W$ of $\mathbf{G}$ consists of 8 elements, represented by the reflections 1, (14)(23), (12)(34), (13)(24), (14), (23), (2431), (3421). The last two are not of order 2, while for $s=1$ there are no $a \in \mathbf{A}$ with sas $=-a$. The transposition (23) is represented in $\mathbf{G}$ by $s=\operatorname{diag}\left(1,\left(\begin{array}{rr}0 & 1 \\ -1 & 0\end{array}\right), 1\right)$, but there is no $a \in \mathbf{A}$ with sas $=-a$. An analogous statement holds for (14).

Concerning the remaining 3 Weyl group elements, we have the following. Choose the representative $s_{4}=\left(\begin{array}{cc}0 & I \\ -I & 0\end{array}\right)$ for $(13)(24)$. If $x=s_{4} a, a \in \mathbf{A}$, then $x=-{ }^{t} x$ implies that $a=$ $\operatorname{diag}(b, b)$. Since $-J x=\left(\begin{array}{cc}w & 0 \\ 0 & w\end{array}\right) a$ has square $I$ we have that $b=\operatorname{diag}(c, 1 / c), c \in \operatorname{GL}(1)$. If $d=\operatorname{diag}(c, 1,1,1 / c)$ then $d s_{4}{ }^{t} d=s_{4} a$. Hence the part $\left\{n s_{4} a^{t} n ; n \in \mathbf{N}_{B}, a \in \mathbf{A}\right\}$ of $\mathbf{X}$ is equal to the $\mathbf{B}$-orbit $\lambda^{-1} b s_{4}{ }^{t} b$, which is the image of $\mathbf{B} \gamma_{3} \mathbf{C}$ under $\mathbf{G} / \mathbf{C} \rightarrow X, g \mapsto \lambda^{-1} g \Theta J^{t} g$.

As $s_{2}=J$ represents (14)(23), if $x=J a$ and $(J x)^{2}=I$, then the diagonal entries of $a \in \mathbf{A}$ are \pm 1 . Since $x=-{ }^{t} x$ implies that $J a=a J$, we have that $a= \pm I$ or $\pm \Theta$. Clearly there exists no $g \in \mathbf{G}$ such that $\lambda^{-1} g \Theta J^{t} g=g \Theta g^{-1} J$ is equal to $x=J a$ if $a= \pm I$. But when $g=I$ (resp. $g=\gamma_{2}$ ), then $x=J \Theta($ resp. $x=-J \Theta$ ) is obtained. We conclude that the part $\left\{n J a^{t} n ; n \in \mathbf{N}_{B}, a \in \mathbf{A}\right\}$ of $\mathbf{X}$ is the union of the $\mathbf{B}$-orbits $\pm \lambda^{-1} b J \Theta^{t} b$, namely the image of the union of the cosets $\mathbf{B C}$ and $\mathbf{B} \gamma_{2} \mathbf{C}$ under the map $\mathbf{G} / \mathbf{C} \rightarrow \mathbf{X}$.

Note that $\gamma_{1} J \Theta^{t} \gamma_{1}=s_{3} \Theta$ represents $(12)(34)$, where $s_{3}=\left(\begin{array}{cc}w & 0 \\ 0 & w\end{array}\right)$. If $x=s_{3} \Theta a$ then $x=-{ }^{t} x$ and $(J x)^{2}=I$ imply that $a=\operatorname{diag}(b, b, 1 / b, 1 / b)$, and $d=\operatorname{diag}(b, 1,1,1 / b)(b \in$ $\mathrm{GL}(1))$ satisfies $d J \Theta^{t} d=x$. Hence the part $\left\{n s_{3} \Theta a^{t} n ; n \in \mathbf{N}_{B}, a \in \mathbf{A}\right\}$ of $\mathbf{X}$ is the $\mathbf{B}$-orbit $\lambda^{-1} b s_{3} \Theta^{t} b$, which is the image of $\mathbf{B} \gamma_{1} \mathbf{C}$ under $\mathbf{G} / \mathbf{C} \rightarrow \mathbf{X}$.

The decomposition $\mathbf{G}=\mathbf{P C} \cup \mathbf{P} \gamma_{1} \mathbf{C}$ follows at once from the decomposition $\mathbf{B} \backslash \mathbf{G} / \mathbf{C}$. Note that we have proved also (d) in the case of $\theta=1$. The isolated points were obtained when $s_{2}=J$ was discussed; they are $x= \pm J$, not in the orbit of $\Theta J$.

(c) It suffices to consider $\theta \in F-F^{2}$. The proof follows closely that of (b). The relation $s a=-a s^{-1}$ implies that $s \neq 1,(14),(23)$, and the relation $(x J)^{2}=\theta$ implies that $s \neq(14)(23)$. Two elements of $W$ of order 2 are left.

Choose the representative $w_{0}^{\prime}=\operatorname{diag}\left(\left(\begin{array}{cc}0 & 1 \\ -1 & 0\end{array}\right),\left(\begin{array}{cc}0 & -1 \\ 1 & 0\end{array}\right)\right)$ for $(12)(34) \in W$. It satisfies ${ }^{t} w_{0}^{\prime} w_{0}^{\prime}=1$. It is more convenient though to work with $w_{0}=\operatorname{diag}\left(\left(\begin{array}{cc}0 & 1 \\ -1 & 0\end{array}\right),\left(\begin{array}{cc}0 & -\theta \\ \theta & 0\end{array}\right)\right)$. Since $w_{0}^{\prime-1}=-w_{0}^{\prime}$, we have that $x=x_{0} a=a w_{0}$. Then $a \in A$ has the form $a=\operatorname{diag}(b, b, c, c)$. From $\left(J w_{0} a\right)^{2}=\theta$ it follows that $b c=1$. But $d w_{0} d=w_{0} a=x$ if $d=\operatorname{diag}(b, 1,1,1 / b)$. 
Since $\gamma_{0} \Theta J^{t} \gamma_{0}=w_{0}$, the part $\left\{n a w_{0} a^{t} n ; n \in N_{B}, a \in A\right\}$ of $X(\theta)$ is the $B$-orbit $\lambda^{-1} b w_{0}{ }^{t} b$, which is the image of $B \gamma_{0} C_{\theta}$ under $G / C_{\theta} \rightarrow X(\theta)$.

Similarly we choose $\Theta_{\theta} J$ to represent $(13)(24) \in W$ (it is the product of $\operatorname{diag}(1, \theta, 1, \theta)$ and a representative $s \in W$ with ${ }^{t} s=s^{-1}=-s$ ). If $x=\Theta_{\theta} J a$ then the relation " $s a=$ $-a s^{-1} "$ implies that $a=\operatorname{diag}(b, c, b, c)$. Further, $b c=1$ from $(x J)^{2}=\theta$. But then $d \Theta_{\theta} J d=\Theta_{\theta} J a$ if $d=\operatorname{diag}(b, 1,1,1 / b)$. Hence the part $\left\{n a \Theta_{\theta} J a^{t} n ; a \in A, n \in N_{B}\right\}$ of $X(\theta)$ is the image of $B C_{\theta}$ under $G / C_{\theta} \rightarrow X(\theta)$. Then (c) follows, and so does (d).

3. Geometric side. Let $F$ be a global field (of characteristic $\neq 2$ ) $, \theta \in F-F^{2}, E=$ $F(\sqrt{\theta}), \mathbb{A}=\mathbb{A}_{F}$ and $\mathbb{A}_{E}$ the rings of adèles of $F$ and $E$. Put $G=\mathbf{G}(F), C_{\theta}=\mathbf{C}_{\theta}(F)$, and in general $Y=\mathbf{Y}(F)$ for any $F$-variety $\mathbf{Y}$. Denote by $L^{2}(\mathbf{Z}(\mathbb{A}) G \backslash \mathbf{G}(\mathbb{A}))$ the space of complex valued functions on $\mathbf{G}(\mathbb{A})$ which are left invariant under $G$ and $\mathbf{Z}(\mathbb{A})=\mathbf{Z}(\mathbb{A})$, where $\mathbf{Z}$ is the center of $\mathbf{G}$, and are absolutely square integrable on $\mathbf{Z}(\mathbb{A}) G \backslash \mathbf{G}(\mathbb{A})$. Let $f=\otimes f_{v}$ be a test function on $\mathbf{G}(\mathbb{A})$. Thus $f_{v} \in C_{c}^{\infty}\left(G_{v} / Z_{v}\right), G_{v}=\mathbf{G}\left(F_{v}\right)=\operatorname{GSp}\left(4, F_{v}\right)$, for all $v$, where $C_{c}^{\infty}$ means smooth (locally constant if $v$ is finite) and compactly supported. Moreover, for almost all $v$ the component $f_{v}$ is the unit element $f_{v}^{0}$ in the convolution algebra of $K_{v}=\mathbf{G}\left(R_{v}\right)$ biinvariant functions in $C_{c}^{\infty}\left(G_{v} / Z_{v}\right)\left(R_{v}\right.$ is the ring of integers in the nonarchimedean field $F_{v}$ ). Thus $f_{v}^{0}$ is the quotient of the characteristic function of $Z_{v} K_{v}$ in $G_{v}$ by the volume $\left|K_{v} Z_{v} / Z_{v}\right|$ with respect to the implicitly chosen Haar measure on $G_{v} / Z_{v}$. The convolution operator $(r(f) \phi)(h)=\int_{\mathbf{Z}(\mathbb{A}) \backslash \mathbf{G}(\mathbb{A})} f(g) \phi(h g) d g$ on $L^{2}(\mathbf{Z}(\mathbb{A}) G \backslash \mathbf{G}(\mathbb{A}))$ is an integral operator $\left(=\int_{\mathbf{Z}(\mathbb{A}) G \backslash \mathbf{G}(\mathbb{A})} K_{f}(h, g) \phi(g) d g\right)$ with kernel $K_{f}(g, h)=\sum_{\gamma \in Z \backslash G} f\left(g^{-1} \gamma h\right)$.

Our Fourier summation formula is based on integrating this kernel on $h$ in $\mathbf{Z}(\mathbb{A}) C_{\theta} \backslash \mathbf{C}_{\theta}(\mathbb{A})$ and $g$ in $N \backslash \mathbf{N}(\mathbb{A})$, against a character of $N \backslash \mathbf{N}(\mathbb{A})$, constructed as follows. Let $\boldsymbol{\psi}$ be a fixed nontrivial character of $\mathbb{A} / F$. The unipotent group $\mathbf{N}(\mathbb{A})$ consists of $n=\left(\begin{array}{ll}I & X \\ 0 & I\end{array}\right), X=\left(\begin{array}{ll}x & y \\ z & x\end{array}\right)$. Any character of $\mathbf{N}(\mathbb{A}) / N$ has the form $\psi_{T}(n)=\boldsymbol{\psi}(\operatorname{tr} T X)$, where $w^{t} T w=T$ is a $2 \times 2$ matrix with entries in $F$. The Levi subgroup $\mathbf{M}(\mathbb{A})$, consisting of $m=\operatorname{diag}\left(U, \lambda w^{t} U^{-1} w\right)$, acts on $\psi_{T}$ by

$$
\psi_{T}\left(m n m^{-1}\right)=\boldsymbol{\psi}\left(\lambda^{-1} \operatorname{tr} T U X w^{t} U w\right)=\boldsymbol{\psi}\left(\lambda^{-1} \operatorname{det} U \cdot \operatorname{tr} \varepsilon U^{-1} \varepsilon T U X\right) .
$$

Hence multiplying $U$, and consequently $m$, on the left by a suitable matrix, we may replace $\varepsilon T$ by a conjugate. Note that

$$
w^{t}\left(\varepsilon g^{-1} \varepsilon T g\right) w=\varepsilon g^{-1} \varepsilon T g \quad(g \in \mathrm{GL}(2)) .
$$

Moreover, the connected component $\operatorname{Stab}_{\mathbf{M}}^{0}\left(\psi_{T}\right)$ of the identity in the stabilizer $\operatorname{Stab}_{\mathbf{M}}\left(\psi_{T}\right)$ of $\psi_{T}$ in $\mathbf{M}$ is isomorphic to the centralizer $\mathbf{Z}(\varepsilon T)$ of $\varepsilon T$ in $\mathrm{GL}(2)$ via $m \leftrightarrow U, \lambda=\operatorname{det} U$. The centralizer $\mathbf{Z}(\varepsilon T)(\mathbb{A})$ is a torus in $\operatorname{GL}(2, \mathbb{A})$ when $\varepsilon T$ is nonsingular. Put $\psi_{\theta}$ for $\psi_{T}$ when $\varepsilon T=\left(\begin{array}{ll}0 & 1 \\ \theta & 0\end{array}\right), \theta \in F^{\times}$, and $\psi_{0}=\psi_{T}$ when $T=I$.

The absolute convergence of this integral is immediate. Let $\|g\|$ denote the usual norm function on the group $\mathbf{G}(\mathbb{A})$ ([HCM], p. 6). Then $\sum_{\gamma \in \mathbf{G}(F)}\left|f\left(g^{-1} \gamma h\right)\right| \leq c\|g\|^{N}$ (for some $c=c(f)>0, N=N(f)>0)$ for all $g, h$. Integrating the last sum over $h$ in the space $\mathbf{Z}(\mathbb{A}) C_{\theta} \backslash \mathbf{C}_{\theta}(\mathbb{A})$, which has finite volume, and over $g$ in the compact $\mathbf{U}(F) \backslash \mathbf{U}(\mathbb{A})$, where $\|g\|$ is bounded, we obtain a finite number.

We even have the following result (where we put $\mathbf{C}$ for $\mathbf{C}_{\theta}$ ). 
Lemma 1.1. If $f$ is compactly supported on $\mathbf{Z}(\mathbb{A}) \backslash \mathbf{G}(\mathbb{A})$, then the function $K_{f}$ is compactly supported on $N \backslash \mathbf{N}(\mathbb{A}) \times C \mathbf{Z}(\mathbb{A}) \backslash \mathbf{C}(\mathbb{A})$.

Proof. If $\mathbf{G}$ is a connected linear algebraic group over $F$, and $\mathbf{C}$ is a reductive closed subgroup over $F$, then $\mathbf{G} / \mathbf{C}$ is an affine variety $\mathbf{V}$ over $F$ ([Bo], Proposition 7.7). Then $V=$ $\mathbf{V}(F)$ is discrete and closed in $\mathbf{V}(\mathbb{A})$. The natural map $\mathbf{G}(\mathbb{A}) / \mathbf{C}(\mathbb{A}) \rightarrow \mathbf{V}(\mathbb{A})$ is continuous, and it maps $G / C \subset \mathbf{G}(\mathbb{A}) / \mathbf{C}(\mathbb{A})$ to $V \subset \mathbf{V}(\mathbb{A})$. Hence $G / C$ is closed in $\mathbf{G}(\mathbb{A}) / \mathbf{C}(\mathbb{A})$, namely $G \mathbf{C}(\mathbb{A})$ is closed in $\mathbf{G}(\mathbb{A})$ and so $\mathbf{C}(\mathbb{A}) / C$ is closed in $\mathbf{G}(\mathbb{A}) / G$. Moreover, for $G$ over $F$ as above, for any closed $F$-subgroup $H$ of $G, \mathbf{H}(\mathbb{A}) / H$ is closed in $\mathbf{G}(\mathbb{A}) / G([\mathrm{G}],(2.1))$. Now for our function $f$, since $N \backslash \mathbf{N}(\mathbb{A})$ is compact, $K_{f}(u, h)=\sum_{Z \backslash G} f\left(u^{-1} \gamma h\right)$ has compact support on $N \backslash \mathbf{N}(\mathbb{A}) \times G \mathbf{Z}(\mathbb{A}) \backslash \mathbf{G}(\mathbb{A})$, hence also on its closed subset $N \backslash \mathbf{N}(\mathbb{A}) \times C \mathbf{Z}(\mathbb{A}) \backslash \mathbf{C}(\mathbb{A})$, by either of these results.

A computational proof is as follows. The kernel $K_{f}\left(u^{-1}, h\right)=\sum_{\gamma \in G / Z} f(u \gamma h)$ is equal to $\Sigma_{\mu} \Sigma_{\eta} \Sigma_{\nu} f(u \nu \mu \eta h)\left(\mu \in N \backslash G / C, \eta \in C / Z, \nu \in N / N \cap \mu C \mu^{-1}\right)$. By Proposition 1(c), a set of representatives for the $\mu$ is given by the elements

(1) $\operatorname{diag}\left(\left(\begin{array}{cc}\alpha & \beta \\ 0 & 1\end{array}\right),\left(\begin{array}{cc}\alpha & -\beta \\ 0 & 1\end{array}\right)\right), \alpha \in F^{\times}, \beta \in F$; and (2) $\operatorname{diag}(1,1, \lambda, \lambda) \gamma_{0}, \lambda \in F^{\times}$.

If $u$ lies in a fixed compact subset of $\mathbf{N}(\mathbb{A})$, and $f(u \nu \mu \eta h) \neq 0$, then $\nu \mu \eta h$ lies in a compact subset of $\mathbf{G}(\mathbb{A}) / \mathbf{Z}(\mathbb{A})$. Hence $\operatorname{Ad}(\nu \mu) \Theta=\operatorname{Ad}(\nu \mu \eta h) \Theta$ stays in a compact of $\mathbf{G}(\mathbb{A})$, and consequently in a finite set. Put $\nu=\left(\begin{array}{ll}I & X \\ 0 & I\end{array}\right)$. Then for $\mu$ of the form (1), we have

$$
\nu \mu \Theta \mu^{-1} \nu^{-1}=\left(\begin{array}{cc}
A \boldsymbol{\theta} A^{-1} & -A \boldsymbol{\theta} A^{-1} X-X \varepsilon A \boldsymbol{\theta} A^{-1} \varepsilon \\
0 & -\varepsilon A \boldsymbol{\theta} A^{-1} \varepsilon
\end{array}\right), \quad A=\left(\begin{array}{cc}
\alpha & \beta \\
0 & 1
\end{array}\right), \boldsymbol{\theta}=\left(\begin{array}{ll}
0 & 1 \\
\theta & 0
\end{array}\right) .
$$

Since $A \boldsymbol{\theta} A^{-1}$ lies in a finite set, so do $\alpha$ and $\beta$. For $\mu$ of the form (2) we have

$$
\nu \mu \Theta \mu^{-1} \nu^{-1}=\left(\begin{array}{cc}
-\theta \lambda X \varepsilon & \theta \lambda X \varepsilon X-\lambda^{-1} \varepsilon \\
-\theta \lambda \varepsilon & \theta \lambda \varepsilon X
\end{array}\right) .
$$

Hence $\lambda$ lies in a finite set. Consequently only finitely many $\mu$ occur, and for each $\mu$ we have a summation over $\nu$ in a finite subset of $N / N \cap \mu C \mu^{-1}=\operatorname{Ad}(N) \mu \Theta \mu^{-1}$. Finally, since $\nu \mu \eta h$ lies in a compact of $\mathbf{G}(\mathbb{A}) / \mathbf{Z}(\mathbb{A})$, we conclude that $h$ stays in a compact set modulo $C \mathbf{Z}(\mathbb{A})$.

The geometric side of the Fourier summation formula is described as follows.

Proposition 2. For any $f=\otimes f_{v}$ on $\mathbf{G}(\mathbb{A})$ define $f^{\theta}=\otimes f_{v}^{\theta}$ on $\mathbf{X}(\theta)(\mathbb{A})$ by

$$
f_{v}^{\theta}\left(g \Theta_{\theta} g^{-1} J\right)=\int_{C_{\theta v} / Z_{v}} f_{v}(g h) d h .
$$

Then

$$
\int_{N \backslash \mathbf{N}(\mathbb{A})} \int_{\mathbf{Z}(\mathbb{A}) C_{\theta} \backslash \mathbf{C}_{\theta}(\mathbb{A})} \sum_{\gamma \in Z \backslash G} f\left(n^{-1} \gamma h\right) \bar{\psi}_{\theta}(n) d n d h \quad\left(n=\left(\begin{array}{cc}
I & X \\
0 & I
\end{array}\right), X=\left(\begin{array}{cc}
x & y \\
z & x
\end{array}\right)\right)
$$


is absolutely convergent and equal to the (finite) sum $\sum_{\lambda \in F^{x}} \Psi\left(\lambda, f^{\theta}\right)+\sum_{i= \pm} \Psi^{i}\left(f^{\theta}\right)$, where $\Psi\left(\lambda, f^{\theta}\right)=\prod_{v} \Psi\left(\lambda, f_{v}^{\theta}\right), \Psi^{i}\left(f^{\theta}\right)=\prod_{v} \Psi^{i}\left(f_{v}^{\theta}\right)$. Here, if $u=\lambda^{-1}\left(1-y z-\theta^{-1} x^{2}\right)$, we put

$$
\Psi\left(\lambda, f_{v}^{\theta}\right)=|\theta|_{v}^{-2}|\lambda|_{v}^{-3} \int_{F_{v}^{3}} f_{v}^{\theta}\left(\left(\begin{array}{cccc}
0 & u & y & x \\
-u & 0 & -x & \theta z \\
-y & x & 0 & -\theta \lambda \\
-x & -\theta z & \theta \lambda & 0
\end{array}\right)\right) \overline{\boldsymbol{\psi}}_{v}\left(-\lambda^{-1}(y+z)\right) d x d y d z
$$

and

$$
\Psi^{i}\left(f_{v}^{\theta}\right)=\int_{F_{v}} f_{v}^{\theta}\left(i\left(\begin{array}{cccc}
0 & u & 1 & 0 \\
-u & 0 & 0 & \theta \\
-1 & 0 & 0 & 0 \\
0 & -\theta & 0 & 0
\end{array}\right)\right) \overline{\boldsymbol{\psi}}_{v}(u) d u .
$$

Similarly, introduce $\tilde{f}=\otimes \tilde{f}_{v}$ on $\mathbf{X}(1)(\mathbb{A})$ by $\tilde{f}_{v}\left(g \Theta g^{-1} J\right)=\int_{C_{v} / Z_{v}} f_{v}(g h) d h$. Then

$$
\int_{N \backslash \mathbf{N}(\mathbb{A})} \int_{\mathbf{Z}(\mathbb{A}) C \backslash \mathbf{C}(\mathbb{A})} \sum_{\gamma \in Z \backslash G} f\left(n^{-1} \gamma h\right) \bar{\psi}_{0}(n) d n d h
$$

is absolutely convergent and equal to the (finite) sum $\sum_{\lambda \in F^{\times}} \Psi(\lambda, \tilde{f})+\sum_{i= \pm} \Psi^{i}(\tilde{f})$, where $\Psi(\lambda, \tilde{f})=\prod_{v} \Psi\left(\lambda, \tilde{f}_{v}\right), \Psi^{i}(\tilde{f})=\prod_{v} \Psi^{i}\left(\tilde{f}_{v}\right)$. Here, with $u=-\lambda^{-1}\left(1-y z-x^{2}\right)$, we put

$$
\Psi\left(\lambda, \tilde{f}_{v}\right)=|\lambda|_{v}^{-3} \int_{F_{v}^{3}} \tilde{f}_{v}\left(\left(\begin{array}{cccc}
0 & u & y & x \\
-u & 0 & -x & z \\
-y & x & 0 & \lambda \\
-x & -z & -\lambda & 0
\end{array}\right)\right) \bar{\psi}_{v}(2 x / \lambda) d x d y d z
$$

and

$$
\Psi^{i}\left(\tilde{f}_{v}\right)=\int_{F_{v}} \tilde{f}\left(i\left(\begin{array}{rrrr}
0 & u & 0 & 1 \\
-u & 0 & -1 & 0 \\
0 & 1 & 0 & 0 \\
-1 & 0 & 0 & 0
\end{array}\right)\right) \bar{\psi}_{v}(u) d u .
$$

Proof. The integral (2.1) is a sum of two parts, according to Proposition 1(c). The main part is

$$
\begin{aligned}
& \int_{N \backslash \mathbf{N}(\mathbb{A})} \int_{\mathbf{Z}(\mathbb{A}) C_{\theta} \backslash \mathbf{C}_{\theta}(\mathbb{A})} \sum_{\gamma \in B \gamma_{0} C_{\theta}} f(n \gamma h) \bar{\psi}_{\theta}(n) d n d h \\
= & \sum_{\lambda \in F^{\times}} \int_{\mathbf{C}_{\theta}(\mathbb{A}) / \mathbf{Z}(\mathbb{A})} f\left(n\left(\begin{array}{ll}
I & 0 \\
0 & \lambda
\end{array}\right) \gamma_{0} h\right) \overline{\boldsymbol{\psi}}(z-\theta y) d n d h,
\end{aligned}
$$

since $B \gamma_{0} C_{\theta}=N \Lambda \gamma_{0} C_{\theta}, \Lambda=\left\{\left(\begin{array}{cc}I & 0 \\ 0 & \lambda\end{array}\right) ; \lambda \in \mathrm{GL}(1)\right\}$. By matrix multiplication and the definition of $f_{v}^{\theta}$ we have that the local factor

$$
\int_{N_{v}} \int_{C_{\theta, v} / Z_{v}} f_{v}\left(n\left(\begin{array}{ll}
I & 0 \\
0 & \lambda
\end{array}\right) \gamma_{0} h\right) \overline{\boldsymbol{\psi}}_{v}(z-\theta y) d n d h
$$


is equal to $\Psi\left(\lambda, f_{v}^{\theta}\right)$, as defined in (2.2). The finiteness of the sum over $\lambda$ is easily proven on considering the map $g \mapsto g \Theta_{\theta} g^{-1} J$, and using the fact that $f$ is compactly supported.

The second part is

$$
\begin{aligned}
& \int_{N \backslash \mathbf{N}(\mathbb{A})} \int_{\mathbf{Z}(\mathbb{A}) C_{\theta} \backslash \mathbf{C}_{\theta}(\mathbb{A})} \sum_{\substack{\nu \in N, m \in M \cap B / \Lambda \\
\eta \in N \cap C_{\theta} \backslash C_{\theta}}} f(n \nu m \eta h) \bar{\psi}_{\theta}(n) d h d n \\
= & \int_{\mathbf{N}(\mathbb{A})} \int_{N \cap C_{\theta} \backslash \mathbf{C}_{\theta}(\mathbb{A})} \sum_{\substack{a \in F^{*} ; b \in F\\
}} f\left(n\left(\begin{array}{cc}
A & 0 \\
0 & \varepsilon A \varepsilon
\end{array}\right) h\right) \bar{\psi}_{\theta}(n) d n d h \quad\left(A=\left(\begin{array}{ll}
a & b \\
0 & 1
\end{array}\right)\right) .
\end{aligned}
$$

Write $h$ in the form $\left(\begin{array}{ll}I & t \\ 0 & I\end{array}\right) h$, where now $h \in \mathbf{N}(\mathbb{A}) \cap \mathbf{C}_{\theta}(\mathbb{A}) \backslash \mathbf{C}_{\theta}(\mathbb{A})$ and $t=\left(\begin{array}{cc}x & y \\ \theta y & x\end{array}\right)(x, y$ range over $\mathbb{A} / F)$. Since

$$
\left(\begin{array}{cc}
A & 0 \\
0 & \varepsilon A \varepsilon
\end{array}\right)\left(\begin{array}{ll}
I & t \\
0 & I
\end{array}\right)\left(\begin{array}{cc}
A^{-1} & 0 \\
0 & \varepsilon A^{-1} \varepsilon
\end{array}\right)=\left(\begin{array}{cc}
I & A t \varepsilon A^{-1} \varepsilon \\
0 & I
\end{array}\right)
$$

and

$$
\operatorname{tr}\left[\varepsilon A^{-1} \varepsilon T A t\right]=a^{-1} \theta y-\theta a y-a^{-1} b^{2} \theta^{2} y-2 \theta b x,
$$

integrating over $x$ in $\mathbb{A} / F$ we obtain 0 unless $b=0$, in which case the volume $|\mathbb{A} / F|=1$ is obtained. Integrating over $y$ in $\mathbb{A} / F$ again we get 0 , unless $a= \pm 1$, in which case the volume $|\mathbb{A} / F|=1$ is obtained. Our integral is then the sum over $i= \pm$ of

$$
\int_{\mathbf{N}(\mathbb{A}) / \mathbf{N}(\mathbb{A}) \cap \mathbf{C}_{\theta}(\mathbb{A})} f^{\theta}\left(i n \Theta_{\theta} n^{-1} J\right) \bar{\psi}_{\theta}(n) d n .
$$

The local factors of this integral are equal to those of (2.3).

The integral (2.4) is similarly handled. By Proposition 1(b) it is expressed as a sum of two parts. Since $P \gamma_{1} C=N \Lambda \gamma_{1} C$, the main part takes the form

$$
\begin{aligned}
& \int_{\mathbf{N}(\mathbb{A}) / N} \int_{\mathbf{Z}(\mathbb{A}) C \backslash \mathbf{C}(\mathbb{A})} \sum_{\substack{\nu \in N, \lambda \in F^{\times} \\
\eta \in C}} f\left(n \nu\left(\begin{array}{ll}
I & 0 \\
0 & \lambda
\end{array}\right) \gamma_{1} \eta h\right) \overline{\boldsymbol{\psi}}(\operatorname{tr} X) d n d h \\
& =\sum_{\lambda \in F^{\times}} \int_{\mathbf{N}(\mathbb{A})} \int_{\mathbf{C}(\mathbb{A}) / \mathbf{Z}(\mathbb{A})} f\left(n\left(\begin{array}{ll}
I & 0 \\
0 & \lambda
\end{array}\right) \gamma_{1} h\right) \bar{\psi}(\operatorname{tr} X) d n d h .
\end{aligned}
$$

Note that

$\gamma_{1} C \gamma_{1}^{-1} \cap P=\left\{\left(\begin{array}{cc}A & 0 \\ 0 & \varepsilon A \varepsilon\end{array}\right)\right\}, \quad$ since $\quad \gamma_{1}\left(\begin{array}{ccc}a & 0 & b \\ 0 & A^{\prime} & 0 \\ c & 0 & d\end{array}\right) \gamma_{1}^{-1}=\left(\begin{array}{cc}A & 0 \\ 0 & \varepsilon A \varepsilon\end{array}\right), A=\left(\begin{array}{ll}a & b \\ c & d\end{array}\right), A^{\prime}=w A w$.

Using the definition of $\tilde{f}_{v}$ we then obtain the sum over $\lambda \in F^{\times}$of $\Psi(\lambda, \tilde{f})=\prod_{v} \Psi\left(\lambda, \tilde{f}_{v}\right)$, where $\Psi\left(\lambda, \tilde{f}_{v}\right)$ is defined by $(2.5)$. 
The second part of (2.4) is

$$
\begin{aligned}
& \int_{\mathbf{N}(\mathbb{A}) / N} \int_{\mathbf{Z}(\mathbb{A}) C \backslash \mathbf{C}(\mathbb{A})} \sum_{\substack{\nu \in N / N \cap C \\
\xi \in H / A, \eta \in C / Z}} f(n \nu d(\xi) \eta h) \overline{\boldsymbol{\psi}}(\operatorname{tr} X) d n d h \\
= & \int_{\mathbf{N}(\mathbb{A}) / N \cap C} \int_{\mathbf{C}(\mathbb{A}) / \mathbf{Z}(\mathbb{A})} \sum_{\xi \in H / A} f(n d(\xi) h) \overline{\boldsymbol{\psi}}(\operatorname{tr} X) d n d h,
\end{aligned}
$$

where $H=\mathrm{GL}(2, F), A=$ diagonal subgroup in $H$, and $d(\xi)=\operatorname{diag}\left(\xi, w^{t} \xi^{-1} w\right)$. Since

$$
d(\xi)\left(\begin{array}{cc}
I & Y \\
0 & I
\end{array}\right) d(\xi)^{-1}=\left(\begin{array}{cc}
I & \xi Y w^{t} \xi w \\
0 & I
\end{array}\right), Y=\left(\begin{array}{cc}
0 & y \\
z & 0
\end{array}\right), \operatorname{tr} \xi Y w^{t} \xi w=2 \alpha \gamma y+2 \beta \delta z \text { if } \xi=\left(\begin{array}{cc}
\alpha & \beta \\
\gamma & \delta
\end{array}\right)
$$

changing $h \mapsto\left(\begin{array}{cc}I & Y \\ 0 & I\end{array}\right) h$, and integrating over $y, z$ in $\mathbb{A} / F$, we obtain 0 unless $\xi$ is $I$ or $w$, modulo the diagonal subgroup $A$. Using now the definition of $\tilde{f}$, we obtain the sum over $i= \pm$ of $\Psi^{i}(\tilde{f})=\prod_{v} \Psi^{i}\left(\tilde{f}_{v}\right)$, where $\Psi^{i}\left(\tilde{f}_{v}\right)$ is defined by (2.6).

4. Asymptotes. To compare the geometric side (2.1) (or (2.4)) of the Fourier summation formula with an analogous expression for a different group, we need to characterize the Fourier orbital integrals (2.2) and (2.3) (or (2.5) and (2.6)) of which the formula consists. To express this characterization, let $F$ be a local field, $\psi: F \rightarrow \mathbb{C}^{\times}$a nontrivial character, $d x$ the auto dual Haar measure on $F, \hat{\varphi}(x)=\int_{F} \varphi(y) \psi(x y) d y$ the Fourier transform of $\varphi \in C_{c}^{\infty}(F)$, and $\gamma_{\psi}(a)\left(a \in F^{\times}\right)$the Weil function, defined by

$$
\int_{F} \varphi(x) \psi\left(\frac{1}{2} a x^{2}\right) d x=\gamma_{\psi}(a)|a|^{-1 / 2} \int_{F} \hat{\varphi}(x) \psi\left(-\frac{1}{2} a^{-1} x^{2}\right) d x .
$$

Then $\gamma(a)=\gamma_{\psi}(1) \gamma_{\psi}(-a)$ is a function from $F^{\times} / F^{\times 2}$ to the group of the complex fourth roots of unity, satisfying $\gamma(a) \gamma(b)=\gamma(a b)(a, b)$ (see [We]), where $(a, b)$ is the Hilbert symbol $\left(=1\right.$ if $a x^{2}-b y^{2},=-1$ if not). Put $G=\operatorname{GSp}(4, F) ;|\cdot|$ is the normalized absolute value on $F$. We shall later use the results of this section with $\psi$ replaced by $\bar{\psi}$.

The complex valued functions $\Psi_{1}, \Psi_{2}$ on $F$ are called equivalent, and we write $\Psi_{1} \equiv \Psi_{2}$, if $\Psi_{1}(\lambda)=\Psi_{2}(\lambda)$ for $\lambda$ in some neighborhood of 0 ( $F$ non archimedean), and if $\Psi_{1}, \Psi_{2}$ have equal derivatives of all orders at $\lambda=0$ ( $F$ archimedean $)$.

Proposition 3. (a) For every function $f \in C_{c}^{\infty}(G / Z)$ and $\theta \in F^{\times}$, the function $\Psi\left(\lambda, f^{\theta}\right)$ of (2.2) is compactly supported in $\lambda$ on $F$, smooth (locally constant if $F$ is nonarchimedean) on $F^{\times}$, and

$$
\begin{aligned}
& \Psi\left(\lambda, f^{\theta}\right) \equiv \gamma_{\psi}(-1) \gamma_{\psi}(\theta)|2 \lambda|^{-1}|\theta|^{-3 / 2} \\
& \cdot\left\{(\theta, 2 \lambda) \psi(-2 / \lambda) \Psi^{+}\left(\lambda, f^{\theta}\right)+(\theta,-2 \lambda) \psi(2 / \lambda) \Psi^{-}\left(\lambda, f^{\theta}\right)\right\},
\end{aligned}
$$

where $\Psi^{i}\left(\lambda, f^{\theta}\right)$ are smooth functions in a neighborhood of $\lambda=0$ whose values at $\lambda=0$ are the $\Psi^{i}\left(f^{\theta}\right)$ which are defined by $(2.3), i= \pm$. 
Conversely, if $\Psi(\lambda)$ is a complex valued compactly supported function in $\lambda$ on $F$, smooth on $F^{\times}$, and

$$
\Psi(\lambda) \equiv \gamma_{\psi}(-1) \gamma_{\psi}(\theta)|2 \lambda|^{-1}|\theta|^{-3 / 2}\left\{(\theta, 2 \lambda) \psi(-2 / \lambda) \Psi^{+}(\lambda)+(\theta,-2 \lambda) \psi(2 / \lambda) \Psi^{-}(\lambda)\right\},
$$

for some smooth complex valued functions $\Psi^{+}(\lambda), \Psi^{-}(\lambda)$, then there exists $f \in C_{c}^{\infty}(G / Z)$ with $\Psi\left(\lambda, f^{\theta}\right)=\Psi(\lambda)$ for all $\lambda \in F^{\times}$, and $\Psi^{i}\left(\lambda, f^{\theta}\right) \equiv \Psi^{i}(\lambda)(i= \pm)$.

(b) For every function $f \in C_{c}^{\infty}(G / Z)$ the function $\Psi(\lambda, \tilde{f})$ of $(2.5)$ is compactly supported in $\lambda$ on $F$, smooth on $F^{\times}$, and

$$
\Psi(\lambda, \tilde{f}) \equiv|2 \lambda|^{-1} \psi(2 / \lambda) \Psi^{+}(\lambda, \tilde{f})+|2 \lambda|^{-1} \psi(-2 / \lambda) \Psi^{-}(\lambda, \tilde{f}),
$$

where $\Psi^{i}(\lambda, \tilde{f})$ are smooth near $\lambda=0$ and whose values at $\lambda=0$ are the $\Psi^{i}(\tilde{f})$ which are defined by (2.6), $i= \pm$.

Conversely, if $\Psi(\lambda)$ is a complex valued compactly supported function in $\lambda$ on $F$, smooth on $F^{\times}$, and

$$
\Psi(\lambda) \equiv|2 \lambda|^{-1} \psi(2 / \lambda) \Psi^{+}(\lambda)+|2 \lambda|^{-1} \psi(-2 / \lambda) \Psi^{-}(\lambda),
$$

for some smooth complex valued functions $\Psi^{+}(\lambda), \Psi^{-}(\lambda)$, then there exists $f \in C_{c}^{\infty}(G / Z)$ with $\Psi(\lambda, \tilde{f})=\Psi(\lambda)$ for all $\lambda \in F^{\times}$, and $\Psi^{i}(\lambda, \tilde{f}) \equiv \Psi^{i}(\lambda)(i= \pm)$.

Proof. (a) Consider the case of $\theta \in F-F^{2}$. The function $f^{\theta}$ is smooth with compact support on the quadric $b_{1} b_{2}+b^{2}-a d=\theta$ (in $F^{5}$ ) part of $X(\theta)$ (see Proposition 1(d)). This quadric is the union of the open subsets $\left\{b_{1} \neq 0\right\}$ and $\{d \neq 0\}$, since $\theta$ is not a square in $F$. If $f^{\theta}$ is supported on $\{d \neq 0\}$, the integral $\Psi\left(\lambda, f^{\theta}\right)$ of $(2.2)$ is zero for $\lambda$ in some neighborhood of zero. Assume then that $f^{\theta}$ is supported on $\left\{b_{1} \neq 0\right\}$. This set is parametrized by $b_{1}, b, a, d$. The substitution $z \mapsto u=\lambda^{-1}\left(1-y z-\theta^{-1} x^{2}\right), d z=|\lambda / y| d u$, gives

$$
\Psi\left(\lambda, f^{\theta}\right)=|\theta \lambda|^{-2} \iiint \varphi(u, x, y, \lambda) \psi\left(-\lambda^{-1}\left(y+y^{-1}\right)+u / y+(\theta \lambda y)^{-1} x^{2}\right) d u d x d^{\times} y,
$$

where

$$
\varphi(u, x, y, \lambda)=f^{\theta}\left(\left(\begin{array}{cccc}
0 & u & y & x \\
-u & 0 & -x & \left(\theta-x^{2}-\theta \lambda u\right) / y \\
* & * & 0 & -\theta \lambda \\
* & * & \theta \lambda & 0
\end{array}\right)\right)
$$

is a smooth compactly supported function on the subset $\{y \neq 0\}$ of $F^{4}$. To simplify the notations, assume that $\varphi(u, x, y, \lambda)$ is a product $\varphi_{1}(u) \varphi_{2}(x) \varphi_{3}(y) \varphi_{4}(\lambda)$, where $\varphi_{i} \in$ $C_{c}^{\infty}(F)(i=1,2,4)$ and $\varphi_{3} \in C_{c}^{\infty}\left(F^{\times}\right)$. We then get that $\Psi\left(\lambda, f^{\theta}\right)$ is

$$
\begin{aligned}
& =|\theta \lambda|^{-2} \varphi_{4}(\lambda) \int_{F^{\times}} \hat{\varphi}_{1}\left(y^{-1}\right) \varphi_{3}(y)\left\{\int_{F} \varphi_{2}(x) \psi\left((\theta \lambda y)^{-1} x^{2}\right) d x\right\} \psi\left(-\lambda^{-1}\left(y+y^{-1}\right)\right) d^{\times} y \\
& =|\theta \lambda|^{-2} \varphi_{4}(\lambda) \int_{F^{\times}} \hat{\varphi}_{1}\left(y^{-1}\right) \varphi_{3}(y) \gamma_{\psi}(2 \theta \lambda y)|\theta \lambda y / 2|^{1 / 2} \\
& \cdot\left\{\int_{F} \hat{\varphi}_{2}(x) \psi\left(-\frac{1}{4} \theta \lambda y x^{2}\right) d x\right\} \psi\left(-\lambda^{-1}\left(y+y^{-1}\right)\right) d^{\times} y .
\end{aligned}
$$


Assume now that $F$ is nonarchimedean. For $\lambda$ near $0, \Psi\left(\lambda, f^{\theta}\right)$ equals

$$
|2|^{-1 / 2}|\theta \lambda|^{-3 / 2} \varphi_{2}(0) \varphi_{4}(0) \int_{F^{\times}} \hat{\varphi}_{1}\left(y^{-1}\right) \varphi_{3}(y)|y|^{1 / 2} \gamma_{\psi}(2 \theta \lambda y) \psi\left(-\lambda^{-1}\left(y+y^{-1}\right)\right) d^{\times} y .
$$

We shall prove below the following

Lemma 3.1. For any $\varphi$ in $C_{c}^{\infty}\left(F^{\times}\right)$, the value of $\int_{F^{\times}} \varphi(y) \psi\left(\lambda^{-1}\left(y+y^{-1}\right)\right) d^{\times} y$ at $\lambda$ near zero is

$$
\gamma_{\psi}(2 \lambda)|\lambda / 2|^{1 / 2} \psi(2 / \lambda) \varphi(1)+\gamma_{\psi}(-2 \lambda)|\lambda / 2|^{1 / 2} \psi(-2 / \lambda) \varphi(-1) .
$$

Hence for $\lambda$ near zero we obtain

$$
\begin{aligned}
& \Psi\left(\lambda, f^{\theta}\right)=|2|^{-1}|\theta|^{-3 / 2}|\lambda|^{-1} \varphi_{2}(0) \varphi_{4}(0) \\
& \cdot\left\{\gamma_{\psi}(-2 \lambda) \psi(-2 / \lambda) \hat{\varphi}_{1}(1) \varphi_{3}(1) \gamma_{\psi}(2 \theta \lambda)+\gamma_{\psi}(2 \lambda) \psi(2 / \lambda) \hat{\varphi}_{1}(-1) \varphi_{3}(-1) \gamma_{\psi}(-2 \theta \lambda)\right\} .
\end{aligned}
$$

Since $\gamma_{\psi}(-2 \lambda) \gamma_{\psi}(2 \theta \lambda)=\gamma_{\psi}(-1) \gamma_{\psi}(\theta)(2 \lambda, \theta)$, we obtain

$$
\begin{aligned}
& =\gamma_{\psi}(-1) \gamma_{\psi}(\theta)|2|^{-1}|\theta|^{-3 / 2}|\lambda|^{-1} \varphi_{2}(0) \varphi_{4}(0)\left\{\hat{\varphi}_{1}(1) \varphi_{3}(1)(\theta, 2 \lambda) \psi(-2 / \lambda)\right. \\
& \left.+\hat{\varphi}_{1}(-1) \varphi_{3}(-1)(\theta,-2 \lambda) \psi(2 / \lambda)\right\} \\
& =\gamma_{\psi}(-1) \gamma_{\psi}(\theta)|2|^{-1}|\theta|^{-3 / 2}|\lambda|^{-1}\left\{(\theta, 2 \lambda) \psi(-2 / \lambda) \int_{F} \varphi(u, 0,1,0) \psi(u) d u\right. \\
& \left.+(\theta,-2 \lambda) \psi(2 / \lambda) \int_{F} \varphi(-u, 0,-1,0) \psi(u) d u\right\} .
\end{aligned}
$$

This is the asserted asymptotic behavior.

Assume next that $F=\mathbb{R}$, the field of real numbers. Put $\varphi_{5}(y)=\hat{\varphi}_{1}\left(y^{-1}\right) \varphi_{3}(y)|y|^{1 / 2}$, and $\varphi_{2}^{*}(t)=\int_{\mathbb{R}} \hat{\varphi}_{2}(x) \psi\left(\frac{1}{2} t x^{2}\right) d x$. Then

$$
\begin{aligned}
\Psi\left(\lambda, f^{\theta}\right) & =2^{-1 / 2}|\theta \lambda|^{-3 / 2} \varphi_{4}(\lambda)\left\{\gamma_{\psi}(2 \theta \lambda) \int_{\mathbb{R}_{+}^{\times}} \varphi_{5}(y) \varphi_{2}^{*}\left(-\frac{1}{2} \theta \lambda y\right) \psi\left(-\lambda^{-1}\left(y+y^{-1}\right)\right) d^{\times} y\right. \\
& \left.+\gamma_{\psi}(-2 \theta \lambda) \int_{\mathbb{R}_{+}^{\times}} \varphi_{5}(-y) \varphi_{2}^{*}\left(\frac{1}{2} \theta \lambda y\right) \psi\left(-\lambda^{-1}\left(y+y^{-1}\right)\right) d^{\times} y\right\} .
\end{aligned}
$$

For $h \in C_{c}^{\infty}\left(\mathbb{R}_{+}^{\times}\right)$we have

$$
\int_{\mathbb{R}_{+}^{\times}} h(y) \psi\left(\frac{y+y^{-1}}{2 \lambda}\right) d^{\times} y=2 \psi\left(\lambda^{-1}\right) \int_{\mathbb{R}_{+}^{\times}} h\left(x^{2}\right) \psi\left(\frac{\left(x-x^{-1}\right)^{2}}{2 \lambda}\right) d^{\times} x
$$

$\left(y=x^{2}\right)$. On changing $x-x^{-1}=2 u, x=u+\sqrt{u^{2}+1}$, this becomes

$$
=2 \psi\left(\lambda^{-1}\right) \int_{\mathbb{R}} h\left(\left(u+\sqrt{u^{2}+1}\right)^{2}\right) \psi\left(2 u^{2} / \lambda\right) \frac{d u}{\sqrt{u^{2}+1}}
$$




$$
=\gamma_{\psi}(\lambda)|\lambda|^{1 / 2} \psi\left(\lambda^{-1}\right) \int_{\mathbb{R}} \hat{g}(u) \psi\left(-\frac{\lambda}{8} u^{2}\right) d u,
$$

where $g(u)=h\left(\left(u+\sqrt{u^{2}+1}\right)^{2}\right) / \sqrt{u^{2}+1}$. To use this formula in our context, put $g_{i}(\lambda)$

$$
=\int_{\mathbb{R}} \psi\left(i \frac{\lambda}{16} v^{2}\right) d v \int_{\mathbb{R}}\left(u^{2}+1\right)^{-1 / 2} \varphi_{5}\left(i\left(u+\sqrt{u^{2}+1}\right)^{2}\right) \varphi_{2}^{*}\left(-\frac{i}{2} \theta \lambda\left(u+\sqrt{u^{2}+1}\right)^{2}\right) \psi(u v) d u
$$

for $i= \pm$. Note that $g_{i}$ are smooth functions on $\mathbb{R}$ (although $\varphi_{2}^{*}$ is not a Schwartz function), and that $g_{i}(0)=\varphi_{2}(0) \varphi_{5}(i 1)$. Then $\Psi\left(\lambda, f^{\theta}\right)$

$$
=\gamma_{\psi}(-1) \gamma_{\psi}(\theta) 2^{-1}|\theta|^{-3 / 2}|\lambda|^{-1} \varphi_{4}(\lambda)\left\{(2 \lambda, \theta) \psi(-2 / \lambda) g_{+}(\lambda)+(-2 \lambda, \theta) \psi(2 / \lambda) g_{-}(\lambda)\right\},
$$

and the asymptotic behavior asserted in (a) of the proposition follows.

The case of $\theta \in F^{\times 2}$ is similar to that of (b), which is done next.

(b) Consider the integral $\Psi(\lambda, \tilde{f})$ of (2.5). The function $\tilde{f}$ is smooth and compactly supported on the quadric $X=\left\{b_{1} b_{2}+b^{2}-a d=1\right.$ in $\left.F^{5}\right\}$ described by Proposition 1(d). This $X$ is the union of four open subsets: $V_{i}=\left\{b+\frac{i}{2}\left(b_{1}-b_{2}\right) \neq 0\right\}(i= \pm), V_{b_{1}}=\left\{b_{1} \neq 0\right\}, V_{d}=$ $\{d \neq 0\}$. If $\tilde{f}$ is supported on $\{d \neq 0\}$ then $\Psi(\lambda, \tilde{f})$ is zero for $\lambda$ in some neighborhood of zero. If $\tilde{f}$ is supported on $\left\{b_{1} \neq 0\right\}$, writing $z=\left(1+\lambda u-x^{2}\right) / y$ we see that $\Psi(\lambda, \tilde{f})$ is rapidly decreasing as $\lambda \rightarrow 0$. Assume then that $\tilde{f}$ is supported on $V_{+}$. Change variables: $x \mapsto \frac{1}{2}(y+z), y \mapsto x+\frac{1}{2}(y-z), z \mapsto x-\frac{1}{2}(y-z)$, and note that $x^{2}+y z$, hence $u$, are not changed. Then we get

$$
\begin{gathered}
\Psi(\lambda, \tilde{f})=|\lambda|^{-3} \iiint_{F^{3}} \tilde{f}\left(\left(\begin{array}{cccc}
0 & u & x+\frac{1}{2}(y-z) & \frac{1}{2}(y+z) \\
-u & 0 & -\frac{1}{2}(y+z) & x-\frac{1}{2}(y-z) \\
* & * & 0 & \lambda \\
* & * & -\lambda & 0
\end{array}\right)\right) \psi\left(\lambda^{-1}(y+z)\right) d x d y d z, \\
\Psi(-\lambda, \tilde{f})=|\lambda|^{-2} \iint_{F^{2}} \int_{F^{\times}} \varphi(u, x, y, \lambda) \psi\left(-\lambda^{-1}\left(y+y^{-1}\right)\right) \psi(u / y) \psi\left(\lambda^{-1} y^{-1} x^{2}\right) d u d x d^{\times} y,
\end{gathered}
$$

where

$$
\varphi(u, x, y, \lambda)=\tilde{f}\left(\left(\begin{array}{cccc}
0 & u & * & * \\
-u & 0 & * & * \\
* & * & 0 & -\lambda \\
* & * & \lambda & 0
\end{array}\right)\right)
$$

is smooth with compact support on the subset $\{y \neq 0\}$ of $F^{4}$. We obtained precisely the same integral as in the nonsplit case (a), where $\theta \in F-F^{2}$, but with $\theta=1$. The computation there applies with $\theta=1$ too. The leading term in the asymptotic expansion of $\Psi(\lambda, \tilde{f})$ is then

$$
\begin{aligned}
& |2 \lambda|^{-1}\left\{\psi(2 / \lambda) \int_{F} \varphi(u, 0,1,0) \psi(u) d u+\psi(-2 / \lambda) \int_{F} \varphi(-u, 0,-1,0) \psi(u) d u\right\} \\
& =|2 \lambda|^{-1} \psi(2 / \lambda) \Psi^{+}(\lambda, \tilde{f})+|2 \lambda|^{-1} \psi(-2 / \lambda) \Psi^{-}(\lambda, \tilde{f}) .
\end{aligned}
$$


The treatment of $\tilde{f}$ which is supported on $V_{-}$is similarly carried out. A general $\tilde{f}$ can be expressed as $f_{+}+f_{-}+f_{b_{1}}+f_{d}$, with $f_{*}$ supported on the open set $V_{*}$. The case where $F$ is the field of complex numbers is similarly handled.

For the opposite direction(s), given $\Psi(\lambda)$ choose $f_{1}$ with $\Psi^{i}\left(\lambda, f_{1}^{\theta}\right)$ of $(2.3)$ (or $\Psi^{i}\left(\lambda, \tilde{f}_{1}\right)$ of $(2.6)$ ) equivalent to $\Psi^{i}(\lambda)$. Then $\Psi(\lambda)-\Psi\left(\lambda, f_{1}^{\theta}\right)$ (or $\Psi(\lambda)-\Psi\left(\lambda, \tilde{f}_{1}\right)$ ) is smooth and compactly supported on $F^{\times}$, and it is easy to find $f_{2}$ with $\Psi\left(\lambda, f_{2}^{\theta}\right)$ (or $\Psi\left(\lambda, \tilde{f}_{2}\right)$ ) equal to this function on $F^{\times}$. Then $f=f_{1}+f_{2}$ is the required function.

It remains to prove Lemma 3.1. It follows on taking $a=b= \pm 2 / \lambda$ in the following Lemma. Thus let $F$ be a nonarchimedean local field, $R$ its ring of integers, $\pi$ a generator of its maximal ideal $(\boldsymbol{\pi})$, and $v$ the valuation on $F^{\times}$, normalized by $v(\boldsymbol{\pi})=1$.

Lemma 3.2. Given a nontrivial character $\psi$ of $F$, and $N \geq 2 v(2)+1$, there is a constant A such that for any $a, b \in F^{\times}$with $v(b) \leq A$ we have that $\int_{1+\left(\pi^{N}\right)} \psi\left(\frac{1}{2}\left(a t+b t^{-1}\right)\right) d t$ is equal to $\psi(a c)|a|^{-1 / 2} \gamma_{\psi}(a c)$ if $b / a=c^{2}, c \equiv 1 \bmod \left(\boldsymbol{\pi}^{N}\right)$, and to zero otherwise.

Proof. It suffices to prove this for $\psi$ such that $R^{\perp}=\{x \in F ; \psi(x y)=1$ for all $y \in R\}$ is $R$. In this case one may take $A=-2 N-v(2)$. The integral of our lemma is equal to

$$
\sum_{t \in 1+\left(\boldsymbol{\pi}^{N}\right) / 1+\left(\boldsymbol{\pi}^{n}\right)} \int_{1+\left(\boldsymbol{\pi}^{n}\right)} \psi\left(\frac{1}{2}\left(a t u+b t^{-1} u^{-1}\right)\right) d u, \quad n=\left[\frac{1}{2}(1+v(2)-v(b))\right] .
$$

If $t \in 1+\left(\boldsymbol{\pi}^{N}\right)$ is such that the integral over $1+\left(\boldsymbol{\pi}^{n}\right)$ is nonzero, then on writing $u=$ $1+\pi^{n} x,|x| \leq 1$, we see that $v\left(a t-b t^{-1}\right)+n-v(2) \geq 0$. Hence $v\left(b^{-1} a t^{2}-1\right) \geq N+v(2)$, namely $b^{-1} a \equiv 1 \bmod \left(2 \pi^{N}\right)$, and so $a^{-1} b=c^{2}$ with $c \equiv 1 \bmod \left(\pi^{N}\right)$. If $a^{-1} b=c^{2}$ and $c \equiv 1 \bmod \left(\boldsymbol{\pi}^{N}\right)$, then

$$
\int_{1+\left(\boldsymbol{\pi}^{n}\right)} \psi\left(\frac{1}{2}\left(a t+b t^{-1}\right)\right) d t=\int_{1+\left(\pi^{N}\right)} \psi\left(\frac{1}{2} a c\left(t+t^{-1}\right)\right) d t .
$$

If now $\lambda \in F^{\times}$has $v(\lambda) \geq 2 N+v(2)$, then

$$
\begin{aligned}
& \int_{1+\left(\boldsymbol{\pi}^{N}\right)} \psi\left(\frac{t+t^{-1}}{2 \lambda}\right) d t=|2| \psi\left(\lambda^{-1}\right) \int_{1+\left(\frac{1}{2} \boldsymbol{\pi}^{N}\right)} \psi\left(\frac{\left(x-x^{-1}\right)^{2}}{2 \lambda}\right) d x \quad\left(t=x^{2}\right) \\
& =|2| \psi\left(\lambda^{-1}\right) \int_{\left(\frac{1}{2} \boldsymbol{\pi}^{N}\right)} \psi\left(2 y^{2} / \lambda\right) d y=\psi\left(\lambda^{-1}\right)|\lambda|^{1 / 2} \gamma_{\psi}(\lambda),
\end{aligned}
$$

where $x-x^{-1}=2 y$, namely $x=y+\sqrt{1+y^{2}}$. The lemma follows, as does Lemma 3.1.

5. Matching. The geometric side of the Fourier summation formula on $\mathbf{G}=\mathrm{GSp}(4)$ is analogous - and will be compared to - a Fourier summation formula on $\mathbf{H}=\mathrm{GSp}(2)=$ $\mathrm{GL}(2)$. Let $F$ be a global field, and $f^{\prime}=\otimes f_{v}^{\prime}$ a test function on $\mathbf{H}(\mathbb{A})$. Thus $f_{v}^{\prime} \in$ $C_{c}^{\infty}\left(H_{v} / Z_{v}\right)$ for all $v$, and for almost all $v$ this $f_{v}^{\prime}$ is the unit element $f_{v}^{\prime 0}$ in the convolution algebra of $K^{\prime}=H_{v}\left(R_{v}\right)$-biinvariant function on $H_{v} / Z_{v}$. Here $\mathbf{Z}$ denotes (also) 
the center of $\mathbf{H}$. The convolution operator $\left(r\left(f^{\prime}\right) \phi\right)(x)=\int_{\mathbf{H}(\mathbb{A}) / \mathbf{Z}(\mathbb{A})} f^{\prime}(y) \phi(x y) d y$ on $L^{2}(\mathbf{Z}(\mathbb{A}) H \backslash \mathbf{H}(\mathbb{A}))$ is an integral operator $\left(=\int_{\mathbf{Z}(\mathbb{A}) H \backslash \mathbf{H}(\mathbb{A})} K_{f^{\prime}}(x, y) \phi(y) d y\right)$ with the kernel $K_{f^{\prime}}(x, y)=\sum f^{\prime}\left(x^{-1} \gamma y\right)(\gamma \in Z \backslash H)$. The geometric side of the Fourier summation formula for $\mathbf{H}(\mathbb{A})$ is obtained on integrating this kernel against $\bar{\psi}(x)$ on $x$ over $N^{\prime} \backslash \mathbf{N}(\mathbb{A})^{\prime}, \mathbf{N}^{\prime}=$ $\left\{x=\left(\begin{array}{ll}1 & * \\ 0 & 1\end{array}\right)\right\}, \psi(x)=\boldsymbol{\psi}(*)$ if $* \in \mathbb{A} / F$, and $\boldsymbol{\psi}$ is our fixed nontrivial character of $\mathbb{A} / F$, and against $\chi(a)$ over $y=\left(\begin{array}{ll}a & 0 \\ 0 & 1\end{array}\right), a \in \mathbb{A}^{\times} / F^{\times}$, where $\chi$ is a character of $\mathbb{A}^{\times} / F^{\times}$whose square is 1 . Put $\psi=\psi$, and $w=\left(\begin{array}{cc}0 & -1 \\ 1 & 0\end{array}\right)$.

Proposition 4. For any $f^{\prime}=\otimes f_{v}^{\prime}$ on $\mathbf{H}(\mathbb{A})$ and a character $\chi$ of $\mathbb{A}^{\times} / F^{\times}$with $\chi^{2}=1$, the integral

$$
\int_{\mathbb{A} / F} \int_{\mathbb{A}^{\times} / F^{\times}} \sum_{\gamma \in Z \backslash H} f^{\prime}\left(\left(\begin{array}{ll}
1 & x \\
0 & 1
\end{array}\right) \gamma\left(\begin{array}{ll}
a & 0 \\
0 & 1
\end{array}\right)\right) \psi(x) \chi(a) d x d^{\times} a
$$

is equal to the sum of $\sum_{\lambda \in F^{\times}} \Psi_{\chi}\left(\lambda, f^{\prime}\right), \Psi_{\chi}^{+}\left(f^{\prime}\right)$ and $\Psi_{\chi}^{-}\left(f^{\prime}\right)$, where $\Psi_{\chi}\left(f^{\prime}\right)=\prod_{v} \Psi_{\chi}\left(f_{v}^{\prime}\right)$ and

$$
\begin{aligned}
\Psi_{\chi_{v}}\left(\lambda, f_{v}^{\prime}\right) & =\int_{F_{v}} \int_{F_{v}^{\times}} f_{v}^{\prime}\left(\left(\begin{array}{ll}
1 & x \\
0 & 1
\end{array}\right) w\left(\begin{array}{ll}
1 & \lambda \\
0 & 1
\end{array}\right)\left(\begin{array}{ll}
a & 0 \\
0 & 1
\end{array}\right)\right) \boldsymbol{\psi}_{v}(x) \chi_{v}(a) d x d^{\times} a, \\
\Psi_{\chi_{v}}^{-}\left(f_{v}^{\prime}\right) & =\int_{F_{v}} \int_{F_{v}^{\times}} f_{v}^{\prime}\left(\left(\begin{array}{ll}
1 & x \\
0 & 1
\end{array}\right)\left(\begin{array}{ll}
a & 0 \\
0 & 1
\end{array}\right)\right) \psi_{v}(x) \chi_{v}(a) d x d^{\times} a, \quad \Psi_{\chi_{v}}^{+}\left(f_{v}^{\prime}\right)=\Psi_{\chi_{v}}\left(0, f_{v}^{\prime}\right) .
\end{aligned}
$$

This follows at once from the Bruhat decomposition $\mathbf{H}=\mathbf{B}^{\prime} \cup \mathbf{N}^{\prime} w \mathbf{B}^{\prime}$. From

$$
\left(\begin{array}{ll}
1 & x \\
0 & 1
\end{array}\right)\left(\begin{array}{ll}
0 & 1 \\
1 & 0
\end{array}\right)\left(\begin{array}{ll}
1 & \lambda \\
0 & 1
\end{array}\right)\left(\begin{array}{cc}
1 & 0 \\
0 & -1
\end{array}\right)\left(\begin{array}{cc}
1 & -\lambda \\
0 & 1
\end{array}\right)\left(\begin{array}{ll}
0 & 1 \\
1 & 0
\end{array}\right)\left(\begin{array}{cc}
1 & -x \\
0 & 1
\end{array}\right)\left(\begin{array}{cc}
0 & -1 \\
1 & 0
\end{array}\right)=\left(\begin{array}{cc}
2 x(1+\lambda) & 1+2 x \lambda \\
1+2 x \lambda & 2 \lambda
\end{array}\right)
$$

it transpires that the sum over $\lambda$ ranges over a finite set, depending on the support of $f^{\prime}$, that $x$ ranges (in $\Psi_{\chi_{v}}\left(f_{v}^{\prime}\right)$ ) over a compact set in $F_{v}$, and that $a$ ranges there over a compact set in $F_{v}^{\times}$, again depending on the support of $f^{\prime}$.

As in the case of $\mathbf{G}$, we shall characterize next the Fourier orbital integrals $\Psi_{\chi_{v}}\left(\lambda, f_{v}^{\prime}\right)$, as in Jacquet [J2], Proposition 4.2, p. 129. Let $F$ be a local field, and $\chi: F^{\times} \rightarrow\{ \pm 1\}$ a character with $\chi^{2}=1$.

Proposition 5. (a) For every function $f^{\prime} \in C_{c}^{\infty}(H / Z)$ there are smooth compactly supported functions $\Psi_{\chi}^{+}\left(\lambda, f^{\prime}\right)$ and $\Psi_{\chi}^{-}\left(\lambda, f^{\prime}\right)$ on $F$ with $\Psi_{\chi}^{i}\left(0, f^{\prime}\right)=\Psi_{\chi}^{i}\left(f^{\prime}\right)(i= \pm)$ such that $\Psi_{\chi}\left(\lambda, f^{\prime}\right)=\Psi_{\chi}^{+}\left(\lambda, f^{\prime}\right)+\psi\left(\lambda^{-1}\right) \Psi_{\chi}^{-}\left(\lambda, f^{\prime}\right)$ for all $\lambda$ in $F^{\times}$.

(b) For any smooth compactly supported functions $\Psi^{+}(\lambda)$ and $\Psi^{-}(\lambda)$ on $F$ there is a function $f^{\prime}=f_{\chi}^{\prime} \in C_{c}^{\infty}(H / Z)$ with $\Psi_{\chi}\left(\lambda, f^{\prime}\right)=\Psi^{+}(\lambda)+\psi\left(\lambda^{-1}\right) \Psi^{-}(\lambda)$ on $F$.

Proof. Write $\Psi$ for $\Psi_{\chi}$ in the course of the proof. Expressing $H$ as the union of the two open sets $N w N A$ and $\bar{N} N A\left(\bar{N}=w N w^{-1}\right), N=\left\{\left(\begin{array}{ll}1 & * \\ 0 & 1\end{array}\right)\right\}, A=\left\{\left(\begin{array}{ll}* & 0 \\ 0 & *\end{array}\right)\right\}$, we may assume that $f^{\prime}$ is supported on one of these sets. If $f$ is supported on $N w N A$, then $\Psi\left(\lambda, f^{\prime}\right)$ is 
smooth and compactly supported on $F$, and $\Psi\left(0, f^{\prime}\right)=\Psi^{+}\left(f^{\prime}\right)$. Any smooth compactly supported function on $F$ is of the form $\Psi\left(\lambda, f^{\prime}\right)$ for some $f^{\prime} \in C_{c}^{\infty}(H / Z)$ supported on $N w N A$.

Suppose that $f^{\prime}$ is supported on $\bar{N} N A$. Put

$$
T(u, v)=\int f^{\prime}\left(\left(\begin{array}{ll}
1 & 0 \\
v & 1
\end{array}\right)\left(\begin{array}{ll}
1 & u \\
0 & 1
\end{array}\right)\left(\begin{array}{ll}
a & 0 \\
0 & 1
\end{array}\right)\right) \chi(a) d^{\times} a .
$$

It is smooth and compactly supported on $F \times F$, and any smooth compactly supported function on $F \times F$ is of the form $T(u, v)$ for some $f^{\prime}$ supported on $\bar{N} N A$. Since $f^{\prime}$ is trivial on the center and $\chi^{2}=1$, we have that

$$
\Psi\left(\lambda, f^{\prime}\right)=\int T\left(x(x \lambda-1), x^{-1}\right) \psi(x) d x, \quad \Psi^{-}\left(f^{\prime}\right)=\int T(x, 0) \psi(x) d x .
$$

If $T\left(x(x \lambda-1), x^{-1}\right) \neq 0$ then $|x(x \lambda-1)| \leq c_{1},|x|^{-1} \leq c_{2}$ for some positive constants depending on $f^{\prime}$ (or $\left.T\right)$. Then $c_{2}^{-1} \leq|x| \leq\left(1+c_{1} c_{2}\right)|\lambda|^{-1}$ and $|\lambda| \leq\left(1+c_{1} c_{2}\right) c_{2}$. Hence $\Psi\left(\lambda, f^{\prime}\right)$ is smooth on $F^{\times}$and compactly supported on $F$. If $T$ vanishes unless $v$ lies in a compact of $F^{\times}$, then $\Psi\left(\lambda, f^{\prime}\right)$ is smooth at 0 , and $\Psi^{-}\left(f^{\prime}\right)=0$. Hence we may assume that $T$ is supported on $\left\{(u, v) ;|u| \leq c_{1},|v| \leq c_{2}\right\}$, for some fixed $c_{1}$, and a sufficiently small $c_{2}>0$, say with $c_{1} c_{2}<1$. In particular $\Psi^{+}\left(f^{\prime}\right)\left(=\Psi\left(0, f^{\prime}\right)\right)$ is 0 .

Changing $x$ to $z=x-\lambda^{-1}$ we obtain

$$
\Psi\left(\lambda, f^{\prime}\right)=\psi\left(\lambda^{-1}\right) \int T(z(\lambda z+1), \lambda /(1+\lambda z)) \psi(z) d z
$$

If the integrand is not zero then $c_{2}^{-1}|\lambda| \leq|1+\lambda z| \leq c_{1}|z|^{-1}$, hence $|\lambda z| \leq c_{1} c_{2}<1$, and $|1+\lambda z| \geq 1-c_{1} c_{2}>0$, and finally $|z| \leq c_{1}|1+\lambda z|^{-1} \leq c_{1}\left(1-c_{1} c_{2}\right)^{-1}$. The integrand is then a compactly supported function of $z$ in $F$. If $\lambda$ is near zero, so is $\lambda z$, and $1+\lambda z$ lies in a neighborhood of 1 . The value of the integral over $z$ at $\lambda=0$ is clearly $\Psi^{-}\left(f^{\prime}\right)$, hence (a) follows.

For (b), given smooth compactly supported functions $\Psi^{i}(\lambda)$ on $F(i= \pm)$, there is $f_{2}^{\prime}$ supported on $\bar{N} N A$ with $\Psi^{-}\left(f_{2}^{\prime}\right)=\Psi^{-}(0)$, and there is $f_{1}^{\prime}$ supported on $N w N A$ with $\Psi\left(\lambda, f_{1}^{\prime}\right)$ equals to

$$
\Psi^{+}(\lambda)+\psi\left(\lambda^{-1}\right) \Psi^{-}(\lambda)-\Psi\left(\lambda, f_{2}^{\prime}\right),
$$

since the last expression is smooth and compactly supported on $F$. The required function is $f^{\prime}=f_{1}^{\prime}+f_{2}^{\prime}$.

In fact, when $F$ is archimedean we need to show that $f_{2}^{\prime}$ can be chosen such that all derivatives of $\Psi^{-}\left(\lambda, f_{2}^{\prime}\right)$ and $\Psi^{-}(\lambda)$ are equal at $\lambda=0$, since then $(*)$ is smooth on $F$ and $f_{1}^{\prime}$ can be found. For this purpose let $T_{1}, T_{2}$ be smooth functions on $F$ supported on $|u| \leq c_{1},|v| \leq c_{2}$, with $c_{1} c_{2}<1$, and with $\int T_{1}(z) \psi(z) d z=1$. Then the integrand in

$$
\int T_{1}(z(1+\lambda z)) T_{2}(\lambda /(1+\lambda z)) \psi(z) d z
$$


is supported on $|z| \leq c_{1} /\left(1-c_{1} c_{2}\right)$, and the value of the integral at $\lambda=0$ is $T_{2}(0)$. The value of the derivatives of the integral can be computed in terms of the derivatives of $T_{2}$ at $\lambda=0$, hence $f_{2}^{\prime}$ can be chosen so that $\Psi^{-}\left(\lambda, f_{2}^{\prime}\right)$ and all of its derivatives would take at $\lambda=0$ any prescribed values, as asserted.

Note that $\Psi_{\chi}\left(\lambda, f^{\prime}\right)$ depends on a choice of a quadratic character $\chi$. In matching general test functions, any $\chi$ can be taken in the following definition. But the matching of spherical functions (cf. [J2], Proposition 5.1, p. 132) forces the choice of $\chi=\chi_{\theta}$.

Fix $\theta$ in $F^{\times}$, choose $\chi=\chi_{\theta}$, and fix $\psi \neq 1$. The functions $f \in C_{c}^{\infty}(G / Z)$ and $f^{\prime} \in$ $C_{c}^{\infty}(H / Z)$ are called matching if for all $\lambda \in F^{\times}$we have

$$
\Psi\left(\lambda, f^{\theta}\right)=\gamma_{\psi}(-1) \gamma_{\psi}(\theta)|2 \lambda|^{-1}|\theta|^{-3 / 2}(\theta, 2 \lambda) \psi(-2 / \lambda) \Psi_{\chi}\left(\lambda / 4, f^{\prime}\right) .
$$

Corollary 5.1. For every $f \in C_{c}^{\infty}(G / Z)$ there exists $f^{\prime} \in C_{c}^{\infty}(H / Z)$, and for every $f^{\prime}$ there exists an $f$, such that $f$ and $f^{\prime}$ are matching. If $f$ and $f^{\prime}$ are matching, then $\Psi^{i}\left(f^{\theta}\right)=$ $(\theta, i) \Psi_{\chi}^{i}\left(f^{\prime}\right)(i= \pm)$.

Proof. This follows at once from Propositions 3 and 5.

Proposition 6. Suppose that $F$ is a p-adic field, $p>2, \theta$ is a unit, $\chi$ is unramified and $\chi^{2}=1$, and $\psi$ is 1 on $R$ but not on $\pi^{-1} R$. Then the unit elements $f^{0}$ and $f^{\prime 0}$ are matching.

Proof. If $f=f^{0}$ then $f^{\theta}$ (see Proposition 2) is the characteristic function of the set of points in $X(\theta)$ (see Proposition 1(d)) with integral coordinates on the quartic $b_{1} b_{2}+b^{2}-a d=\theta$. Using (2.3) it is clear that $\Psi^{ \pm}\left(f^{\theta}\right)=1$. From (2.2) it follows that $\Psi\left(\lambda, f^{\theta}\right)$ is 0 if $\lambda \notin R$, it is 1 if $\lambda \in R^{\times}$. Suppose that $|\lambda|<1$. As in the beginning of the proof of Proposition 3(a), we have

$$
\Psi\left(\lambda, f^{\theta}\right)=|\lambda|^{-2} \iiint \psi\left(-\lambda^{-1}\left(y+y^{-1}\right)+u y^{-1}+x^{2}(\theta \lambda y)^{-1}\right) d x d^{\times} y d u .
$$

The integration ranges over $(x, y, u)$ in $R^{3}$, with $\theta-x^{2}-\theta \lambda u$ in $y R$. But the integral of $\psi\left(u y^{-1}\right) d u$ over $R \cap\left[\lambda^{-1}\left(1-\theta^{-1} x^{2}\right)+\lambda^{-1} y R\right]$ is 1 if $y \in R^{\times}$and 0 if $y \in R-R^{\times}$. Hence

$$
\Psi\left(\lambda, f^{\theta}\right)=|\lambda|^{-2} \int_{R} \int_{R^{\times}} \psi\left(-\lambda^{-1}\left(y+y^{-1}\right)+x^{2}(\theta \lambda y)^{-1}\right) d x d^{\times} y .
$$

Using the definition of $\gamma_{\psi}$, recorded before Proposition 3, this is

$$
=|\lambda|^{-3 / 2} \int_{R^{\times}} \psi\left(-\lambda^{-1}\left(y+y^{-1}\right)\right) \gamma_{\psi}(2 \theta \lambda y) d^{\times} y .
$$

If $|\lambda|<q^{-1}=|\boldsymbol{\pi}|$, this can be written as $(y=t u)$

$$
|\lambda|^{-3 / 2} \sum_{t \in R^{\times} /(1+\boldsymbol{\pi} R)} \gamma_{\psi}(2 \theta \lambda t) \int_{1+\boldsymbol{\pi} R} \psi\left(-\lambda^{-1}\left(t u+t^{-1} u^{-1}\right)\right) d u .
$$


Lemma 3.2 , with $N=1$ and $A=-2$, asserts that $\int_{1+\pi R} \psi\left(-\lambda^{-1}\left(t u+t^{-1} u^{-1}\right)\right) d u$ is 0 unless $t \equiv \pm 1 \bmod \pi R$, and it is $|\lambda|^{1 / 2} \psi\left(-2 t \lambda^{-1}\right) \gamma_{\psi}(-2 \lambda t)$ if $t= \pm 1$. Hence

$$
\Psi\left(\lambda, f^{\theta}\right)=|\lambda|^{-1} \sum_{t= \pm 1} \gamma_{\psi}(2 \theta \lambda t) \gamma_{\psi}(-2 \lambda t) \psi(-2 t / \lambda)=|\lambda|^{-1}(\theta, \lambda)(\psi(2 / \lambda)+\psi(-2 / \lambda)),
$$

since $\gamma_{\psi}(2 \theta \lambda t) \gamma_{\psi}(-2 \lambda t)=\gamma_{\psi}(-1) \gamma_{\psi}(\theta)(\theta, 2 \lambda t)=(\theta, \lambda): \gamma_{\psi}$ is 1 on units, and $(u, v)=1$ for units $u, v$.

If $|\lambda|=|\boldsymbol{\pi}|$, since $\gamma_{\psi}(2 \theta \lambda y)=\gamma_{\psi}(2 \lambda)(\theta, \lambda)(\lambda, y)$, we have that $\Psi\left(\lambda, f^{\theta}\right)$ is equal to

$$
\begin{aligned}
& |\lambda|^{-3 / 2}(\theta, \lambda) \gamma_{\psi}(2 \lambda) \int_{R^{\times}}(\lambda, y) \psi\left(-\left(y+y^{-1}\right) / \lambda\right) d^{\times} y \\
= & |\lambda|^{-1 / 2}(\theta, \lambda) \gamma_{\psi}(2 \lambda) \sum_{y \in R^{\times} /(1+\pi R)}(\lambda, y) \psi\left(-\left(y+y^{-1}\right) / \lambda\right) .
\end{aligned}
$$

Lemma 6.1. Let $k$ be a finite field of odd characteristic. Let $\tau$ be a nontrivial character of $k$. Put $\varepsilon(x)=1$ if $x$ is a square in $k^{\times}, \varepsilon(x)=-1$ if $x$ is not a square, $\varepsilon(0)=0$. Then

$$
\sum_{y \in k^{\times}} \varepsilon(y) \tau\left(y+y^{-1}\right)=(\tau(2)+\tau(-2)) \sum_{x \in k} \tau\left(x^{2}\right) .
$$

Apply this to $k=R / \pi R, \tau(x)=\psi\left(-\lambda^{-1} x\right)$. Then $\sum_{y \in R^{\times} /(1+\pi R)}(\lambda, y) \psi\left(-\left(y+y^{-1}\right) / \lambda\right)$ is equal to

$$
(\psi(2 / \lambda)+\psi(-2 / \lambda)) \sum_{x \in R / \pi R} \psi\left(-\lambda^{-1} x^{2}\right)=(\psi(2 / \lambda)+\psi(-2 / \lambda)) \gamma_{\psi}(-2 \lambda)|\lambda|^{-1 / 2} .
$$

Hence

$$
\Psi\left(\lambda, f^{\theta}\right)=|\lambda|^{-1}(\theta, \lambda)(\psi(2 / \lambda)+\psi(-2 / \lambda)) .
$$

Proof of Lemma. For $z \in k$, the quadratic equation $y+y^{-1}=z$ has (1) two solutions, both squares in $k^{\times}$, if both $z+2$ and $z-2$ are nonzero squares; (2) two solutions, none of which is a square, if neither $z+2$ nor $z-2$ is a square; (3) the solution $y=1$ if $z=2$; (4) the solution $y=-1$ if $z=-2$; and (5) no solutions if precisely one of $z+2, z-2$, is a square. Then

$$
\begin{aligned}
& \sum_{y \in k^{\times}} \varepsilon(y) \tau\left(y+y^{-1}\right)=\sum_{z \in k}(\varepsilon(z-2)+\varepsilon(z+2)) \tau(z) \\
= & \sum_{z \in k}(\varepsilon(z-2)+1) \tau(z)+\sum_{z \in k}(\varepsilon(z+2)+1) \tau(z) \\
= & \sum_{z \in k} \tau\left(x^{2}+2\right)+\sum_{z \in k} \tau\left(x^{2}-2\right)=(\tau(2)+\tau(-2)) \sum_{x \in k} \tau\left(x^{2}\right) .
\end{aligned}
$$

The lemma follows.

If $f^{\prime}$ is $f^{\prime 0}$ on $H$, and $\chi$ is unramified, we have $\Psi_{\chi}^{ \pm}\left(f^{\prime 0}\right)=1$, and $\Psi_{\chi}\left(f^{\prime 0}\right)$ is 0 if $\lambda \notin R$, 1 if $\lambda \in R^{\times}, 1+\psi\left(\lambda^{-1}\right)$ if $0<|\lambda|<1$. The proposition follows. 
6. Corresponding. The Fourier summation formula for $\mathbf{H}(\mathbb{A})=\mathrm{GL}(2, \mathbb{A})$ is an identity of sums of distributions. The geometric side is described by Proposition 4. The spectral side will be recorded next, following [F1], Section D. The notations are those of [F1], to be recalled below.

Proposition 7. For any $f^{\prime}=\otimes f_{v}^{\prime}$ on $\mathbf{H}(\mathbb{A})$ and a character $\chi$ of $\mathbb{A}^{\times} / F^{\times}$with $\chi^{2}=1$, the integral of Proposition 4 is equal to the sum of

$$
\sum_{\rho} \sum_{\Phi^{\prime} \in \rho} W_{\psi}\left(\rho\left(f^{\prime}\right) \Phi^{\prime}\right) \bar{L}_{\Phi^{\prime}}\left(\frac{1}{2}, \rho \otimes \chi\right)
$$

and

$$
4 \pi \sum_{\Phi^{\prime}} E_{\psi}\left(I\left(f^{\prime}, \chi, \frac{1}{2}\right) \Phi^{\prime}, \chi, \frac{1}{2}\right) \bar{\Phi}^{\prime}(1)
$$

and

$$
\frac{1}{2} \sum_{\omega} \sum_{\Phi^{\prime}} \int_{i \mathbb{R}} E_{\psi}\left(I\left(f^{\prime}, \omega, \zeta\right) \Phi^{\prime}, \omega, \zeta\right) \cdot \mathcal{L}_{\bar{\Phi}^{\prime}, \chi}\left(\omega^{-1}, \frac{1}{2}-\zeta\right) d \zeta
$$

where

$$
\mathcal{L}_{\Phi, \chi}(\omega, \zeta)=L_{\Phi}(\zeta, \omega \chi)^{2} L_{\Phi}\left(2 \zeta, \omega^{2}\right)^{-1} .
$$

Here $\rho$ ranges over all cuspidal irreducible representations of $\mathrm{PGL}(2, \mathbb{A}) ; \Phi^{\prime}$ ranges over an orthonormal basis of smooth vectors for the space of $\rho$ in $L_{0}^{2}(\mathbf{Z}(\mathbb{A}) H \backslash \mathbf{H}(\mathbb{A}))$. The Whittaker functional is defined by $W_{\psi}\left(\Phi^{\prime}\right)=\int_{N \backslash \mathbf{N}(\mathbb{A})} \Phi^{\prime}(u) \psi(u) d u$, and

$$
\begin{aligned}
L_{\Phi^{\prime}}(\zeta, \rho \otimes \omega) & =\int_{F^{\times} \backslash \mathbb{A}^{\times}} \Phi^{\prime}\left(\left(\begin{array}{cc}
a & 0 \\
0 & 1
\end{array}\right)\right)|a|^{\zeta-1 / 2} \omega(a) d^{\times} a \\
& =\int_{\mathbb{A}^{\times}} W_{\psi}\left(\rho\left(\left(\begin{array}{ll}
a & 0 \\
0 & 1
\end{array}\right)\right) \Phi^{\prime}\right)|a|^{\zeta-\frac{1}{2}} \omega(a) d^{\times} a
\end{aligned}
$$

is the $L$-function of $\rho \otimes \omega$ at $\zeta$ associated with $\Phi$. The $\omega$ range over a set of representatives for the set of connected components of the complex manifold of unitary characters $x \mapsto \omega(x)$ of $\mathbb{A}^{\times} / F^{\times}$, a connected component consisting of $\omega \nu^{i \zeta}, \nu(x)=|x|, \zeta \in \mathbb{R}(=$ field of real numbers). The normalizedly induced $\mathbf{H}(\mathbb{A})$-module $I(\omega, \zeta)$ consists of smooth $\Phi^{\prime}$ with

$$
\Phi^{\prime}\left(\left(\begin{array}{ll}
a & * \\
0 & b
\end{array}\right) g, \zeta\right)=\omega(a / b)|a / b|^{\zeta+1 / 2} \Phi^{\prime}(g, \zeta)
$$

and

$$
E_{\psi}\left(\Phi^{\prime}, \omega, \zeta\right)=\int_{N \backslash \mathbf{N}(\mathbb{A})} E\left(u, \Phi^{\prime}, \omega, \zeta\right) \psi(u) d u, \quad E\left(h, \Phi^{\prime}, \omega, \zeta\right)=\sum_{\delta \in B \backslash G} \Phi^{\prime}(\delta h, \zeta) .
$$


The sums in (7.2), (7.3) range over an orthonormal basis for $I(\omega, \zeta)$, independently of $\zeta$. All sums and integrals are absolutely convergent.

Our next aim is then to develop an analogous Fourier summation formula for the group $\mathbf{G}=\mathrm{GSp}(4)$. We shall do that on multiplying the spectral expression $K_{f}(u, h)$ for the kernel by $\bar{\psi}_{\theta}(u)$ and integrating over $u$ in $N \backslash \mathbf{N}(\mathbb{A})$ and $h$ in $C_{\theta} \mathbf{Z}(\mathbb{A}) \backslash \mathbf{C}_{\theta}(\mathbb{A})$.

It will be useful to fix conventions for induction. These should be the same as those used in the theory of Eisenstein series, which are used to describe the contribution to the kernel from its spectral decomposition, as recalled in the next few sections (in particular, in section 9). Thus let $F$ be a local field, $\rho$ an admissible representation of $\operatorname{GL}(2, F)$ with a trivial central character, $\omega$ a character of $F^{\times}$, and $\zeta_{2}$ a complex number. Then the $G=\mathbf{G}(F)$-module normalizedly induced from the data $\rho, \zeta_{2}, \omega$ on $P=\mathbf{P}(F)$ will be denoted by $I_{P}\left(\rho, \zeta_{2}, \omega\right)$. It consists of all smooth functions $\phi: G \rightarrow \rho$ which satisfy

$$
\phi\left(\left(\begin{array}{cc}
A & * \\
0 & \lambda w^{t} A^{-1} w
\end{array}\right) g\right)=|| A|/ \lambda|^{\zeta_{2}+\frac{3}{2}} \omega(|A| / \lambda) \rho(A)(\phi(g))
$$

$\left(A \in \mathrm{GL}(2, F),|A|=\operatorname{det} A, g \in G, \lambda \in F^{\times}\right)$.

If $\rho=I\left(\zeta_{1},-\zeta_{1}\right) \otimes \chi, \chi$ a character of $F^{\times}$with $\chi^{2}=1, \zeta_{1}$ a complex number, is the $\operatorname{GL}(2, F)$-module consisting of the smooth $\phi: \operatorname{PGL}(2, F) \rightarrow \mathbb{C}$ with $\phi\left(\left(\begin{array}{ll}a & * \\ 0 & b\end{array}\right) g\right)=$ $\chi(a b)|a / b|^{\zeta_{1}+1 / 2} \phi(g)$, then $I_{P}\left(\rho, \zeta_{2}, \omega\right)$ is $I_{B}\left(\zeta_{1}, \zeta_{2}-\zeta_{1}, \chi, \omega / \chi\right)$. Here $I_{B}\left(\zeta_{1}, \zeta_{2}, \chi, \omega\right)$ is the $G$-module induced from the Borel subgroup $B$, consisting of the smooth functions $\phi: G \rightarrow \mathbb{C}$ which satisfy

$$
\phi\left(\left(\begin{array}{ccc}
a & & * \\
& { }^{*} / b & \\
0 & & \lambda / a
\end{array}\right) g\right)=\left|\frac{a^{4} b^{2}}{\lambda^{3}}\right|^{1 / 2}\left|\frac{a^{2}}{\lambda}\right|^{\zeta_{1}}\left|\frac{a b}{\lambda}\right|^{\zeta_{2}} \chi\left(\frac{a^{2}}{\lambda}\right) \omega\left(\frac{a b}{\lambda}\right) \phi(g) \quad\left(a, b, \lambda \in F^{\times}\right) .
$$

If $\rho$ is a $\mathrm{GL}(2, F)$-module with central character $\omega_{\rho}$, and $\zeta_{3}$ a complex number, the induced $I_{Q}\left(\rho, \zeta_{3}\right)$ consists of $\phi: G \rightarrow \rho$,

$$
\phi\left(\left(\begin{array}{ccc}
a & * & * \\
0 & A & * \\
0 & 0 & \lambda / a
\end{array}\right) g\right)=\left|a^{2} / \lambda\right|^{1+\zeta_{3}} \omega_{\rho}^{-1}(a)(\rho(A) \phi)(g) \quad\left(A \in \mathrm{GL}(2, F), \lambda=|A|, a \in F^{\times}\right) .
$$

If $\rho=I\left(\zeta_{4},-\zeta_{4}\right) \otimes \chi$, then $\omega_{\rho}=\chi^{2}$, and $I_{Q}\left(\rho, \zeta_{3}\right)=I_{B}\left(\zeta_{3}-\zeta_{4}, 2 \zeta_{4}, \chi^{-1}, 1\right)$. Analogous notations will be used also in the global case .

It will be useful to compute the trace $\operatorname{tr} \pi(f)$ of $\pi=I\left(\zeta_{1}, \zeta_{2}\right)$. For this purpose take $\phi \in I\left(\zeta_{1}, \zeta_{2}\right)$, and $f \in C_{c}^{\infty}(Z \backslash G)$, and note that

$$
\begin{aligned}
(\pi(f) \phi)(h) & =\int_{Z \backslash G} f(g) \phi(h g) d g=\int_{N} \int_{Z \backslash A} \int_{K} f\left(h^{-1} n \tilde{a} k\right) \delta_{B}(\tilde{a})^{-1 / 2}\left|\frac{a^{2}}{\lambda}\right|^{\zeta_{1}}\left|\frac{a b}{\lambda}\right|^{\zeta_{2}} \phi(k) d n d \tilde{a} d k \\
& =\iiint \Delta(\tilde{a}) f\left(h^{-1} n^{-1} \tilde{a} n k\right)\left|\frac{a^{2}}{\lambda}\right|^{\zeta_{1}}\left|\frac{a b}{\lambda}\right|^{\zeta_{2}} \phi(k) d n d \tilde{a} d k,
\end{aligned}
$$


where

$$
\tilde{a}=\operatorname{diag}(a, b, \lambda / b, \lambda / a), \quad \delta_{B}(\tilde{a})=\left|a^{4} b^{2} / \lambda^{3}\right|,
$$

and

$$
\Delta(\tilde{a})=\delta_{B}(\tilde{a})^{-1 / 2}\left|\operatorname{det}(\operatorname{ad}(\tilde{a})-1)_{\operatorname{Lie} N}\right|=\frac{\left|(a-b)(a b-\lambda)\left(a^{2}-\lambda\right)\left(b^{2}-\lambda\right)\right|}{\left|a^{4} b^{4} \lambda^{3}\right|^{1 / 2}} .
$$

Put

$$
F_{f}(\tilde{a})=\Delta(\tilde{a}) \Phi_{f}(\tilde{a}), \quad \Phi_{f}(\tilde{a})=\int_{N} \int_{K} f\left(k^{-1} n^{-1} \tilde{a} n k\right) d k d n .
$$

Then

$$
\operatorname{tr} I\left(\zeta_{1}, \zeta_{2} ; f\right)=\int_{Z \backslash A} F_{f}(\tilde{a})\left|\frac{a^{2}}{\lambda}\right|^{\zeta_{1}}\left|\frac{a b}{\lambda}\right|^{\zeta_{2}} d \tilde{a} .
$$

When $f$ is $K$-biinvariant, $F_{f}(\tilde{a})$ depends only on the valuations of $a, b, \lambda$ in the nonarchimedean case. As usual $\pi$ denotes a generator of the maximal ideal in the ring of integers $R$ of $F, q$ the cardinality of the residue field $R /(\boldsymbol{\pi})$, and the absolute value is normalized by $|\boldsymbol{\pi}|=q^{-1}$. Put

$$
F_{f}(n, m)=F_{f}\left(\operatorname{diag}\left(1, \boldsymbol{\pi}^{n}, \boldsymbol{\pi}^{m}, \boldsymbol{\pi}^{m+n}\right)\right) .
$$

Then

$$
\operatorname{tr} I\left(\zeta_{1}, \zeta_{2} ; f\right)=\sum_{n} \sum_{m} q^{(m+n) \zeta_{1}} q^{m \zeta_{2}} F_{f}(n, m) .
$$

If $f^{\prime} \in C_{c}^{\infty}(Z \backslash H), H=\mathrm{GL}(2, F)$, and $F_{f^{\prime}}(\tilde{a})=\Delta_{H}(\tilde{a}) \Phi_{f^{\prime}}(\tilde{a}), \tilde{a}=\left(\begin{array}{cc}a & 0 \\ 0 & b\end{array}\right)$,

$$
\Delta_{H}(\tilde{a})=\left|(a-b)^{2} / a b\right|^{1 / 2} \text { and } \Phi_{f^{\prime}}(\tilde{a})=\int_{K^{\prime}} \int_{N^{\prime}} f^{\prime}\left(k^{-1} n^{-1} \tilde{a} n k\right) d n d k,
$$

we put $F_{f^{\prime}}(n)=F_{f^{\prime}}\left(\operatorname{diag}\left(\boldsymbol{\pi}^{-n}, 1\right)\right)$ if $f^{\prime}$ is $K^{\prime}$-biinvariant. A similar computation shows that

$$
\operatorname{tr} I_{H}\left(\zeta_{1},-\zeta_{1} ; f^{\prime}\right)=\int_{Z^{\prime} \backslash A^{\prime}} F_{f^{\prime}}(\tilde{a})\left|\frac{a}{b}\right|^{\zeta_{1}} d \tilde{a}=\sum_{n} F_{f^{\prime}}(n) q^{n \zeta_{1}},
$$

the last equality holds for a $K^{\prime}$-biinvariant function $f^{\prime}$.

Definition 7.1. The $K$-biinvariant function $f$ on $Z \backslash G$, and the $K^{\prime}$-biinvariant function $f^{\prime}$ on $Z \backslash H$, are corresponding, if $\operatorname{tr} I_{H}\left(\zeta_{1},-\zeta_{1} ; f^{\prime}\right)=\operatorname{tr} I\left(\zeta_{1}, \frac{1}{2}-\zeta_{1} ; f\right)$ for every complex number $\zeta_{1}$. In particular, the unit elements $f^{0}$ and $f^{\prime 0}$ are corresponding.

If $f^{\prime}$ and $f$ are corresponding, then

$$
\sum_{n} q^{n \zeta_{1}} F_{f^{\prime}}(n)=\sum_{n} \sum_{m} q^{(m+n) \zeta_{1}} q^{m\left(1 / 2-\zeta_{1}\right)} F_{f}(n, m)
$$

for all $\zeta_{1}$, hence

$$
F_{f^{\prime}}(n)=\sum_{m} q^{m / 2} F_{f}(n, m)
$$


Proposition 8. Corresponding $f^{\prime}$ and $f$ are matching.

This important statement was presented as a conjecture in an early draft of our paper. It was then proved by Zinoviev [Z], using Proposition 6, which establishes this statement in the case of $f^{\prime 0}$ and $f^{0}$. It will be interesting to find a simple and conceptual proof of our assertion, as one has in the case of base-change and ordinary orbital integrals. A suitable "symmetric space" analogue of the regular functions technique introduced in [F6] in the group case may yield a reduction of the spherical case (Proposition 8) to the germ expansion for a general test function, which is analyzed in Propositions 3 and 5, or to the case of the unit element (Proposition 6).

Remark. Since $I_{P}\left(\rho, \zeta_{2}\right)=I_{B}\left(\zeta_{1}, \zeta_{2}-\zeta_{1}\right)$ for $\rho=I_{H}\left(\zeta_{1},-\zeta_{1}\right)$, and $\operatorname{tr} \rho$ is invariant under the replacement $\zeta_{1} \mapsto-\zeta_{1}$, and since $I_{Q}\left(\rho, \zeta_{3}\right)=I_{B}\left(\zeta_{3}-\zeta_{4}, 2 \zeta_{4}\right)$ for $\rho=I\left(\zeta_{4},-\zeta_{4}\right)$, and $\operatorname{tr} \rho$ is invariant under $\zeta_{4} \mapsto-\zeta_{4}$, we conclude that $\operatorname{tr} I_{B}\left(\zeta_{1}, \zeta_{2}\right)$ is invariant under $\left(\zeta_{1}, \zeta_{2}\right) \mapsto\left(-\zeta_{1}, \zeta_{2}+2 \zeta_{1}\right)$, as well as under $\left(\zeta_{1}, \zeta_{2}\right) \mapsto\left(\zeta_{1}+\zeta_{2},-\zeta_{2}\right)$. Of course these relations are induced by the action of the two reflections in the Weyl group, $\alpha_{1}$ which is associated with the parabolic $P$, and $\alpha_{2}$ which is associated with $Q$.

7. Spectral analysis. The spectral expansion of the kernel $K_{f}(g, h)(g, h \in \mathbf{Z}(\mathbb{A}) \backslash \mathbf{G}(\mathbb{A}))$ of the convolution operator $r(f)$ on $L^{2}(\mathbf{Z}(\mathbb{A}) G \backslash \mathbf{G}(\mathbb{A}))$ is a sum of contributions associated to $G, P, Q$, and $B$. The contribution associated to $G$ is analogous to that described in the case of $H=\mathrm{GL}(2)$ above. Its cuspidal part is the bounded function

$$
K_{G}(g, h)=\sum_{\pi} m(\pi) \sum_{\Phi}(\pi(f) \Phi)(g) \bar{\Phi}(h) .
$$

The first sum ranges over a set of representatives for the set of equivalence classes of the cuspidal representations in $L^{2}(\mathbf{Z}(\mathbb{A}) G \backslash \mathbf{G}(\mathbb{A}))$. The multiplicity of $\pi$ in the cuspidal spectrum of $L^{2}(\mathbf{Z}(\mathbb{A}) G \backslash \mathbf{G}(\mathbb{A}))$ is a finite integer, denoted by $m(\pi)$. The inner sum ranges over an orthonormal basis for the space of $\pi$, consisting of smooth bounded functions $\Phi$. In the case of $H$, the multiplicity one theorem asserts that $m(\rho)$ are all equal to 1 . There we let $\rho$ range over the discrete spectrum representations, and $W_{\psi}\left(\Phi^{\prime}\right) \neq 0$ implied that $\rho$ is generic, hence cuspidal (not one dimensional). In the case of $G$, it has been shown in [F8] (note that $\mathrm{GSp}(4)$ of this paper is denoted by $\mathrm{GSp}(2)$ in $[\mathrm{F} 8]$ ) that the $m(\pi)$ are 1 for $G=\operatorname{PGSp}(4)$. Since $\psi_{\theta}$ is not a generic character of $N_{B}, W_{\psi_{\theta}}$ does not kill the non cuspidal discrete spectrum representations. Multiplying by $\bar{\psi}_{\theta}(g)$ and integrating over $g$ in $N \backslash \mathbf{N}(\mathbb{A})$ and $h$ in $\mathbf{Z}(\mathbb{A}) C_{\theta} \backslash \mathbf{C}_{\theta}(\mathbb{A}), \theta \in F$, we obtain

$$
\sum_{\pi} m(\pi) \sum_{\Phi} W_{\psi_{\theta}}(\pi(f) \Phi) P(\bar{\Phi})
$$

where

$$
W_{\psi_{\theta}}(\Phi)=\int_{N \backslash \mathbf{N}(\mathbb{A})} \Phi(n) \bar{\psi}_{\theta}(n) d n, \quad P(\Phi)=P_{\theta}(\Phi)=\int_{\mathbf{Z}(\mathbb{A}) C_{\theta} \backslash \mathbf{C}_{\theta}(\mathbb{A})} \Phi(h) d h .
$$


Note that the integral expression for $P(\Phi)$ converges also when $\Phi \in \pi, \pi$ a non cuspidal discrete spectrum (square integrable) representation. Indeed, by Schwartz inequality, for a closed subset $C$ of $G$ with finite volume and with characteristic function $1_{C}$ in $G$, we have that $\left(\int_{C}|f|\right)^{2}=\left(\int_{G}|f| \cdot 1_{C}\right)^{2} \leq \int_{G}|f|^{2} \cdot \int_{G} 1_{C}^{2} \leq \operatorname{vol}(C) \cdot \int_{G}|f|^{2}$ is finite. However, for such $\pi, P(\Phi)$ is redefined after the proof of Proposition 9 below (as the $\operatorname{limit} \lim P\left(\Lambda^{T} \Phi\right)$ ) to fit our proofs.

But we need to consider the non cuspidal spectrum as well. To deal with convergence questions, we briefly recall some consequences of Arthur's work [A1/2], mostly in his (standard) notations. This is best done in the context of a general reductive group $\mathbf{G}$ over $F$. Let $\mathbf{G}(\mathbb{A})^{1}$ denote the subgroup of the $g$ in $\mathbf{G}(\mathbb{A})$ with $|\chi(g)|=1$ for every rational character $\chi$ of $\mathbf{G}\left([\mathrm{A} 1]\right.$, p. 917). Put $\mathbb{K}=\prod_{v} K_{v}$, product over all places $v$ in $F$, of hyperspecial maximal compact subgroups $K_{v}$ of $\mathbf{G}\left(F_{v}\right)$. Let $f \in C_{c}^{\infty}(\mathbf{G}(\mathbb{A})$ ) be a $\mathbb{K}$-finite (the space spanned by its left and right $\mathbb{K}$-translates is finite dimensional) smooth compactly supported function on $\mathbf{G}(\mathbb{A})$. Denote by $\Lambda_{2}^{T}$ truncation ([A2], p. 89) with respect to the second variable, and by $\chi$ any class of cuspidal pairs ([A1], p. 924). Denote by $\mathbf{U}$ a closed $F$-subgroup of $\mathbf{G}$ such that $U \backslash \mathbf{U}(\mathbb{A})$ is compact, and by $\psi$ a character of $U \backslash \mathbf{U}(\mathbb{A})$ with $|\psi|=1$. Let $\mathbf{C}$ be a closed reductive $F$-subgroup of $\mathbf{G}$, such that $\mathbf{Z}(\mathbb{A}) C \backslash \mathbf{C}(\mathbb{A})$ has finite volume, where $\mathbf{Z}$ is the center of $\mathbf{G}$, and such that for any Siegel domain $S([\mathrm{HCM}],[\mathrm{PR}])$ in $\mathbf{G}(\mathbb{A})^{1}, S_{C}=S \cap \mathbf{C}(\mathbb{A})$ is a Siegel domain in $\mathbf{C}(\mathbb{A})$. We put $C=\mathbf{C}(F)$.

Proposition 9. Let $\omega$ be a compact set in $\mathbf{G}(\mathbb{A})^{1}$. Then for any sufficiently regular $([A 2]$, p. 89) $T$ in $\mathfrak{A}_{0}^{+}$we have $K_{f}(u, h)=\Lambda_{2}^{T} K_{f}(u, h)$ and $K_{f, \chi}(u, h)=\Lambda_{2}^{T} K_{f, \chi}(u, h)([A 1], p$. 935), for all $u \in \omega, h \in \mathbf{G}(\mathbb{A})$. For any Siegel domain $S$ in $\mathbf{G}(\mathbb{A})^{1}$ and $N>0$, there is $c>0$ such that $\sum_{\chi}\left|K_{f, \chi}(u, h)\right| \leq c\|h\|^{-N}$ for all $u \in \omega$ and $h \in S$. Consequently

$$
\int_{\mathbf{Z}(\mathbb{A}) C \backslash \mathbf{C}(\mathbb{A})} \int_{U \backslash \mathbf{U}(\mathbb{A})} K_{f}(u, h) \bar{\psi}_{\theta}(u) d u d h=\sum_{\chi} \iint K_{f, \chi}(u, h) \bar{\psi}_{\theta}(u) d u d h .
$$

Each side is finite even if $K \bar{\psi}$ is replaced by its absolute value. The Eisenstein series being defined in $[A 1]$, p. 926, put $E_{\psi}(\Phi, \pi)=\int_{U \backslash \mathbf{U}(\mathbb{A})} E(u, \Phi, \pi) \bar{\psi}_{\theta}(u) d u$. Then

$$
\sum_{P} n\left(A_{P}\right)^{-1} \int_{\Pi^{G}(M)}\left|\sum_{\Phi \in \mathfrak{B}_{P}(\pi)_{\chi}} E_{\psi}\left(I_{P}(\pi, f) \Phi, \pi\right) \int_{\mathbf{Z}(\mathbb{A}) C \backslash \mathbf{C}(\mathbb{A})} \Lambda^{T} \bar{E}(h, \Phi, \pi) d h\right| d \pi
$$

is finite. The expression obtained on erasing the absolute values is equal to

$$
\int_{\mathbf{Z}(\mathbb{A}) C \backslash \mathbf{C}(\mathbb{A})} \int_{U \backslash \mathbf{U}(\mathbb{A})} K_{f, \chi}(u, h) \bar{\psi}_{\theta}(u) d u d h .
$$

Proof. The truncation operator $\Lambda^{T}$ is defined in [A2], p. 89, to be (we put $|A / Z|$ for $\operatorname{dim}(A / Z))$

$$
\Lambda^{T} \phi(h)=\sum_{P}(-1)^{|A / Z|} \sum_{\delta \in P \backslash G} \hat{\tau}_{P}(H(\delta h)-T) \int_{N \backslash \mathbf{N}(\mathbb{A})} \phi(n \delta h) d n .
$$


Then

$$
\Lambda_{2}^{T} K_{f}(u, h)=\sum_{P}(-1)^{|A / Z|} \sum_{\delta \in P \backslash G} \hat{\tau}_{P}(H(\delta h)-T) \int_{N \backslash \mathbf{N}(\mathbb{A})} K_{f}(u, n \delta h) d n .
$$

Put $K_{P, f}(u, h)=\sum_{\mu \in M} \int_{\mathbf{N}(\mathbb{A})} f\left(u^{-1} \mu n h\right) d n$, as in [A1], p. 923. Then

$$
\int_{N \backslash \mathbf{N}(\mathbb{A})} K_{f}(u, n h) d n=\sum_{\gamma \in P \backslash G} K_{P, f}(\gamma u, h) .
$$

By [A2], p. 101, sentence including (2.4), if $K_{P, f}(\gamma u, \delta h) \neq 0$, then there exists $T_{0} \in \mathfrak{A}_{0}$, depending only on the compact support $\operatorname{supp}(f)$ of $f$, such that $\hat{\tau}_{P}\left(H(\gamma u)-H(\delta h)-T_{0}\right)=$ 1. By [A1], (5.2), p. 936, there is $c>0$ such that $\varpi(H(\gamma u)) \leq c(1+\ell n\|u\|)$ for all $u \in \mathbf{G}(\mathbb{A})^{1}, \gamma \in G, \varpi \in \hat{\Delta}_{0}$. Our $u$ lies in the compact $\omega$, hence there is some $c>0$ with $\varpi(H(\gamma u)) \leq c(u \in \omega, \gamma \in G)$, for all $\varpi \in \hat{\Delta}_{P}$. Hence $\varpi(H(\delta h))<c-\varpi\left(T_{0}\right)$, and $\hat{\tau}_{P}(H(\delta h)-T)$ is zero for a sufficiently regular $T$. Then the term indexed by $P \neq G$ vanishes, and $\Lambda^{T} K_{f}=K_{f}$. But the sentence including (2.4) on p. 101 of [A2] is valid also for $K_{P, f, \chi}$, for all $\chi$. Hence $\Lambda^{T} K_{f, \chi}=K_{f, \chi}$.

The kernel $K_{f, \chi}$ is defined in [A1], p. 935, to be

$$
K_{f, \chi}(u, h)=\sum_{P} n\left(A_{P}\right)^{-1} \int_{\Pi^{G}(M)} \sum_{\Phi \in \mathfrak{B}_{P}(\pi)_{\chi}} E\left(u, I_{P}(\pi, f) \Phi, \pi\right) \bar{E}(h, \Phi, \pi) d \pi .
$$

By [A1], Lemma 4.4, there is $N>0$ and a seminorm $\|\cdot\|_{0}$ on $C_{c}^{\infty}(\mathbf{G}(\mathbb{A}))$ such that

$$
\sum_{\chi} \sum_{P} n\left(A_{P}\right)^{-1} \int_{\Pi^{G}(M)}\left|\sum_{\Phi \in \mathfrak{B}_{P}(\pi)_{\chi}} E\left(u, I_{P}(\pi, f) \Phi, \pi\right) \bar{E}(h, \Phi, \pi)\right| d \pi \leq\|f\|_{0} \cdot\|u\|^{N} \cdot\|h\|^{N} .
$$

In particular $\sum_{\chi}\left|K_{f, \chi}(u, h)\right| \leq\|f\|_{0} \cdot\|u\|^{N} \cdot\|h\|^{N}$. By [A1], Corollary 5.2 (see also [A2], mid page 89), we can truncate $K_{f}(u, h)=\sum_{\chi} K_{f, \chi}(u, h)$ term by term: $\quad \Lambda_{2}^{T} K_{f}(u, h)$ $=\sum_{\chi} \Lambda_{2}^{T} K_{f, \chi}(u, h)$. Moreover, by [A1], Lemma 4.4, and [A2], Lemma 1.4, there exists some $N^{\prime}>0$ such that for any $N>0$ there is $c>0$ such that for all $u \in \mathbf{G}(\mathbb{A})^{1}$ and $h$ in a Siegel domain $S$, we have

$$
\sum_{\chi} \sum_{P} n\left(A_{P}\right)^{-1} \int_{\Pi^{G}(M)}\left|\sum_{\Phi \in \mathfrak{B}_{P}(\pi)_{\chi}} E\left(u, I_{P}(\pi, f) \Phi, \pi\right) \Lambda^{T} \bar{E}(h, \Phi, \pi)\right| d \pi \leq c\|u\|^{N^{\prime}}\|h\|^{-N} .
$$

Hence

$$
\sum_{\chi}\left|K_{f, \chi}(u, h)\right|=\sum_{\chi}\left|\Lambda_{2}^{T} K_{f, \chi}(u, h)\right| \leq c|| h \|^{-N}
$$


for $u \in \omega$ and $h \in S$, and the proposition follows.

In particular, the contribution associated to $P=G$ is (8.1), but the first sum ranges over all discrete spectrum representations of $\mathbf{G}(\mathbb{A})$, not only the cuspidal ones. For a $\mathbb{K}$ finite function $f$ and a fixed $\pi$, the sum over $\Phi$ is finite (depending on $\mathbb{K}$, but not $f$ ). Since the Hecke algebra of $\mathbb{K}$-biinvariant functions generate the algebra of endomorphisms of $\pi^{\mathbb{K}}$, the absolute convergence of the sentence before last in Proposition 9, and the explicit computations below of all terms in that expression for $P \neq G$, imply the convergence of $\lim P\left(\Lambda^{T} \Phi\right)$, where $\Phi \in \pi, \pi$ being a non cuspidal discrete spectrum representation, as $T \rightarrow \infty$. We define $P(\Phi)$ to be this limit. We say that such $\pi$ is cyclic if $P(\Phi) \neq 0$ for some $\Phi \in \pi$.

By [A1], (3.1), p. 928, the Eisenstein series $E(x, \Phi, \zeta)$, and each of its derivatives in $x$, is bounded by $c(\zeta)\|x\|^{N}(x \in \mathbf{G}(\mathbb{A}))$, where $c(\zeta)$ is a locally bounded function on the set of $\zeta \in \mathfrak{A}_{\mathbf{C}(\mathbb{A})}^{*}$ where $E(x, \Phi, \zeta)$ is regular. Let us review the well known fact that on $i \mathfrak{A}^{*}$, where $E(x, \Phi, \zeta)$ is regular, it has polynomial growth in $\zeta$. For this purpose, embed $\mathbb{R}_{>0}^{\times}$in $\mathbb{A}^{\times}$via $x \mapsto(x, \ldots, x, 1, \ldots)(x$ in the archimedean components, 1 in the finite components). Put (as in [A1], p. 925) $\Pi=\operatorname{Hom}_{\text {cts }}\left(\mathbb{A}^{\times} / F^{\times} \mathbb{R}_{>0}^{\times}, S^{1}\right)$, where $S^{1}$ is the unit circle in the complex plane, and $\Pi_{0}=\operatorname{Hom}_{\mathrm{cts}}\left(\mathbb{A}^{\times} / F^{\times} \mathbb{R}_{>0}^{\times} U, S^{1}\right)$, where $U=\prod_{v} U_{v}$, and $U_{v}$ is the maximal compact subgroup of $F_{v}^{\times}$. If $v_{j}(1 \leq j \leq r)$ are the archimedean places of $F$, for $\mu \in \Pi_{0}$ we have $\mu\left(z_{v_{j}}\right)=\left|z_{v_{j}}\right| \mu_{v_{j}}^{\mu_{j}}, \mu_{j} \in i \mathbb{R}$, with $\sum_{j} \mu_{j}\left[F_{v_{j}}: \mathbb{R}\right]=0$. These $\mu_{j}\left(\mu \in \Pi_{0}\right)$ form a discrete subgroup of rank $r-1$ in this hyperplane. Denote by $C_{0}(\mu)$ a function on $\Pi_{0}$ of the form $C_{0}(\mu)=c \prod_{j}\left(1-\mu_{j}^{2}\right)^{c_{j}}$ with $c>0, c_{j}>0$. In fact it depends only on the restriction of $\mu$ to $F^{\times} U F_{\infty}^{\times}$, where $F_{\infty}^{\times}=\prod_{j} F_{j}^{\times}$.

Choose a set of representatives $\tilde{\mu}$ for $\Pi / \Pi_{0}$, and a function $C_{\tilde{\mu}}$ on $\Pi_{0}$ of the above type for each $\tilde{\mu}$. Denote by $C(\mu)$ the function on $\Pi$ defined by $C(\mu)=C_{\tilde{\mu}}(\mu / \tilde{\mu})$ if $\mu=\tilde{\mu}$ on $U$; then $C(\mu)$ depends only on the restriction of $\mu$ to $F^{\times} U F_{\infty}^{\times}$. Denote by $c(\mu)$ a non negative valued function on $\Pi$ which depends only on the restriction of $\mu$ to $U$. Using the existence of zero free regions of $L$-functions about $\operatorname{Re}(\zeta)=1$, we have:

Lemma 9.1. There are functions $C_{1}(\mu), C_{2}(\mu), c_{1}(\mu), c_{2}(\mu)$ as above, such that for complex $\zeta$ with $|\operatorname{Re} \zeta| \leq C_{1}(\mu)^{-1}\left(1+(\operatorname{Im} \zeta)^{2}\right)^{-c_{1}(\mu)}$ we have that $|L(\zeta, \mu) / L(1+\zeta, \mu)|$ is bounded by $C_{2}(\mu)\left(1+(\operatorname{Im} \zeta)^{2}\right)^{c_{2}(\mu)}$ (a bound of the same type holds for any derivative of the quotient, by Cauchy's integral formula).

Proof. For a complex number $s=\sigma+i t$, put $L_{f}(s, \mu)=\prod_{v<\infty} L\left(s, \mu_{v}\right)$. This $L_{f}(s, \mu)$ converges absolutely on $\sigma \geq 1+\delta, \delta>0$, by [La], p. 158. It has analytic continuation to the entire complex plane, and it has no zeroes on $\sigma=1$. For any vertical strip of finite width there are $C(\mu)$ and $c(\mu)$ such that for all $\mu$, and $s$ with $\sigma$ in the strip, $\left|L_{f}(s, \mu)\right|$ is bounded by $C(\mu)(1+s \bar{s})^{c(\mu)}$. In fact, by [La], p. 334, for any $t_{0}>0$ there is $m>0$ such that $s(s-1) L_{f}(s, \mu)$ is $O\left(|t|^{m}\right)$ in the vertical strip $-1<\sigma<2,|t|>t_{0}$. Then $\left|L_{f}(s, \mu)\right|<$ $C_{1}^{-1}|t|^{m}$ in this strip, and by Cauchy's integral formula we also have $\left|L_{f}^{\prime}(s, \mu)\right|<C_{1}^{-1}|t|^{m}$ there. Take $\varepsilon_{0}>0$ such that $\left|L_{f}(s, \mu)\right|>C_{2}$ in $|t| \leq 1,|\sigma-1| \leq \varepsilon_{0}$. Here $C_{i}$ are positive constants. As in [La], p. 313, one has $\left|L_{f}(\sigma, 1)^{3} L_{f}(\sigma+i t, \mu)^{4} L_{f}\left(\sigma+2 i t, \mu^{2}\right)\right| \geq 1$ on $\sigma>1$. Hence $\left|L_{f}(s, \mu)\right| \geq\left|L_{f}(\sigma, 1)\right|^{-3 / 4}\left|L_{f}\left(\sigma+2 i t, \mu^{2}\right)\right|^{-1 / 4}>C_{3}|\sigma-1|^{3 / 4}|t|^{-m / 4}$ on $\sigma>1$, 
$|t| \geq 1$. Put $C_{4}=\left(C_{1} C_{3} / 3\right)^{4}$, and $m^{\prime}=6 m$. Given $\zeta$ with $1-C_{4}|t|^{-m^{\prime}}<\operatorname{Re} \zeta \leq 1$, put $s=1+C_{4}|t|^{-m^{\prime}}+i t$. Then $\left|L_{f}(s, \mu)-L_{f}(\sigma, \mu)\right|=\left|\int_{\operatorname{Re} \zeta}^{\operatorname{Re} s} L_{f}^{\prime}(u+i t, \mu) d u\right|$ is bounded by $C_{1}^{-1}|t|^{m}(\operatorname{Re} s-\operatorname{Re} \zeta) \leq 2\left(C_{4} / C_{1}\right)|t|^{m-m^{\prime}}$. By the triangle inequality,

$$
\left|L_{f}(\zeta, \mu)\right| \geq\left|L_{f}(s, \mu)\right|-\left|L_{f}(s, \mu)-L_{f}(\zeta, \mu)\right| \geq C_{3} C_{4}^{3 / 4}|t|^{-\left(3 m^{\prime}+m\right) / 4}-2\left(C_{4} / C_{1}\right)|t|^{m-m^{\prime}}
$$

on $|\operatorname{Im} \zeta| \geq 1$. Since $\left|L_{f}(\zeta, \mu)\right|>C_{2}$ in $|\operatorname{Im} \zeta| \leq 1,|\operatorname{Re} \zeta-1| \leq \varepsilon_{0}$, we are done (replacing $C_{i}$ by $C_{i}(\mu)$ and $m$ by $c(\mu)$, and using Stirling's formula to bound the ratio of the gamma factors at infinity).

Note that for characters $\mu$ of finite order, much better estimates are known: $\operatorname{Im} \zeta$ can be replaced by $\ell n \operatorname{Im} \zeta$ in our estimates. But we need here only our crude estimates.

Lemma 9.2. Let $\pi$ be a unitary $\mathbf{G}(\mathbb{A})$-module on a Hilbert space $H$. Let $H^{0}$ be the subspace of $\mathbb{K}$-finite vectors. Suppose that each $\mathbb{K}$-type has finite multiplicity. Let $L_{1}, L_{2}$ be linear forms on $H^{0}$. Let $f$ be $\mathbb{K}$-finite in $C_{c}^{\infty}(\mathbf{G}(\mathbb{A}))$, and $\{\phi\}$ an orthonormal basis of $H^{0}$. Then the sum $\sum_{\{\phi\}} L_{1}(\pi(f) \phi) \overline{L_{2}(\phi)}$ is independent of the choice of the orthonormal basis $\{\phi\}$. In particular, if $f=f_{1} * f_{2}^{*}, f_{2}^{*}(g)=\overline{f_{2}\left(g^{-1}\right)}, f_{1}$ and $f_{2}$ are $\mathbb{K}$-finite elements of $C_{c}^{\infty}(\mathbf{G}(\mathbb{A}))$, then $\sum_{\{\phi\}} L_{1}\left(\pi\left(f_{1}\right) \phi\right) \overline{L_{2}\left(\pi\left(f_{2}\right) \phi\right)}=\sum_{\{\phi\}} L_{1}(\pi(f) \phi) \overline{L_{2}(\phi)}$.

From now on $\mathbf{G}$ is our GSp(4). Suppose that $f=f_{1} * f_{2}^{*}$, where $f_{2}^{*}(g)=\overline{f_{2}\left(g^{-1}\right)}$, and $f_{1}, f_{2}$ are $\mathbb{K}$-finite elements of $C_{c}^{\infty}(\mathbf{G}(\mathbb{A}))$. We will consider - for a sufficiently regular $T$ - the integral $\iint \Lambda^{T} K_{c} \bar{\psi}$, which - by the elementary Lemma 9.2 - is equal to

$$
\begin{gathered}
\sum_{P_{1}} n\left(A_{P_{1}}\right)^{-1} \sum_{\rho} \int_{i \mathfrak{A}_{P_{1}}^{*}} \sum_{\Phi} E_{\theta}\left(I\left(f_{1}\right) \Phi, \rho, \zeta\right) \\
\cdot \int_{\mathbf{Z}(\mathbb{A}) C_{\theta} \backslash \mathbf{C}_{\theta}(\mathbb{A})} \Lambda^{T} \bar{E}\left(h, I\left(f_{2}\right) \Phi, \rho, \zeta\right) d h d \zeta,
\end{gathered}
$$

where $I(f)=I(f, \rho, \zeta)$ and $E_{\theta}(\Phi, \rho, \zeta)=\int_{N \backslash \mathbf{N}(\mathbb{A})} E(u, \Phi, \rho, \zeta) \bar{\psi}_{\theta}(u) d u$. The sum over the $P_{1}$ ranges over the standard parabolic subgroups: $\mathbf{G}$, the Siegel parabolic $\mathbf{P}$, the parabolic $\mathbf{Q}$ with the Heisenberg unipotent radical, and the minimal parabolic $\mathbf{B}$. The $\rho$ range over a set of representatives for the equivalence classes of discrete spectrum representations of $\mathbf{M}_{1}(\mathbb{A})^{1}$, where $\mathbf{M}_{1}$ is the standard Levi factor of $\mathbf{P}_{1}$. Put $\mathbb{K}_{\theta}=\prod_{v} K_{\theta, v}$, where $K_{\theta, v}$ is the standard maximal compact subgroup in $\mathbf{C}_{\theta}\left(F_{v}\right)$.

Lemma 9.3. There exists a hyperspecial maximal compact subgroup $\mathbb{K}=\prod_{v} K_{v}$ in $\mathbf{G}(\mathbb{A})$ which is adapted to $\mathbf{C}_{\theta}(\mathbb{A})$ and to $\mathbf{M}_{P}(\mathbb{A})$, in the sense that $K_{\theta, v}=K_{v} \cap C_{\theta, v}$ for all $v$, and $\operatorname{diag}(A, \lambda \varepsilon A \varepsilon)$ lies in $K_{v}$ precisely when $A$ lies in the standard maximal compact subgroup of $\mathrm{GL}\left(2, F_{v}\right)$, and $|\lambda|_{v}=1$, for all $v$.

Proof. Recall from section 2 that $\mathbf{C}_{\theta}$ is the centralizer in $\mathbf{G}$ of $\Theta_{\theta}=\gamma \sqrt{\theta} \Theta \gamma^{-1}$, where $\gamma=d\left(\left(\begin{array}{cc}1 & 1 \\ \sqrt{\theta} & -\sqrt{\theta}\end{array}\right)\right), d(A)=\operatorname{diag}(A, \varepsilon A \varepsilon)$. If $\theta$ is a square in $F_{v}$, then

$$
C_{\theta, v}=\gamma\left(\mathrm{GL}\left(2, F_{v}\right) \times \mathrm{GL}\left(2, F_{v}\right)\right) \gamma^{-1} .
$$


If $v$ is nonarchimedean, take $K_{v}$ to be the intersection of $\operatorname{GSp}\left(F_{v}\right)$ and the stabilizer of the lattice with basis $e_{1} \pm \sqrt{\theta} e_{2}, e_{3} \pm \sqrt{\theta} e_{4}$. When $F_{v}=\mathbb{R}$, take $K_{v}$ to be the intersection of $\operatorname{GSp}(\mathbb{R})$ and the orthogonal group of the quadratic form with matrix diag $(\theta, 1, \theta, 1)$. When $F_{v}=\mathbb{C}$, take $K_{v}$ to be the intersection of $\operatorname{GSp}(\mathbb{C})$ and the unitary group of the hermitian form with matrix $\operatorname{diag}(|\theta|, 1,|\theta|, 1)$. Then $K_{v} \cap C_{\theta, v}$ is $\gamma\left(\operatorname{GL}\left(2, R_{v}\right) \times \operatorname{GL}\left(2, R_{v}\right)\right) \gamma^{-1}$ $\left(R_{v}\right.$ is the ring of integers in $\left.F_{v}\right), \gamma(\mathrm{O}(2) \times \mathrm{O}(2)) \gamma^{-1}$, or $\gamma(\mathrm{U}(2) \times \mathrm{U}(2)) \gamma^{-1}$. Moreover, $\operatorname{diag}(A, \lambda \varepsilon A \varepsilon)$ lies in $K_{v}$ precisely when $|\lambda|_{v}=1$ and $A$ lies in the stabilizer of the lattice with basis $e_{1} \pm \sqrt{\theta} e_{2}$, the orthogonal group of $\operatorname{diag}(\theta, 1)$, or the unitary group of $\operatorname{diag}(|\theta|, 1)$, respectively.

If $\theta$ is not a square in $F_{v}$, then $C_{\theta, v}=\mathrm{GL}\left(2, E_{v}\right)^{\prime}$, where $E_{v}=F_{v}(\sqrt{\theta})$. When $v$ is nonarchimedean, choose $\alpha, \beta$ in $F_{v}$ such that 1 and $\alpha+\beta \sqrt{\theta}$ make a basis of the ring $R_{v}^{\prime}$ of integers in $E_{v}$, over $R_{v}$. For almost all $v$ we may and do choose $\alpha=0, \beta=1$. Take $K_{v}$ to be the intersection of $\operatorname{GSp}\left(F_{v}\right)$ and the stabilizer of the lattice $\Lambda$ with basis $e_{1}$, $\alpha e_{1}+\beta \theta e_{2}, e_{3}, \alpha e_{3}+\beta \theta e_{4}$. Then $K_{v} \cap C_{\theta, v}=\mathrm{GL}\left(2, R_{v}^{\prime}\right)^{\prime}$. Moreover, $\operatorname{diag}(A, \lambda \varepsilon A \varepsilon)$ lies in $K_{v}$ precisely when $|\lambda|_{v}=1$ and $A$ lies in the stabilizer of the lattice with basis $e_{1}, \alpha e_{1}+\beta \theta e_{2}$. Indeed, if $h=\left(\begin{array}{ll}a & b \\ c & d\end{array}\right), a=\left(\begin{array}{cc}a_{1} & a_{2} \\ \theta a_{2} & a_{1}\end{array}\right), \ldots, d=\left(\begin{array}{cc}d_{1} & d_{2} \\ \theta d_{2} & d_{1}\end{array}\right)$, and $m_{i}=\left(\begin{array}{cc}a_{i} & b_{i} \\ c_{i} & d_{i}\end{array}\right), i=1,2$, then $\operatorname{det} h=\left(\operatorname{det} m_{1}+\theta \operatorname{det} m_{2}\right)^{2}-u^{2} \theta$, where $u=a_{1} d_{2}+a_{2} d_{1}-b_{1} c_{2}-b_{2} c_{1}$ is 0 precisely when $h \in C_{\theta, v}$. Then each of the conditions $h \Lambda=\Lambda$ and $\left(\begin{array}{l}\mathbf{a} \mathbf{b} \\ \mathbf{c} \mathbf{d}\end{array}\right) \in \operatorname{GL}\left(2, R_{v}^{\prime}\right)$, where $\mathbf{a}=a_{1}+a_{2} \sqrt{\theta}, \cdots, \mathbf{d}=d_{1}+d_{2} \sqrt{\theta}$, is equivalent to $m_{1}+(\alpha / \beta) m_{2} \in M_{2}\left(R_{v}\right), \beta^{-1} m_{2} \in$ $M_{2}\left(R_{v}\right)$, $\operatorname{det}\left(m_{1}\right)+\theta \operatorname{det}\left(m_{2}\right) \in R_{v}^{\times}$. Finally, if $F_{v}=\mathbb{R}$, take $K_{v}$ to be the intersection of $\operatorname{GSp}(\mathbb{R})$ and the orthogonal group of the quadratic form with matrix $\operatorname{diag}(|\theta|, 1,|\theta|, 1)$. Then $K_{v} \cap C_{\theta, v}$ is $\mathrm{GL}(2, \mathbb{C}) \cap \mathrm{U}(2)$. Moreover, $\operatorname{diag}(A, \lambda \varepsilon A \varepsilon)$ lies in $K_{v}$ precisely when $|\lambda|_{v}=1$ and $A$ lies in the orthogonal group of $\operatorname{diag}(|\theta|, 1)$. Note that when $E_{v} / F_{v}$ is ramified, the image of $\mathrm{GL}\left(2, R_{v}^{\prime}\right)^{\prime}$ in $\mathrm{GL}\left(2, E_{v}\right)^{\prime} / F_{v}^{\times}$is not a maximal compact subgroup (it has index 2 in such a subgroup), but it can still be used.

As in [A1], p. 925, let $H^{0}(\rho)$ be the space of functions $\Phi$ on $\mathbf{Z}(\mathbb{A}) \mathbf{N}(\mathbb{A}) M \backslash \mathbf{G}(\mathbb{A})$ which are right $\mathbb{K}$-finite such that $m \mapsto \Phi(m g)$ is a matrix coefficient of $\rho$ for all $g$ in $\mathbf{G}(\mathbb{A})$, and $\Phi$ is square integrable on $\mathbb{K} \times M_{1} \backslash \mathbf{M}_{1}(\mathbb{A})^{1}$. Denote by $I(\rho, \zeta)$ the $\mathbf{G}(\mathbb{A})$-module normalizedly induced from the $\mathbf{P}_{1}(\mathbb{A})$-module $\rho_{\zeta}=\mu \otimes \delta_{P_{1}}^{\zeta}$. The Eisenstein series, for $\zeta \in \mathfrak{A}_{P_{1}, \mathbb{C}}^{*}$ with real part $\operatorname{Re} \zeta \in \rho_{P_{1}}+\left(\mathfrak{A}_{P_{1}}^{*}\right)^{+}$, is defined by the absolutely convergent series

$$
E(g, \Phi, \rho, \zeta)=\sum_{\gamma \in P_{1} \backslash G} \Phi(\gamma g, \zeta), \quad \Phi(g, \zeta)=\Phi(g) \delta_{P_{1}}(g)^{\zeta+\frac{1}{2}} .
$$

It has analytic continuation which is holomorphic on $i \mathfrak{A}_{P_{1}}^{*}$.

Let $M(w, \zeta) \Phi$ be the image of $\Phi$ under the action of the intertwining operator $M(w, \zeta)=$ $M(w, \rho, \zeta)$, associated with the Weyl group element $w$ ([A1], p. 926). As $\Phi(g, \rho, \zeta)$ lies in the induced $I(\rho, \zeta)$, the function $(M(w, \zeta) \Phi)\left(g,{ }^{w} \rho, w \zeta\right)$ lies in the induced $I\left({ }^{w} \rho, w \zeta\right)$. The operator $M(w, \zeta)$ has no singularity on the imaginary axis.

A $\mathbb{K}$-type $\kappa$ is a finite set of equivalence classes of irreducible $\mathbb{K}$-modules. The norm of the intertwining operator $M(\rho, \zeta)$ on the $\kappa$-component of $I(\rho, \zeta)$ is denoted by $\|M(\rho, \zeta)\|_{\kappa}$. In 
the next Proposition we take $\mathbf{P}_{1}$ to be $\mathbf{B}=\mathbf{A} \mathbf{U}$, the upper triangular subgroup, where $\mathbf{A}$ is the diagonal subgroup and $\mathbf{U}$ is the unipotent upper triangular subgroup. The $\kappa$-component of $I(\rho, \zeta)$ is zero unless the restriction of $\rho$ to $U$ lies in a finite set depending on $\kappa$.

Proposition 9.4. (1) Fix a $\mathbb{K}$-type $\kappa$. There are functions $C_{j}(\rho), c_{j}(\rho)$, such that for any complex $\zeta$ in the set $\Omega$ defined by $|\operatorname{Re} \zeta| \leq C_{1}(\rho)^{-1}\left(1+(\operatorname{Im} \zeta)^{2}\right)^{-c_{1}(\rho)}$, we have that $\|M(\rho, \zeta)\|_{\kappa}$ is bounded by $C_{2}(\rho)\left(1+(\operatorname{Im} \zeta)^{2}\right)^{c_{2}(\rho)}$. A bound of the same type holds for any derivative of the intertwining operator.

(2) Given $\kappa$, there are $C_{j}(\rho), c_{j}(\rho)$, such that for any $\zeta$ in $\Omega$, and for any $\Phi$ in the $\kappa$ component of $I(\rho, \zeta)$, the integral $\int_{G \backslash \mathbf{G}(\mathbb{A})}\left|\Lambda^{T} E(g, \Phi, \rho, \zeta)\right|^{2} d g$ is bounded by the product of $\|\Phi\|, C_{2}(\rho)\left(1+(\operatorname{Im} \zeta)^{2}\right)^{c_{2}(\rho)}$, and $\exp \left(c_{3}(\rho)\|T\|\right)$.

(3) For any $\mathbb{K}$-finite $f \in C_{c}^{\infty}(\mathbf{G}(\mathbb{A}))$ there are $C_{j}(\rho), c_{j}(\rho)$, such that for any $\zeta$ in $\Omega$, $x \in \mathbf{G}(\mathbb{A})^{1}$, we have that $|E(g, I(\rho, \zeta ; f) \Phi, \rho, \zeta)|$ is bounded by the product of $\|\Phi\|, C_{2}(\rho)(1+$ $\left.(\operatorname{Im} \zeta)^{2}\right)^{c_{2}(\rho)}$, and $\|g\|^{c_{3}(\rho)}$. The same holds for any derivative in $\zeta$ of this function.

Proof. The intertwining operator $M$ is the product of a normalized intertwining operator, which is easily majorized, a factor of absolute value one, and a product of quotients of $L$-functions of the type which appears in Lemma 9.1. (1) follows. (2) follows from this, via the scalar product formula of [A2], Lemma 4.2, p. 119 (see also [A5]).

For (3), note that in general, given a compact $\omega_{1}$ in $\mathbf{G}(\mathbb{A})^{1}$, we have $\Lambda^{T} \Phi(g)=\Phi(g)$ for any $g \in \omega_{1}$ and any function $\Phi$, provided that $T$ is sufficiently regular with respect to $\omega_{1}$. Indeed, [A1], (5.2), p. 936, asserts that there is a constant $c>0$ such that for any $\varpi \in \hat{\Delta}$, $\gamma \in G$, and $g \in \mathbf{G}(\mathbb{A})^{1}$, we have $\varpi(H(\gamma g)) \leq c(1+\ell n\|g\|)$. It suffices to take $T$ with $\varpi(T) \geq c(1+\ell n\|g\|)$ for all $\varpi \in \hat{\Delta}$ and $g \in \omega_{1}$. In fact we take $T_{1}$ with $\varpi\left(T_{1}\right) \geq c$ for all $\varpi \in \hat{\Delta}$, and $T=T_{1} \cdot \max \left\{1+\ell n\|g\| ; g \in \omega_{1} \cdot \operatorname{supp}(f)\right\}$. Then $\Lambda^{T} \Phi(g)=\Phi(g)$ for all $g$ in the compact $\omega_{1} \cdot \operatorname{supp}(f)$, and $\|T\| \leq c_{1} \max \left\{1+\ell n\|g\| ; g \in \omega_{1}\right\}$ for some $c_{1}=c_{1}(f)>0$. For these $f, \omega_{1}$, and $T$, we have for all $g \in \omega_{1}$,

$$
\begin{aligned}
& E(g, I(\rho, \zeta ; f) \Phi, \rho, \zeta)=\int_{\mathbf{G}(\mathbb{A})} E(g h, \Phi, \rho, \zeta) f(h) d h \\
& =\int_{\mathbf{G}(\mathbb{A})} \Lambda^{T} E(g h, \Phi, \rho, \zeta) f(h) d h=\int_{G \backslash \mathbf{G}(\mathbb{A})} \Lambda^{T} E(h, \Phi, \rho, \zeta) K_{f}(g, h) d h,
\end{aligned}
$$

where $K_{f}(g, h)=\sum_{\gamma \in G} f\left(g^{-1} \gamma h\right)$. But $\left|K_{f}(g, h)\right| \leq c_{2}\|g\|^{N}$, and (2) gives an $L^{2}$-bound for $\Lambda^{T} E$. Hence the expression to be estimated is bounded by the product of $\|\Phi\|, C(\rho)(1+$ $\left.(\operatorname{Im} \zeta)^{2}\right)^{c_{1}(\rho)}$, and $\max \left(\|g\|^{c_{3}(\rho)}\right)$. The maxima are taken over $x$ in the compact $\omega_{1}$. Finally, taking $\omega$ to be a compact neighborhood of the identity, we observe that for any $x \in \mathbf{G}(\mathbb{A})^{1}$, $\max (\|g\|)$ is bounded on $\omega_{1}=x \omega$ by a multiple of $\max (\|x\|)$, and (3) follows.

8. Summation formula. Let $V$ be a finite set of $F$-places, containing the archimedean places and those which ramify in $E$. A superscript $V$ will mean: "without $V$-component", e.g.: $\mathbb{A}^{\times, V}, \mathbb{A}_{E}^{\times, V}, \mathbb{A}^{V}, \chi^{V}$ (for a character $\chi$ on $\mathbb{A}^{\times}$), $U^{V}=\left\{t \in \mathbb{A}^{\times, V} ;\left|t_{v}\right|_{v}=1\right.$ for all $v \notin$ $V\}$. We write $E_{V}$ for the product of $E_{v}$ over $v \in V$, and $F_{V}=\prod_{v \in V} F_{v}$. Put $d(A)=$ 
$\operatorname{diag}(A, \varepsilon A \varepsilon)$, and

$$
\mathcal{L}_{V}(s, \rho, \zeta, \Phi)=\int_{E_{V}^{\times} / F_{V}^{\times}} d A \int_{\mathbb{K}_{\theta}} e^{\left\langle\rho_{0}+s \zeta, H_{0}(d(A) k)\right\rangle}(M(s, \rho, \zeta) \Phi)(d(A) k) d k .
$$

Here $M(s, \rho, \zeta)$ is the standard intertwining operator associated with $s \in W$.

The Fourier summation formula for $\mathbf{G}(\mathbb{A})=\operatorname{GSp}(4, \mathbb{A})$ is the following.

Proposition 10. Suppose that $f=f_{1} * f_{2}^{*}$, where $f_{2}^{*}(g)=\bar{f}_{2}\left(g^{-1}\right)$, and $f_{1}, f_{2}$ are $\mathbb{K}$-finite elements of $C_{c}^{\infty}(\mathbf{G}(\mathbb{A}))$ which are spherical ( $K_{v}$-biinvariant) outside $V$. Then the integral of (2.1) is equal to the sum (8.1) + (10.1), except that now the sum over $\pi$ in (8.1) ranges over the equivalence classes of discrete spectrum representations of $\mathbf{G}(\mathbb{A})$. Here

$$
\begin{aligned}
& \frac{1}{8} \sum_{\omega} \int_{i \mathbb{R}}\left[\sum_{\Phi} E_{\theta}\left(I\left(f,(1, \omega),\left(\frac{1}{2}, \zeta-\frac{1}{2}\right)\right) \Phi,(1, \omega),\left(\frac{1}{2}, \zeta-\frac{1}{2}\right)\right)\right. \\
& \left.\cdot \mathcal{L}^{V}\left(\omega^{-1}, \frac{1}{2}-\zeta\right) \cdot \overline{\mathcal{L}}_{V}\left(\gamma_{0},(1, \omega),\left(\frac{1}{2}, \zeta-\frac{1}{2}\right), \Phi\right)\right] d \zeta .
\end{aligned}
$$

The sum ranges over the unitary characters $\omega$ of $\mathbb{A}^{\times} / F^{\times} U \mathbb{R}_{+}^{\times}$. The Eisenstein series is associated with the character $h=(a, b, \lambda / b, \lambda / a) \mapsto\left|a^{2} / \lambda\right|^{1 / 2}|a b / \lambda|^{\zeta-\frac{1}{2}} \omega(a b / \lambda)$ of the diagonal subgroup. Here $\gamma_{0}$ represents the reflection (23). The sum (10.1), in which the brackets $[\cdot]$ are replaced by the absolute value $|\cdot|$, is convergent.

The proof of this Proposition occupies the next three sections.

The function $\mathcal{L}^{V}(\chi, \zeta)$ is defined and studied in the next Proposition. Its definition involves the measure $d A$ which appears also in the definition of $\mathcal{L}_{V}$ above. The measure $d A$ is on the group $\mathbb{A}_{E}^{\times, V} / \mathbb{A}^{\times, V}=\mathbf{S}\left(\mathbb{A}^{V}\right)$ (and on $E_{V}^{\times} / F_{V}^{\times}$), where $\mathbf{S}$ is the torus $\mathrm{R}_{E / F} \mathrm{GL}(1) / \mathrm{GL}(1)$. We write $\mathrm{R}_{E / F}$ for the functor of restriction of scalars from $E$ to $F$. This measure is described as follows. The differential form $\left(a_{2} d a_{1}-a_{1} d a_{2}\right) /\left(a_{1}^{2}-\theta a_{2}^{2}\right)$ on $\mathrm{R}_{E / F} \mathrm{GL}(1)$ is the inverse image of an invariant differential form $\omega$ on $\mathbf{S}$. Also $\mathbf{S}$ has convergence factors $c_{v}=\left(1-q_{v}^{-1}\right)^{-1}$ when $v$ is split nonarchimedean place, and $c_{v}=\left(1+q_{v}^{-1}\right)^{-1}$ when $v$ is unramified nonsplit nonarchimedean place, and $c_{v}=1$ otherwise. From the form $\omega$ and the convergence factors $\left(c_{v}\right)$ we get a Tamagawa measure $d A=|\omega|_{\mathbb{A}}$ on $\mathbf{S}(\mathbb{A})$.

If $A=\left(\begin{array}{cc}a_{1} & a_{2} \\ a_{2} \theta & a_{1}\end{array}\right) \in \mathrm{GL}\left(2, \mathbb{A}^{V}\right)$, we write $A=\left(\begin{array}{cc}t_{1} & * \\ 0 & t_{2}\end{array}\right) k$, with $k=k(A)$ in the standard maximal compact subgroup of $\operatorname{GL}\left(2, \mathbb{A}^{V}\right)$, and we put $a^{*}=t_{1} / t_{2}$. This is an element of $\mathbb{A}^{\times, V}$, uniquely determined up to multiplication by an element of $U^{V}$. If $\chi^{V}$ is a character of $\mathbb{A}^{\times, V} / F^{\times} U^{V}$, then $A \mapsto \chi^{V}\left(a^{*}\right)$ defines a function on $\mathbb{A}_{E}^{\times, V} / \mathbb{A}^{\times, V}$. We shall need below to compute the integral $\int_{\mathbb{A}_{E}^{\times, V} / \mathbb{A}^{\times, V}} \chi^{V}\left(a^{*}\right) d A$, which is equal to $\prod_{v} c_{v} \int_{S_{v}} \chi_{v}\left(a_{v}^{*}\right) d A_{v}$. In the local computations one can take $x=a_{1} / a_{2}$ as a coordinate. The integration is then over $F_{v}$, and $d A_{v}=d x /\left|x^{2}-\theta\right|_{v}$.

Denote by $\chi_{\theta}$ the quadratic character of $F^{\times} \backslash \mathbb{A}^{\times}$associated with the quadratic extension $E / F$. Its unramified components satisfy $\chi_{\theta, v}\left(\boldsymbol{\pi}_{v}\right)=1$ if $v$ is split in $E,=-1$ if $v$ stays prime in $E$. The global $L$-function $L(\chi, \zeta)$ is the product of local factors. At the places 
where $\chi$ is unramified, $L_{v}\left(\chi_{v}, \zeta\right)=\left(1-\chi_{v}\left(\boldsymbol{\pi}_{v}\right) q_{v}^{-\zeta}\right)^{-1}$. We write $L(\zeta)$ for $L(\chi, \zeta)$ when $\chi=1$. Note that $\mathbb{A}^{\times}=\mathbb{R}_{+}^{\times} \times \mathbb{A}^{1}, \mathbb{A}^{1}$ is the group of idèles of volume 1 , and $\mathbb{R}_{+}^{\times}$embeds in $\mathbb{A}^{\times}$as the group of idèles with equal positive archimedean parts, and 1 at the finite places.

Proposition 10.1. Let $\chi$ be a unitary character of $\mathbb{A}^{\times} / F^{\times} U \mathbb{R}_{+}^{\times}$, and put $\chi_{\zeta}(x)=\chi(x)|x|^{\zeta}$. Then the integral

$$
\mathcal{L}^{V}\left(\chi_{\zeta}\right)=\mathcal{L}^{V}(\chi, \zeta)=\int_{\mathbb{A}_{E}^{\times, V} / \mathbb{A}^{x, V}} \chi^{V}\left(a^{*}\right)\left|a^{*}\right|^{\zeta} d A
$$

converges absolutely for $\operatorname{Re}(\zeta)>1$, and has meromorphic continuation to $\operatorname{Re}(\zeta)>0$, which is holomorphic on $\operatorname{Re}(\zeta) \geq 1 / 2$, except at $\zeta=1$ when $\chi$ is 1 or $\chi_{\theta}$. Moreover, $\mathcal{L}^{V}\left(\chi, \frac{1}{2}+\zeta\right)$ is slowly increasing on $\zeta \in i \mathbb{R}$. Almost all factors in the product expansion of $\mathcal{L}^{V}(\chi, \zeta)$ coincide with those of $L^{V}(\chi, \zeta) L^{V}\left(\chi_{\theta} \chi, \zeta\right) / L^{V}\left(\chi^{2}, 2 \zeta\right)$.

Proof. Locally we have that $\left|a^{*}\right|_{v}$ is $\left|a_{1}^{2}-\theta a_{2}^{2}\right| / \max \left(\left|a_{1}\right|,\left|\theta a_{2}\right|\right)^{2}$ ( $v$ nonarchimedean),

$$
\left|a_{1}^{2}-\theta a_{2}^{2}\right| /\left(a_{1}^{2}+\theta^{2} a_{2}^{2}\right) \quad(v \text { real }), \quad\left(a_{1}^{2}-\theta a_{2}^{2}\right)\left(\bar{a}_{1}^{2}-\bar{\theta} \bar{a}_{2}^{2}\right) /\left(a_{1} \bar{a}_{1}+\theta \bar{\theta} a_{2} \bar{a}_{2}\right)^{2} \quad(v \text { complex}) .
$$

Put $\chi^{\prime}(t)=\chi(t)|t|^{\zeta}$. At almost all places $v$, if $\theta$ is not a square in $F_{v}, \chi_{v}^{\prime}\left(a_{v}^{*}\right)$ is identically 1 , and then $c_{v} \int_{S_{v}} d A_{v}=1=L\left(\chi_{v}, \zeta_{v}\right) L\left(\chi_{\theta, v} \chi_{v}, \zeta_{v}\right) / L\left(\chi_{v}^{2}, 2 \zeta_{v}\right)$. Here $S_{v}=E_{v}^{\times} / F_{v}^{\times}$. If $\theta$ is a square in $F_{v}$, suppose that $|\theta|_{v}=1$. Then $\chi_{v}^{\prime}\left(a_{v}^{*}\right)=1$ except when $\left|a_{1} / a_{2} \pm \theta^{1 / 2}\right|<1$. Then

$$
\begin{aligned}
& \int_{S_{v}} \chi_{v}^{\prime}\left(a_{v}^{*}\right) d A_{v}=\int_{|x|>1} \frac{d x}{\left|x^{2}\right|}+\int_{|x| \leq 1,\left|x \pm \theta^{1 / 2}\right|=1} d x \\
& +\int_{\left|x-\theta^{1 / 2}\right|<1}\left|x-\theta^{1 / 2}\right|^{\zeta-1} \chi\left(x-\theta^{1 / 2}\right) d x+\int_{\left|x+\theta^{1 / 2}\right|<1}\left|x+\theta^{1 / 2}\right|^{\zeta-1} \chi\left(x+\theta^{1 / 2}\right) d x \\
& =q^{-1}+\left(1-2 q^{-1}\right)+2 \int_{|x|<1}|x|^{\zeta-1} \chi(x) d x \\
& =\left(1-q^{-1}\right)\left(1-q^{-\zeta} \chi(\boldsymbol{\pi})\right)^{-1}\left(1+q^{-\zeta} \chi(\boldsymbol{\pi})\right)=c_{v}^{-1} L\left(\chi_{v}, \zeta\right)^{2} / L\left(\chi_{v}^{2}, 2 \zeta\right)
\end{aligned}
$$

if $\operatorname{Re}(\zeta)>0$. It is easy to see for all $v$ that $c_{v} \int_{S_{v}} \chi_{v}\left(a_{v}^{*}\right)\left|a_{v}\right|^{\zeta} d A_{v}$ is a holomorphic function of $\zeta$ on $\operatorname{Re}(\zeta)>0$, hence the proposition follows.

9. Minimal parabolic. Next we examine the contribution to the summation formula from the term of (9.2) associated with a character (of the Levi subgroup) of the minimal parabolic subgroup $\mathbf{P}_{1}=\mathbf{B}$. Again we are led to consider $\Lambda^{T} E(h, \rho, \zeta)$ and its integral over $\mathbf{Z}(\mathbb{A}) C_{\theta} \backslash \mathbf{C}_{\theta}(\mathbb{A})$. The truncated Eisenstein series is computed in [A2], Lemma 4.1:

$$
\begin{gathered}
\Lambda^{T} E(h, \Phi, \rho, \zeta) \\
=\sum_{s \in W} \varepsilon_{0}(s \zeta) \sum_{\gamma \in B \backslash G} \Phi_{0}\left(s \zeta, H_{0}(\gamma h)-T\right) e^{\left\langle s \zeta+\rho_{0}, H_{0}(\gamma h)\right\rangle}(M(s, \rho, \zeta) \Phi)(\gamma h) .
\end{gathered}
$$

The character $\rho=(\chi, \omega)$ of the diagonal subgroup is determined by the unitary characters $\chi, \omega$ of $\mathbb{A}^{\times} / F^{\times} \mathbb{R}_{+}^{\times}$, via $\rho(\operatorname{diag}(a, b, \lambda / b, \lambda / a))=\chi\left(a^{2} / \lambda\right) \omega(a b / \lambda)$. Let $\delta(\chi \mid U)$ be 1 if the restriction $\chi \mid U$ of $\chi$ to $U$ is 1 , and 0 if $\chi \mid U \neq 1$. Let $\delta(\chi)$ be 1 if $\chi$ is 1 , and 0 if $\chi \neq 1$. 
Proposition 10.2. Suppose that $\Phi$ is of the form $I\left(\rho, \zeta, f_{2}\right) \Phi^{\prime}$, where $f_{2} \in C_{c}^{\infty}(\mathbf{G}(\mathbb{A}))$ is a $\mathbb{K}$-finite function which is spherical outside $V$. Then $\Phi$ is (right) $\mathbb{K}$-finite and $\mathbb{K}^{V}$-invariant. Then the integral of $(1 *)$ over $\mathbf{Z}(\mathbb{A}) C_{\theta} \backslash \mathbf{C}_{\theta}(\mathbb{A})$ is the sum of

$$
\begin{gathered}
\delta(\chi \omega) \delta(\chi \mid U) \frac{t_{2}^{\zeta_{1}+\zeta_{2}-\frac{1}{2}}}{\zeta_{1}+\zeta_{2}-\frac{1}{2}} \mathcal{L}^{V}\left(\omega^{-1}, \frac{1}{2}+\zeta_{1}\right) \mathcal{L}_{V}\left(s_{1}, \rho, \zeta, \Phi\right), \\
\delta(\chi) \delta(\chi \omega \mid U) \frac{t_{2}^{\zeta_{1}-\frac{1}{2}}}{\zeta_{1}-\frac{1}{2}} \mathcal{L}^{V}\left(\omega, \frac{1}{2}+\zeta_{1}+\zeta_{2}\right) \mathcal{L}_{V}\left(s_{\alpha_{2}}, \rho, \zeta, \Phi\right) \\
-\delta(\chi \omega) \delta(\chi \mid U) \frac{t_{2}^{-\zeta_{1}-\zeta_{2}-\frac{1}{2}}}{\zeta_{1}+\zeta_{2}+\frac{1}{2}} \mathcal{L}^{V}\left(\omega^{-1}, \frac{1}{2}+\zeta_{1}\right) \mathcal{L}_{V}\left(s_{\alpha_{2}} s_{\alpha_{1}} s_{\alpha_{2}}, \rho, \zeta, \Phi\right) \\
-\delta(\chi) \delta(\chi \omega \mid U) \frac{t_{2}^{-\zeta_{1}-\frac{1}{2}}}{\zeta_{1}+\frac{1}{2}} \mathcal{L}^{V}\left(\omega, \frac{1}{2}+\zeta_{1}+\zeta_{2}\right) \mathcal{L}_{V}\left(s_{\alpha_{2}} s_{\alpha_{1}}, \rho, \zeta, \Phi\right)
\end{gathered}
$$

Remark. We may define $\mathcal{L}(s, \rho, \zeta, \Phi)$ by the same formula which defines $\mathcal{L}_{V}$, but with $\int_{E_{V}^{\times} / F_{V}^{\times}}$replaced by $\int_{\mathbb{A}_{E}^{\times} / \mathbb{A}^{\times}}$. Then $\mathcal{L}=\mathcal{L}_{V} \mathcal{L}^{V}$, where $\mathcal{L}^{V}$ is as displayed in the Proposition $\left(=\mathcal{L}^{V}\left(\omega^{-1}, \frac{1}{2}+\zeta_{1}\right)\right.$ for $s=s_{1}$, etc. $)$, independent of $\Phi$.

Proof. This takes most of this section. By Proposition 1(c) we have $G=B C_{\theta} \cup \gamma_{0} B^{\gamma_{0}} C_{\theta}$, where $B^{\gamma_{0}}=\gamma_{0}^{-1} B \gamma_{0}$. To integrate $(1 *)$ over $\mathbf{Z}(\mathbb{A}) C_{\theta} \backslash \mathbf{C}_{\theta}(\mathbb{A})$ we shall use the formula

$$
\begin{gathered}
\int_{\mathbf{Z}(\mathbb{A}) C_{\theta} \backslash \mathbf{C}_{\theta}(\mathbb{A})} \sum_{\gamma \in B \backslash G} \Phi^{\prime}(\gamma h) d h \\
=\int_{\mathbf{Z}(\mathbb{A}) \cdot B \cap C_{\theta} \backslash \mathbf{C}_{\theta}(\mathbb{A})} \Phi^{\prime}(h) d h+\int_{\mathbf{Z}(\mathbb{A}) \cdot B^{\gamma_{0}} \cap C_{\theta} \backslash \mathbf{C}_{\theta}(\mathbb{A})} \Phi^{\prime}\left(\gamma_{0} h\right) d h .
\end{gathered}
$$

To perform this integration, recall the root diagram for $\operatorname{Sp}(4)$ from section 2 , and the notations there, used in $(1 *)$. The weights can be identified with $\varpi_{1}=\alpha_{2}+2 \alpha_{1}=2 \varepsilon_{1}$ and $\varpi_{2}=\alpha_{1}+\alpha_{2}=\varepsilon_{1}+\varepsilon_{2}$. We have $H_{0}(\tilde{a} n k)=H_{0}(\tilde{a})$, and if $\tilde{a}=\operatorname{diag}(a, b, \lambda / b, \lambda / a)$, then

$$
H=H(\tilde{a})=H_{0}(\tilde{a})=\ln \left|\frac{a}{b}\right| \cdot \varpi_{1}+\ln \left|\frac{b^{2}}{\lambda}\right| \cdot \varpi_{2}=\ln \left|\frac{a^{2}}{\lambda}\right| \cdot \alpha_{1}+\ln \left|\frac{a b}{\lambda}\right| \cdot \alpha_{2} .
$$

The vector $\zeta$ has the form $\zeta=\zeta_{1} \cdot \varpi_{1}+\zeta_{2} \cdot \varpi_{2}$, both $\zeta_{1}$ and $\zeta_{2}$ are assumed to have large real parts for our computations. The following is a list of the 8 elements in the Weyl group $W$ (they will be denoted below by $s_{1}=1, \cdots, s_{8}$, according to the following tabulation), of $s \zeta, \varepsilon_{0}(s \zeta), \phi_{0}(s \zeta, H-T)$, and $e^{\langle s \zeta, H\rangle}$. Note that $\rho_{0}$ is half the sum of the positive roots, and $e^{\left\langle\rho_{0}, H\right\rangle}=\left|a^{4} b^{2} / \lambda^{3}\right|^{1 / 2}$. The dihedral group $W=D_{4}$ is generated by reflections $s_{\alpha_{1}}$ and $s_{\alpha_{2}}$ corresponding to the simple roots $\alpha_{1}$ and $\alpha_{2}\left(s_{\alpha_{i}}\left(\alpha_{i}\right)=-\alpha_{i}\right)$. Being a subgroup of the symmetric group $S_{4}$ on 4 letter, these elements can be represented by 
permutations: $s_{\alpha_{2}}=(23), s_{\alpha_{1}}=(12)(34), s_{\alpha_{2}} s_{\alpha_{1}} s_{\alpha_{2}}=(13)(24)$, etc. The longest element of $W$ is $w_{0}=s_{\alpha_{1}} s_{\alpha_{2}} s_{\alpha_{1}} s_{\alpha_{2}}=(14)(23)$, a $180^{\circ}$ rotation, while $s_{\alpha_{1}} s_{\alpha_{2}}=$ (3124) is an anti-clockwise rotation of $90^{\circ}$. Further, $T=\ln t_{1} \cdot \alpha_{1}+\ln t_{2} \cdot \alpha_{2}, t_{i}$ large positive numbers.

\begin{tabular}{|c|c|c|c|c|c|}
\hline$s$ & $s \zeta\left(\zeta_{i}>0\right)$ & $\varepsilon_{0}(s \zeta)$ & \multicolumn{2}{|c|}{$\phi_{0}(s \zeta, H-T)$} & $e^{\langle s \zeta, H\rangle}$ \\
\hline 1 & $\zeta_{1} \varpi_{1}+\zeta_{2} \varpi_{2}$ & + & $\left|\frac{a^{2}}{\lambda}\right|<$ & $\mid<t_{2}$ & $\left|\frac{a^{2}}{\lambda}\right| \zeta_{1}\left|\frac{a b}{\lambda}\right| \zeta_{2}$ \\
\hline$w_{0}=(14)(23)$ & $-\zeta_{1} \varpi_{1}-\zeta_{2} \varpi_{2}$ & + & $>$ & $>$ & $\left.|\cdot| \hat{\mid}^{-} \zeta_{1}|\cdot|\right|^{-\zeta_{2}}$ \\
\hline$s_{\alpha_{1}}=(12)(34)$ & $-\zeta_{1} \varpi_{1}+\left(2 \zeta_{1}+\zeta_{2}\right) \varpi_{2}$ & - & $>$ & $<$ & $|\cdot|^{-\zeta_{1}}|\cdot|^{2 \zeta_{1}+\zeta_{2}}$ \\
\hline$s_{\alpha_{2}} s_{\alpha_{1}} s_{\alpha_{2}}$ & $\zeta_{1} \varpi_{1}-\left(2 \zeta_{1}+\zeta_{2}\right) \varpi_{2}$ & - & $<$ & $>$ & $|\cdot| \zeta_{1}|\cdot|^{-2 \zeta_{1}-\zeta_{2}}$ \\
\hline$s_{\alpha_{1}} s_{\alpha_{2}} s_{\alpha_{1}}=(14)$ & $-\left(\zeta_{1}+\zeta_{2}\right) \varpi_{1}+\zeta_{2} \varpi_{2}$ & - & $>$ & $<$ & $|\cdot|-\zeta_{1}-\zeta_{2}|\cdot|_{2}$ \\
\hline$s_{\alpha_{2}}=(23)$ & $\left(\zeta_{1}+\zeta_{2}\right) \varpi_{1}-\zeta_{2} \varpi_{2}$ & - & $<$ & $>$ & $|\cdot| \zeta_{1}+\zeta_{2}|\cdot|^{-\zeta_{2}}$ \\
\hline$s_{\alpha_{1}} s_{\alpha_{2}}=(3124)$ & $-\left(\zeta_{1}+\zeta_{2}\right) \varpi_{1}+\left(2 \zeta_{1}+\zeta_{2}\right) \varpi_{2}$ & - & $>$ & $<$ & $\left.\cdot\left|-\zeta_{1}-\zeta_{2}\right| \cdot\right|^{2 \zeta_{1}+\zeta_{2}}$ \\
\hline$s_{\alpha_{2}} s_{\alpha_{1}}=(2134)$ & $\left(\zeta_{1}+\zeta_{2}\right) \varpi_{1}-\left(2 \zeta_{1}+\zeta_{2}\right) \varpi_{2}$ & - & $<$ & $>$ & $\left.\cdot\left|\zeta_{1}+\zeta_{2}\right| \cdot\right|^{-2 \zeta_{1}-\zeta_{2}}$ \\
\hline
\end{tabular}

Let us return now to the last integral in $(2 *)$. The intersection $B \cap C_{\theta}$ consist of $\left(\begin{array}{cc}a_{1} & b \\ 0 & d_{1}\end{array}\right)$, $a_{1}, d_{1}$ are scalars in $F^{\times}$and $b=\left(\begin{array}{cc}b_{1} & b_{2} \\ \theta b_{2} & b_{1}\end{array}\right), b_{1}, b_{2} \in F$. Also $B^{\gamma_{0}} \cap C_{\theta}$ is the group of $\left(\begin{array}{cc}a_{1} & b \\ 0 & d_{1}\end{array}\right)$ in $B \cap C_{\theta}$ with $b_{2}=0$. Hence we have $B \cap C_{\theta}=\left(B^{\gamma_{0}} \cap C_{\theta}\right) N_{2}$. Here $N_{2}$ is the group of $\left(\begin{array}{ll}I & b \\ 0 & I\end{array}\right)$ with $b=\left(\begin{array}{cc}0 & b_{2} \\ \theta b_{2} & 0\end{array}\right)$. Hence the last integral in $(2 *)$ is equal to

$$
\int_{\mathbf{Z}(\mathbb{A}) \cdot B \cap C_{\theta} \backslash \mathbf{C}_{\theta}(\mathbb{A})} d h \int_{\mathbf{N}_{2}(\mathbb{A})} \Phi^{\prime}\left(\gamma_{0} n h\right) d n .
$$

Lemma 10.3. (1) If $n=\left(\begin{array}{ll}I & b \\ 0 & I\end{array}\right)$ and $h=\left(\begin{array}{ll}I & 0 \\ 0 & \lambda\end{array}\right)\left(\begin{array}{cc}A & 0 \\ 0 & \varepsilon A \varepsilon\end{array}\right) k$, with $b=\left(\begin{array}{cc}b_{1} & b_{2} \\ b_{2} \theta & b_{1}\end{array}\right), b_{i} \in$ $\mathbb{A} / F ; \lambda \in F^{\times} \backslash \mathbb{A}^{\times} ; A=\left(\begin{array}{cc}a_{1} & a_{2} \\ a_{2} \theta & a_{1}\end{array}\right) \in \mathrm{GL}(2, \mathbb{A}) ; k \in \mathbb{K}_{\theta}$, and $a^{*}$ is defined from $A$ as in Proposition 10.1, then

$$
H_{0}\left(\gamma_{0} n h\right)=s_{\alpha_{2}} H_{0}(h)+H_{0}\left(\gamma_{0} n^{\prime}\right), \quad \text { where } \quad n^{\prime}=\left(\begin{array}{cc}
I & b^{\prime} \\
0 & I
\end{array}\right), b^{\prime}=\left(\begin{array}{cc}
0 & \lambda a^{*} b_{2} \\
\theta \lambda a^{*} b_{2} & 0
\end{array}\right) .
$$

(2) We have $e^{\left\langle\rho_{0}, \gamma_{0} H_{0}(h)\right\rangle}=e^{\left\langle\rho_{0}, H_{0}(h)\right\rangle}\left|\lambda a^{*}\right|$.

(3) The function $\phi_{0}\left(s \zeta, H_{0}\left(\gamma_{0} n h\right)-T\right)$ is independent of $n$. For a sufficiently large $t_{2}$ it is identically zero if $s=s_{2}, s_{4}, s_{6}, s_{8}$.

(4) If

$$
\Phi^{\prime}(h)=\phi_{0}\left(s \zeta, H_{0}(h)-T\right) e^{\left\langle s \zeta+\rho_{0}, H_{0}(h)\right\rangle}(M(s, \rho, \zeta) \Phi)(h),
$$

then

$$
\int_{\mathbf{N}_{2}(\mathbb{A})} \Phi^{\prime}\left(\gamma_{0} n h\right) d n=\phi_{0}\left(s \zeta, H_{0}\left(\gamma_{0} h\right)-T\right) e^{\left\langle s_{\alpha_{2}} s \zeta+\rho_{0}, H_{0}(h)\right\rangle}\left(M\left(s_{\alpha_{2}} s, \rho, \zeta\right) \Phi\right)(h) .
$$

Proof. (1) Write $h^{\prime} \equiv h^{\prime \prime}$ for $\mathbf{N}(\mathbb{A}) h^{\prime} \mathbb{K}=\mathbf{N}(\mathbb{A}) h^{\prime \prime} \mathbb{K}$. Then $h \equiv h_{0}, h_{0}=\operatorname{diag}\left(a^{*}, 1, \lambda a^{*}, \lambda\right)$, and $\gamma_{0} n h \equiv \gamma_{0} n h_{0} \equiv \gamma_{0} h_{0} n^{\prime}=\gamma_{0} h_{0} \gamma_{0}^{-1} \cdot \gamma_{0} n^{\prime}$. Note that $H_{0}\left(\gamma_{0} h\right)=s_{\alpha_{2}} H_{0}(h) ;(1)$ follows. 
(2) This follows from $s_{\alpha_{2}} \rho_{0}=\rho_{0}-\alpha_{2}$, and $e^{\left\langle-\alpha_{2}, H_{0}(h)\right\rangle}=\left|\lambda a^{*}\right|$.

(3) If $\gamma_{0} n h \equiv h_{0}^{\prime}$, with $h_{0}^{\prime}=\left(a_{0}^{\prime}, b_{0}^{\prime}, \lambda_{0}^{\prime} / b_{0}^{\prime}, \lambda_{0}^{\prime} / a_{0}^{\prime}\right)$, then $\phi_{0}\left(s \zeta, H_{0}\left(\gamma_{0} n h\right)-T\right)$ is 0 or 1 depending on whether $\left|a_{0}^{\prime 2} / \lambda_{0}^{\prime}\right|$ is bigger or smaller than $t_{1}$, and whether $\left|a_{0}^{\prime} b_{0}^{\prime} / \lambda_{0}^{\prime}\right|$ is bigger or smaller than $t_{2}$. Since $h_{0}^{\prime}=\gamma_{0} h_{0} \gamma_{0}^{-1} \cdot \gamma_{0} n^{\prime}$ (by the proof of (1)), the factor $\left|a_{0}^{\prime 2} / \lambda_{0}^{\prime}\right|=$ $\left|a^{*} / \lambda\right|$ is independent of $n$. Moreover, $\left|\lambda_{0}^{\prime}\right|=\left|\lambda a^{*}\right|$. Hence the factor $\left|a_{0}^{\prime} b_{0}^{\prime} / \lambda_{0}^{\prime}\right|$ is equal to

$$
\left|\frac{\lambda a^{* 2}}{\left(\theta a^{*} b_{2}, 1\right)} / \lambda a^{*}\right|=\left|a^{*} /\left(a^{*} \theta b_{2}, 1\right)\right| .
$$

If $x=\left(x_{v}\right), y=\left(y_{v}\right)$ in $\mathbb{A}^{\times}$, we put $(x, y)=\left(\left(x_{v}, y_{v}\right)\right) \in \mathbb{A}^{\times}$, where $\left(x_{v}, y_{v}\right)$ is an element of $F_{v}^{\times}$whose absolute value is $\left(x_{v}^{2}+y_{v}^{2}\right)^{1 / 2}$ if $v$ is real, $\left(x_{v} \bar{x}_{v}+y_{v} \bar{y}_{v}\right)^{1 / 2}$ if $v$ is complex, and $\max \left(\left|x_{v}\right|_{v},\left|y_{v}\right|_{v}\right)$ if $v$ is finite. But $\left|a^{*}\right|$ is bounded. Hence so is $\left|a_{0}^{\prime} b_{0}^{\prime} / \lambda_{0}^{\prime}\right|$. If $t_{2}$ is sufficiently large, the condition $\left|a_{0}^{\prime} b_{0}^{\prime} / \lambda_{0}^{\prime}\right|<t_{2}$ is always satisfied, but $\left|a_{0}^{\prime} b_{0}^{\prime} / \lambda_{0}^{\prime}\right|>t_{2}$ is never satisfied; (3) follows.

(4) This follows from (3), (1), the definition of the intertwining operator, and the functional equation for these operators:

$$
\begin{aligned}
& \int_{\mathbf{N}(\mathbb{A})_{2}} e^{\left\langle s \zeta+\rho_{0}, H_{0}\left(\gamma_{0} n h\right)\right\rangle}(M(s, \rho, \zeta) \Phi)\left(\gamma_{0} n h\right) d n \\
& =\left(M\left(s_{\alpha_{2}}, s \rho, s \zeta\right) M(s, \rho, \zeta) \Phi\right)(h)=\left(M\left(s_{\alpha_{2}} s, \rho, \zeta\right) \Phi\right)(h) .
\end{aligned}
$$

We conclude that the integral over $\mathbf{Z}(\mathbb{A}) C_{\theta} \backslash \mathbf{C}_{\theta}(\mathbb{A})$ of $(1 *)$ is equal to the integral over $\mathbf{Z}(\mathbb{A}) \cdot B \cap C_{\theta} \backslash \mathbf{C}_{\theta}(\mathbb{A})$ of the sum over $s \in W$ of the product of

$$
\varepsilon_{0}(s \zeta) \phi_{0}\left(s \zeta, H_{0}(h)-T\right)+\varepsilon_{0}\left(s_{6} s \zeta\right) \phi_{0}\left(s_{6} s \zeta, H_{0}\left(\gamma_{0} h\right)-T\right)
$$

with

$$
e^{\left\langle s \zeta+\rho_{0}, H_{0}(h)\right\rangle}(M(s, \rho, \zeta) \Phi)(h) .
$$

If $h=\operatorname{diag}\left(a^{*}, 1, \lambda a^{*}, \lambda\right)$, then $\gamma_{0} h \gamma_{0}^{-1}=\operatorname{diag}\left(a^{*}, \lambda a^{*}, 1, \lambda\right)$. Note that $\left|a(h)^{2} / \lambda(h)\right|=$ $\left|a^{*} / \lambda\right|,|a(h) b(h) / \lambda(h)|=|1 / \lambda|$, and the corresponding quantities for $\gamma_{0} h \gamma_{0}^{-1}$ are $\left|a^{*} / \lambda\right|$ and $\left|a^{*}\right|$. As noted in Lemma 10.3(3), the function $\phi_{0}\left(s_{6} s \zeta, H_{0}\left(\gamma_{0} h\right)-T\right)$ is 0 for $s=s_{1}, s_{3}, s_{5}, s_{7}$, and by the table above, it is the characteristic function of $\left|a^{*} / \lambda\right|>t_{1}$ for $s=s_{2}, s_{4}, s_{8}$, and of $\left|a^{*} / \lambda\right|<t_{1}$ for $s=s_{6}$. This function appears in $(3 *)_{s}$ multiplied by $\varepsilon_{0}\left(s_{6} s \zeta\right)$, which is 1 for $s=s_{6}$ and -1 otherwise.

To compute $\phi_{0}\left(s \zeta, H_{0}(h)-T\right)$, let us restrict attention to $t_{1}, t_{2}$ with $t_{1}>c t_{2}$, where $c$ is a constant such that $\left|a^{*}\right| \leq c$. Then $\left|a^{*} / \lambda\right|>t_{1}$ implies $|1 / \lambda|>t_{2}$, and $|1 / \lambda|<t_{2}$ implies $\left|a^{*} / \lambda\right|<t_{1}$. Hence $\phi_{0}\left(s \zeta, H_{0}(h)-T\right)$ is 0 for $s=s_{3}, s_{5}, s_{7}$. It is the characteristic function of $|1 / \lambda|<t_{2}$ for $s=s_{1}$, of $\left|a^{*} / \lambda\right|>t_{1}$ for $s=s_{2}$, and of $\left|a^{*} / \lambda\right|<t_{1},|1 / \lambda|>t_{2}$, when $s=s_{4}, s_{6}, s_{8}$. Since $\varepsilon_{0}(s \zeta)$ is 1 for $s=s_{1}, s_{2}$, and -1 otherwise, we conclude that $(3 *)_{s}$ is zero for $s=s_{2}, s_{3}, s_{5}, s_{7}$, it is the characteristic function of $|1 / \lambda|<t_{2}$ for $s=s_{1}, s_{6}$, and minus the characteristic function of $|1 / \lambda|>t_{2}$ for $s=s_{4}, s_{8}$.

The factor $e^{\left\langle\rho_{0}, H_{0}(h)\right\rangle}$ is $\left|a^{*} / \lambda\right|^{1 / 2}|1 / \lambda|$. The factors $e^{\left\langle s \zeta, H_{0}(h)\right\rangle}$ are computed using the table to be $\left|a^{*} / \lambda\right|^{\zeta_{1}}|1 / \lambda|^{\zeta_{2}}$ if $s=s_{1},\left|a^{*} / \lambda\right|^{\zeta_{1}+\zeta_{2}}|1 / \lambda|^{-\zeta_{2}}$ if $s=s_{6},\left|a^{*} / \lambda\right|^{\zeta_{1}}|1 / \lambda|^{-2 \zeta_{1}-\zeta_{2}}$ 
if $s=s_{4}$, and $\left|a^{*} / \lambda\right|^{\zeta_{1}+\zeta_{2}}|1 / \lambda|^{-2 \zeta_{1}-\zeta_{2}}$ if $s=s_{8}$. To perform the integration we use the Iwasawa decomposition

$$
h=\left(\begin{array}{ll}
I & b \\
0 & I
\end{array}\right)\left(\begin{array}{cc}
I & 0 \\
0 & \lambda
\end{array}\right)\left(\begin{array}{cc}
A & 0 \\
0 & \varepsilon A \varepsilon
\end{array}\right) k, \quad d h=|\lambda|^{2} d b d^{\times} \lambda d A d k .
$$

The four integrals are

$$
\begin{aligned}
& \int_{|\lambda|>1 / t_{2}}|\lambda|^{-\zeta_{1}-\zeta_{2}+\frac{1}{2}}(\chi \omega)^{-1}(\lambda) d^{\times} \lambda \cdot \iint\left|a^{*}\right|^{\zeta_{1}+\frac{1}{2}} \chi\left(a^{*}\right) d A \cdot\left(M\left(s_{1}, \rho, \zeta\right) \Phi\right)(d(A) k) d k, \\
& \int_{|\lambda|>1 / t_{2}}|\lambda|^{-\zeta_{1}+\frac{1}{2}} \chi^{-1}(\lambda) d^{\times} \lambda \cdot \int\left|a^{*}\right|^{\zeta_{1}+\zeta_{2}+\frac{1}{2}}(\chi \omega)\left(a^{*}\right) d A \cdot\left(M\left(s_{6}, \rho, \zeta\right) \Phi\right)(d(A) k) d k, \\
& -\int_{|\lambda|<1 / t_{2}}|\lambda|^{\zeta_{1}+\zeta_{2}+\frac{1}{2}}(\chi \omega)(\lambda) d^{\times} \lambda \cdot \int\left|a^{*}\right|^{\zeta_{1}+\frac{1}{2}} \chi\left(a^{*}\right) d A \cdot\left(M\left(s_{4}, \rho, \zeta\right) \Phi\right)(d(A) k) d k,
\end{aligned}
$$

and

$$
-\int_{|\lambda|<1 / t_{2}}|\lambda|^{\zeta_{1}+\frac{1}{2}} \chi(\lambda) d^{\times} \lambda \cdot \int\left|a^{*}\right|^{\zeta_{1}+\zeta_{2}+\frac{1}{2}}(\chi \omega)\left(a^{*}\right) d A \cdot\left(M\left(s_{8}, \rho, \zeta\right) \Phi\right)(d(A) k) d k .
$$

By Proposition 10.1 these are equal to the four integrals of Proposition 10.2, whose proof is now complete.

To examine the contribution of the term associated with $P_{1}=B$ to our summation formula, we replace the last integral in (9.2) by the complex conjugates of the four terms computed in Proposition 10.2. Note that Proposition 10.2 is proven under the assumption that $\operatorname{Re}\left(\zeta_{i}\right)$ are large, but by analytic continuation its result holds also when $\operatorname{Re}\left(\zeta_{i}\right)=0$. Now consider each of the four sums majorizing the sums obtained on inserting the four functions derived in Proposition 10.2 into (9.2), e.g.:

$$
A(\rho, \zeta)=\sum_{\rho} \sum_{\Phi}\left|E_{\theta}\left(I\left(f_{1}\right) \Phi, \rho, \zeta\right) \mathcal{L}^{V}\left(\omega^{-1}, \frac{1}{2}+\zeta_{1}\right) \mathcal{L}_{V}\left(s_{1}, \rho, \zeta, \Phi\right)\right| .
$$

We claim that the function $A(\rho, \zeta)$ is a Schwartz function on the imaginary plane $i \mathfrak{A}_{B}^{*}$. Indeed, by Proposition 9.3(3), for a given $f_{1}$ with a fixed $\mathbb{K}$-type, $\left|E_{\theta}\left(I\left(f_{1}\right) \Phi, \rho, \zeta\right)\right|$ is bounded by some $C(\rho)(1+\|\zeta\|)^{c(\rho)}$. By Proposition 10.1, this bound holds also for $\mathcal{L}^{V}$. The sum over $\Phi$ is finite, it ranges only over vectors with the given $\mathbb{K}$-type. Moreover,

$$
\left|\int_{\mathbb{K}_{\theta}}\left(M(s, \rho, \zeta) I\left(\rho, \zeta ; f_{2}\right) \Phi\right)(k) d k\right| \leq\left\|I\left(\rho, \zeta ; f_{2}\right)\right\|,
$$

where the last norm is the operator norm on the finite dimensional space of vectors with a given $\mathbb{K}$-type. This norm is bounded by the norm of some matrix of the form

$$
\left(\int_{\mathbf{A}(\mathbb{A}) \mathbf{U}(\mathbb{A})} f_{2}\left(k_{i}^{-1} a u k_{j}\right) d u \cdot \rho(a) \delta(a)^{\zeta} d a\right), \quad\left(k_{i} \in \mathbb{K}\right) .
$$


It follows that the function $A(\rho, \zeta)$ is a Schwartz function as claimed, hence its integral on the imaginary plane $i \mathfrak{A}_{B}^{*}$ is finite. By the last assertion of Proposition 9, that $\iint K_{f, \chi}(u, h) \bar{\psi}_{\theta}(u) d u d h$ is not affected by $\Lambda^{T}$, we may take the limit as $T$ goes to infinity. The limit as $t_{2} \rightarrow \infty$ of the product of $t_{2}^{-1 / 2}$ and a function which is uniformly bounded in $t_{2}$, is zero. Hence the terms associated to $P_{1}=B$ in (9.2) do not contribute to our summation formula.

10. One dimensional. Next we consider the possible contribution from the non cuspidal discrete spectrum, namely one-dimensional, representations on the maximal parabolics $\mathbf{P}(\mathbb{A})$ and $\mathbf{Q}(\mathbb{A})$.

Consider first such a representation on $\mathbf{P}(\mathbb{A})$. As noted in section $6, I_{P}\left(\rho, \zeta_{2}, \omega\right)=$ $I_{B}\left(\zeta_{1}, \zeta_{2}-\zeta_{1}, \chi, \omega / \chi\right)$ if $\rho=I\left(\zeta_{1},-\zeta_{1}\right) \otimes \chi$. Thus the contribution to (9.2) is obtained on replacing the first integral in (9.2) by one over $\zeta \in i \mathbb{R}$, and the last integral in (9.2) by the complex conjugate of the limit as $\varepsilon \rightarrow 0$ of the product of $\varepsilon$ and the sum of the four terms computed in Proposition 10.2, in which $\left(\zeta_{1}, \zeta_{2}\right)$ is replaced by $\left(\frac{1}{2}+\varepsilon, \zeta-\frac{1}{2}-\varepsilon\right)$. With this replacement, these four terms, multiplied by $\varepsilon$, are the following.

The term indexed by $s=1$ (at $\left(\zeta_{1}, \zeta_{2}\right)=\left(\frac{1}{2}+\varepsilon, \zeta-\frac{1}{2}-\varepsilon\right)$, multiplied by $\varepsilon$, and taking $\varepsilon \rightarrow 0)$ is

$$
\delta(\chi \omega) \delta(\chi \mid U) \frac{t_{2}^{\zeta-\frac{1}{2}}}{\zeta-\frac{1}{2}} \cdot \lim _{\varepsilon \rightarrow 0} \varepsilon \mathcal{L}^{V}(\chi, 1+\varepsilon) \cdot \mathcal{L}_{V}\left(s_{1}, \rho, \zeta, \Phi\right) .
$$

The limit at $\varepsilon=0$ exists since $\mathcal{L}^{V}(\chi, 1+\varepsilon)$ has at most a simple pole at $\varepsilon=0$. As $t_{2} \rightarrow \infty$, the factor $t_{2}^{-1 / 2}$ dominates and no contribution to (9.2) is made.

The term indexed by $s=s_{4}$ is

$$
-\delta(\chi \omega) \delta(\chi \mid U) \frac{t_{2}^{-\zeta-\frac{1}{2}}}{\zeta+\frac{1}{2}} \cdot \lim _{\varepsilon \rightarrow 0} \varepsilon \mathcal{L}^{V}(\chi, 1+\varepsilon) \cdot \mathcal{L}_{V}\left(s_{4}, \rho, \zeta, \Phi\right) ;
$$

it makes no contribution to (9.2) either, as $t_{2}^{-1 / 2} \rightarrow 0$ when $t_{2} \rightarrow \infty$. The same conclusion holds for the term indexed by $s=s_{8}$, where the term is

$$
-\delta(\chi) \delta(\chi \omega \mid U) \frac{t_{2}^{-1-\varepsilon}}{1+\varepsilon} \mathcal{L}^{V}\left(\omega, \frac{1}{2}+\zeta\right) \cdot \mathcal{L}_{V}\left(s_{8}, \rho, \zeta, \Phi\right),
$$

and its product by $\varepsilon$ has the limit 0 as $\varepsilon \rightarrow 0$.

However, the term indexed by $s=s_{6}=s_{\alpha_{2}}=(23)$ is zero, unless $\omega$ is a character of $\mathbb{A}^{\times} / F^{\times} U \mathbb{R}_{+}^{\times}$, in which case it takes the form

$$
\delta(\chi) \delta(\chi \omega \mid U) \varepsilon^{-1} t_{2}^{\varepsilon} \mathcal{L}^{V}\left(\omega, \frac{1}{2}+\zeta\right) \cdot \mathcal{L}_{V}\left(s_{6},(1, \omega),\left(\frac{1}{2}+\varepsilon, \zeta-\frac{1}{2}-\varepsilon\right), \Phi\right) .
$$

The limit as $\varepsilon \rightarrow 0$ of the product of this with $\varepsilon$, is

$$
\mathcal{L}^{V}\left(\omega, \frac{1}{2}+\zeta\right) \cdot \mathcal{L}_{V}\left(s_{6},(1, \omega),\left(\frac{1}{2}, \zeta-\frac{1}{2}\right), \Phi\right) .
$$


The complex conjugate of this, inserted in (9.2) instead of the last integral in (9.2), makes the contribution (10.1) to our summation formula.

Next we consider the possible contributions to the summation formula associated to the one dimensional representations of the parabolic subgroup $\mathbf{Q}(\mathbb{A})$. As noted in section 6 , we have that $I_{Q}\left(\rho, \zeta_{3}\right)=I_{B}\left(\zeta_{3}-\zeta_{4}, 2 \zeta_{4}, \chi^{-1}, 1\right)$, if $\rho=I\left(\zeta_{4},-\zeta_{4}\right) \otimes \chi$. The one dimensional representations of $\operatorname{GL}(2, \mathbb{A})$ are obtained as the quotient of $\rho$ as $\zeta_{4} \rightarrow \frac{1}{2}$. Thus we need to consider the four terms of Proposition 10.2, in which $\left(\zeta_{1}, \zeta_{2}\right)$ is replaced by $\left(\zeta-\frac{1}{2}-\varepsilon, 1+2 \varepsilon\right)$, multiply by $\varepsilon$, take the limit as $\varepsilon \rightarrow 0$, substitute the complex conjugate of the result for the second integral in (9.2), replace the first integral in (9.2) by one over $\zeta$ in $i \mathbb{R}$, and take the limit as $t_{2} \rightarrow \infty$. We will see that this limit is 0 in all cases, hence no contribution is made to our summation formula.

The computations are as follows. The term indexed by $s=1$, multiplied by $\varepsilon$, is

$$
\frac{t_{2}^{\zeta+\varepsilon}}{\zeta+\varepsilon} \cdot \varepsilon \cdot \mathcal{L}^{V}(\chi, \zeta-\varepsilon) \cdot \mathcal{L}_{V}\left(s_{1}, \rho, \zeta, \Phi\right)
$$

At any $\zeta \neq 0$, the limit as $\varepsilon \rightarrow 0$ is zero. Similarly, the term indexed by $s=s_{8}$ is

$$
-\frac{t_{2}^{-\zeta+\varepsilon}}{\zeta-\varepsilon} \cdot \varepsilon \cdot \mathcal{L}^{V}(\omega, 1+\zeta-\varepsilon) \cdot \mathcal{L}_{V}\left(s_{8}, \rho, \zeta, \Phi\right),
$$

and its limit as $\varepsilon \rightarrow 0$ is zero for any $\zeta \neq 0$. The term indexed by $s=s_{4}$ is

$$
-\frac{t_{2}^{-1-\zeta-\varepsilon}}{1+\zeta+\varepsilon} \cdot \varepsilon \cdot \mathcal{L}^{V}(\chi, \zeta-\varepsilon) \cdot \mathcal{L}_{V}\left(s_{4}, \rho, \zeta, \Phi\right)
$$

and that indexed by $s=s_{6}$ is

$$
-\frac{t_{2}^{-1+\zeta-\varepsilon}}{1-\zeta+\varepsilon} \cdot \varepsilon \cdot \mathcal{L}^{V}(\omega, 1+\zeta+\varepsilon) \cdot \mathcal{L}_{V}\left(s_{6}, \rho, \zeta, \Phi\right) .
$$

Both have the limit 0 as $\varepsilon \rightarrow 0$, when $\zeta \neq 0$.

11. Maximal parabolics. Consider next the case of the Siegel parabolic $P$ where $\rho$ is a cuspidal representation of the Levi subgroup $\mathbf{M}(\mathbb{A})$ of $\mathbf{P}(\mathbb{A})$, whose restriction to $\mathbf{Z}(\mathbb{A})$ is trivial. The function $\Phi: \mathbf{Z}(\mathbb{A}) \mathbf{N}(\mathbb{A}) M \backslash \mathbf{G}(\mathbb{A}) \rightarrow \mathbb{C}$ is smooth, has the property that $\int_{\mathbb{K}} \int_{\mathbf{Z}(\mathbb{A}) M \backslash \mathbf{M}(\mathbb{A})}|\Phi(m k)|^{2} d m d k$ is finite, and that for every $g \in \mathbf{G}(\mathbb{A})$, the function $m \mapsto$ $\Phi(m g)$ on $\mathbf{M}(\mathbb{A})$ is a cusp form in the space of $\rho$. Put $\Phi_{2}=I\left(f_{2}, \rho, \zeta\right) \Phi$. Then in our case

$$
\begin{aligned}
\Lambda^{T} E\left(h, \Phi_{2}, \rho, \zeta\right) & =\sum_{\gamma \in P \backslash G} \Phi_{2}(\gamma h) \delta_{P}(\gamma h)^{\frac{1}{2}+\frac{\zeta}{3}} \chi\left(\delta_{P}(\gamma h)<t^{3}\right) \\
& -\sum_{\gamma \in P \backslash G}\left(M \Phi_{2}\right)(\gamma h) \delta_{P}(\gamma h)^{\frac{1}{2}-\frac{\zeta}{3}} \chi\left(\delta_{P}(\gamma h)>t^{3}\right) .
\end{aligned}
$$


Here $T=t^{3}$ is a large positive number, $\zeta$ is a complex number with a large real part, $\delta_{P}(g)$ is the modular function defined by $\delta_{P}(g)=|\operatorname{det}(\operatorname{Ad}(p) \mid \operatorname{LieN})|$ if $g=p k(p \in \mathbf{P}(\mathbb{A}), k \in$ $\left.\mathbb{K} ; \delta_{P}\left(\operatorname{diag}\left(A, \lambda w^{t} A^{-1} w\right)\right)=|| A|/ \lambda|^{3}\right), \chi(X)$ is the characteristic function defined by the condition $X$, and $M \Phi_{2}$ is the image of $\Phi_{2}$ under the action of the standard intertwining operator. The exponent of $\delta_{P}$ is taken to be $\zeta / 3$ to be consistent with our parametrization of induced representations.

To integrate $\Lambda^{T} E$ over $\mathbf{Z}(\mathbb{A}) C_{\theta} \backslash \mathbf{C}_{\theta}(\mathbb{A})$, recall from Proposition $1(\mathrm{c})$ that $G=P C_{\theta} \cup$ $P \gamma_{0} C_{\theta}$, hence that

$$
\begin{gathered}
\int_{\mathbf{Z}(\mathbb{A}) C_{\theta} \backslash \mathbf{C}_{\theta}(\mathbb{A})} \sum_{\gamma \in P \backslash G} \Phi^{\prime}(\gamma h) d h=\int_{\mathbf{Z}(\mathbb{A}) \cdot P \cap C_{\theta} \backslash \mathbf{C}_{\theta}(\mathbb{A})} \Phi^{\prime}(h) d h \\
+\int_{\mathbf{Z}(\mathbb{A}) \cdot P_{0} \backslash \mathbf{P}(\mathbb{A})_{0}} d p \int_{\mathbf{P}(\mathbb{A})_{0} \backslash \gamma_{0} \mathbf{C}_{\theta}(\mathbb{A}) \gamma_{0}^{-1}} \Phi^{\prime}\left(p h \gamma_{0}\right) d h,
\end{gathered}
$$

where $\mathbf{P}_{0}=\mathbf{P} \cap \gamma_{0} \mathbf{C}_{\theta} \gamma_{0}^{-1}$.

To compute the first integral on the right of $(5 *)$, note that $\mathbf{C}_{\theta}(\mathbb{A})=\mathrm{GL}\left(2, \mathbb{A}_{E}\right)^{\prime}$, the prime indicating that the determinant lies in $\mathbb{A}^{\times}$, and use the Iwasawa decomposition $h=$ $n a\left(\begin{array}{ll}I & 0 \\ 0 & \lambda\end{array}\right) k, d h=\left|\lambda^{2}\right| d n d a d^{\times} \lambda d k$ if $a \in \mathbf{T}(\mathbb{A})=\left\{d(A)=\operatorname{diag}(A, \varepsilon A \varepsilon) ; A=\left(\begin{array}{cc}a_{1} & a_{2} \\ a_{2} \theta & a_{1}\end{array}\right) \in\right.$ $\left.\mathbb{A}_{E}^{\times}\right\}$. Put $\Phi_{2}^{\mathbb{K}_{\theta}}(x)=\int_{\mathbb{K}_{\theta}} \Phi_{2}(x k) d k$. If $\Phi^{\prime}(x)=\Phi_{2}(x) \delta_{P}(x)^{\frac{1}{2}+\frac{\zeta}{3}} \chi\left(\delta_{P}(x)<t^{3}\right)$, then the integral of $\Phi^{\prime}$ on $\mathbf{Z}(\mathbb{A}) \cdot P \cap C_{\theta} \backslash \mathbf{C}_{\theta}(\mathbb{A})$ is equal to the product of $\left|\mathbb{A}_{E} / E\right|(=1$, this factor is obtained from the integral over $\left.N \cap C_{\theta} \backslash \mathbf{N}(\mathbb{A}) \cap \mathbf{C}_{\theta}(\mathbb{A})\right), \mathcal{L}\left(\Phi_{2}^{\mathbb{K}_{\theta}}\right)=\int_{\mathbf{Z}(\mathbb{A}) T \backslash \mathbf{T}(\mathbb{A})} \Phi_{2}^{\mathbb{K}_{\theta}}(a) d a$, and

$$
\int_{\left|\lambda^{-1}\right| \leq t}|\lambda|^{-\frac{3}{2}-\zeta}\left|\lambda^{-2}\right|^{-1} d^{\times} \lambda=\int_{|\lambda|<t}|\lambda|^{\zeta-\frac{1}{2}} d^{\times} \lambda=\frac{t^{\zeta-\frac{1}{2}}}{\zeta-\frac{1}{2}} .
$$

The integral of the last factor converges since $\operatorname{Re}(\zeta)>\frac{1}{2}$. The factor $\mathcal{L}\left(\Phi_{2}^{\mathbb{K} \theta}\right)$ is the integral of a cusp form, $\Phi_{2}^{\mathbb{K}_{\theta}}$, in $\rho$, a cuspidal representation of the group $d(\operatorname{PGL}(2, \mathbb{A})) \simeq$ $\operatorname{PGL}(2, \mathbb{A})$, over the homogeneous space $\mathbf{Z}(\mathbb{A}) T \backslash \mathbf{T}(\mathbb{A})$. According to a well-known result of Waldspurger [Wa1/2], see also Jacquet [J1] for a proof similar to the one of this paper, such an integral is equal to a product of the values at $\zeta=\frac{1}{2}$ of the $L$-functions $L(\rho, \zeta)$ and $L\left(\rho \otimes \chi_{\theta}, \zeta\right)$ (both depending on the cusp form $\Phi_{2}{ }^{K_{\theta}}$ ) attached to the cuspidal modules $\rho$ and $\rho \otimes \chi_{\theta}$, where $\chi_{\theta} \neq 1$ is the quadratic character of $F^{\times} \backslash \mathbb{A}^{\times}$associated with the quadratic extension $E / F$.

By analytic continuation, the computation of the part of $(5 *)$ under discussion holds for all complex $\zeta$, in particular for $\zeta$ in $i \mathfrak{A}_{P}^{*}=i \mathbb{R}$. These are the $\zeta$ which appear in (9.2). In fact it is the complex conjugate of $(4 *)$ which appears in $(9.2)$, thus we need to replace $\zeta$ by $-\zeta$ in our formula. The corresponding part of $(9.2)$ then takes the form

$$
t^{-\frac{1}{2}} n\left(A_{P}\right)^{-1} \sum_{\rho} \int_{i \mathbb{R}} \frac{t^{-\zeta}}{-\zeta-\frac{1}{2}} \sum_{\Phi}\left[E_{\theta}\left(I\left(f_{1}, \rho, \zeta\right) \Phi, \rho, \zeta\right) \overline{\mathcal{L}}\left(\Phi_{2}^{\mathbb{K}_{\theta}}\right)\right] d \zeta
$$


The function $\sum_{\rho} \sum_{\Phi}|[\cdots]|$ can be shown to have rapid decay in $\zeta \in i \mathbb{R}$, following the arguments of section 9 , using the $\mathbb{K}$-finiteness properties of $f_{1}$ and $f_{2}$, standard (polynomial) growth estimates on $L$-functions (same proof, based on the Phragmen-Lindelöf theorem, as that underlying Lemma 9.1), and a suitable analogue of Proposition 9.3(c). The limit as $t \rightarrow \infty$ of the product of $t^{-\frac{1}{2}}$ and a constant is clearly 0 .

The second term on the right side of $(4 *)$ contributes to the first integral on the right of $(5 *)$ a similar expression, but now $\Phi^{\prime}(x)=-\left(M \Phi_{2}\right)(x) \delta_{P}(x)^{\frac{1}{2}-\frac{\zeta}{3}} \chi\left(\delta_{P}(x)>t^{3}\right)$. The same Iwasawa decomposition shows that the integral of this $\Phi^{\prime}$ over $\mathbf{Z}(\mathbb{A}) \cdot P \cap C_{\theta} \backslash \mathbf{C}_{\theta}(\mathbb{A})$ is again the product $\mathcal{L}\left(\left(M \Phi_{2}\right)^{\mathbb{K}_{\theta}}\right)$ of values at $\zeta=\frac{1}{2}$ of $L(\rho, \zeta)$ and $L\left(\rho \otimes \chi_{\theta}, \zeta\right)$ (both $L$-functions depend on $\left.M \Phi_{2}\right)$, and of

$$
\int_{\left|\lambda^{-1}\right|>t}\left|\lambda^{-3}\right|^{\frac{1}{2}-\frac{\zeta}{3}}\left|\lambda^{-2}\right|^{-1} d^{\times} \lambda=\int_{|\lambda|<t^{-1}}|\lambda|^{\frac{1}{2}+\zeta} d^{\times} \lambda=\frac{t^{-\frac{1}{2}-\zeta}}{\frac{1}{2}+\zeta} \quad(\operatorname{Re} \zeta \geq 0) .
$$

Substituting this into (9.2) and noting that $\zeta \in i \mathbb{R}$, since $t^{-1 / 2} \rightarrow 0$ as $t \rightarrow \infty$, no contribution is made to the summation formula.

To study the possible contribution to the summation formula from any of the two terms on the right of $(4 *)$ to the last integral in $(5 *)$, note that $\gamma_{0} \mathbf{C}_{\theta} \gamma_{0}^{-1}=\left\{\left(\begin{array}{cc}A & B \varepsilon \\ \theta \varepsilon B & \varepsilon A \varepsilon\end{array}\right)\right\}$, and its intersection with $\mathbf{P}$ is $\mathbf{P}_{0}=\{\operatorname{diag}(A, \varepsilon A \varepsilon) ; A \in \mathrm{GL}(2)\}$. Since

$$
p \mapsto \int_{\mathbf{P}_{0}(\mathbb{A}) \backslash \gamma_{0} \mathbf{C}_{\theta}(\mathbb{A}) \gamma_{0}^{-1}} \Phi^{\prime}\left(p h \gamma_{0}\right) d h
$$

is a cusp form on $\mathbf{P}_{0}(\mathbb{A}) \simeq \mathrm{GL}(2, \mathbb{A})$, its integral over $\mathbf{Z}(\mathbb{A}) P_{0} \backslash \mathbf{P}_{0}(\mathbb{A})$ is 0 . Indeed, any cusp form on $\mathrm{GL}(2, \mathbb{A})$ is orthogonal to the constant functions.

In conclusion, the cuspidal representations $\rho$ of the Siegel parabolic $\mathbf{P}(\mathbb{A})$ make no contribution to the summation formula. The contribution from the other discrete spectrum representations $\rho$ of $\mathbf{P}(\mathbb{A})$, namely the one dimensional ones, has been discussed in the previous section.

The contribution to (9.2) from a cuspidal $\rho$ on $\mathbf{P}_{1}=\mathbf{Q}$ involves an integral over $\mathbf{Z}(\mathbb{A}) C_{\theta} \backslash \mathbf{C}_{\theta}(\mathbb{A})$ of an expression such as $(4 *)$, in which $P$ is replaced by $Q$. Since $\gamma_{0}$ lies in $Q$, we have $G=B C_{\theta} \cup B \gamma_{0} C_{\theta}=Q C_{\theta}$, and $Q \backslash G=Q \cap C_{\theta} \backslash C_{\theta}$. The intersection $Q \cap C_{\theta}$ consist of $\left(\begin{array}{cc}a_{1} & b \\ 0 & d_{1}\end{array}\right), a_{1}, d_{1}$ are scalars in $F^{\times}$and $b=\left(\begin{array}{cc}b_{1} & b_{2} \\ \theta b_{2} & b_{1}\end{array}\right), b_{1}, b_{2} \in F$. In particular the combined sum-integral over $Q \backslash G \times \mathbf{Z}(\mathbb{A}) C_{\theta} \backslash \mathbf{C}_{\theta}(\mathbb{A})=\mathbf{Z}(\mathbb{A}) \cdot Q \cap C_{\theta} \backslash \mathbf{C}_{\theta}(\mathbb{A})$ of the cusp form $\Phi$ on $\mathbf{M}(\mathbb{A})_{Q}$ factorizes. One of the integrals in the factorization will range over $N_{2} \backslash \mathbf{N}_{2}(\mathbb{A})$, where $\mathbf{N}_{2}=\left\{\left(\begin{array}{ll}I & b \\ 0 & I\end{array}\right) ; b=\left(\begin{array}{cc}0 & b_{2} \\ \theta b_{2} & 0\end{array}\right)\right\}$. Since the form $\Phi$ is left invariant under $\mathbf{N}_{Q}(\mathbb{A})$, and the image of $\mathbf{N}_{2}$ in $\mathbf{M}_{Q}=\mathbf{Q} / \mathbf{N}_{Q}$ is the nontrivial standard unipotent radical in $\mathbf{M}_{Q}$, it follows that the integral over $n \in N_{2} \backslash \mathbf{N}_{2}(\mathbb{A})$ of $\Phi(n h)$ is 0 . Indeed, $m \mapsto \Phi(m h)$ is a cusp form on $\mathbf{M}(\mathbb{A})_{Q}$ for every $h$ in $\mathbf{C}_{\theta}(\mathbb{A})$. Consequently there is no contribution to the summation formula from cuspidal $\rho$ on the parabolic $\mathbf{Q}(\mathbb{A})$. The one-dimensional $\rho$ on $\mathbf{Q}(\mathbb{A})$ do not contribute to the summation formula either, as noted in the previous section. 
12. Comparison. Fix a nontrivial additive character $\boldsymbol{\psi}$ of $\mathbb{A} / F$, and an element $\theta$ in $F-F^{2}$. Comparing the geometric sides of the Fourier summation formulae on $\mathbf{G}(\mathbb{A})=$ $\operatorname{GSp}(4, \mathbb{A})$ (Proposition 2) and $\mathbf{H}(\mathbb{A})=\mathrm{GL}(2, \mathbb{A})$ (Proposition 4), and using the summation formulae of Propositions 7 and 10 (comparison of geometric and spectral sides) we obtain the following.

Proposition 10.4. Fix $\theta \in F-F^{2}$. For any matching test functions $f=\otimes f_{v}$ on $\mathbf{G}(\mathbb{A})$ and $f^{\prime}=\otimes f_{v}^{\prime}$ on $\mathbf{H}(\mathbb{A})$, we have that $(7.1)+(7.2)=(8.1)$, where $\chi=\chi_{\theta}$ in (7.1), (7.2).

Proof. For such matching functions $f$ and $f^{\prime}$ we have that the discrete sum $(7.1)+(7.2)$ - (8.1) is equal to the continuous sum (10.1) - (7.3). Both sides here can be expressed in terms of the Satake transform of some spherical component $f_{v}$ of $f$. A well-known argument (see, e.g., $[\mathrm{FK}]$ ) using standard unitarity estimates, the absolute convergence of the sums and products in our summation formulae, and the Stone-Weierstrass theorem, implies that both the discrete sum and the continuous integral are equal to 0 .

Note that the parameters of the representations which occur in the continuous sums (7.3) and (10.1) match, for our matching functions, using the final Remark in section 6 .

It should be emphasized that Corollary 5.1 establishes, for each $f_{v}$ in $C_{c}^{\infty}\left(G_{v} / Z_{v}\right)$, the existence of a matching $f_{v}^{\prime}$ in $C_{c}^{\infty}\left(H_{v} / Z_{v}\right)$, and conversely, for each $f_{v}^{\prime}$ the existence of a matching $f_{v}$. The proof of Proposition 10.4 uses in a crucial way Proposition 8, which asserts that corresponding spherical functions are matching. In particular, almost all of the components $f_{v}$ of $f$ are the unit element $f_{v}^{0}$ in the Hecke algebra $\mathcal{H}_{v}$ of spherical ( $K_{v}$-biinvariant) functions in $C_{c}^{\infty}\left(G_{v} / Z_{v}\right)$, and almost all of $f_{v}^{\prime}$ are the unit element $f_{v}^{\prime 0}$ in the Hecke algebra $\mathcal{H}_{v}^{\prime}$ in $C_{c}^{\infty}\left(H_{v} / Z_{v}\right)$. These are corresponding, hence matching - by Proposition 6 - hence the assumption of Proposition 10.4, that $f$ and $f^{\prime}$ are matching, makes sense.

The proof of Proposition 10.4 in fact applies to yield a stronger result, which will be useful for applications. The result is the following.

Proposition 11. Fix $\theta \in F-F^{2}$. Fix a finite set $V$ of $F$-places, including the archimedean places and those which ramify in $E / F$. At each $v \notin V$ fix an unramified $H_{v}$-module $\rho_{v}=$ $I_{H}\left(\zeta_{1 v},-\zeta_{1 v}\right)$. Put $\pi_{v}=I\left(\zeta_{1 v}, \frac{1}{2}-\zeta_{1 v}\right.$; this is an unramified $G_{v}$-module. Then for any matching test functions $f_{v}^{\prime}$ on $H_{v} / Z_{v}$ and $f_{v}$ on $G_{v} / Z_{v}$, we have

$$
\begin{aligned}
& \sum_{\rho} \sum_{\Phi^{\prime} \in \rho^{\mathbb{K}^{\prime}}(V)} W_{\psi}\left(\rho\left(f_{V}^{\prime}\right) \Phi^{\prime}\right) L_{\Phi^{\prime}}\left(\frac{1}{2}, \rho \otimes \chi_{\theta}\right)+4 \pi \sum_{\Phi^{\prime}} E_{\psi}\left(I\left(f_{V}^{\prime}, \chi_{\theta}, \frac{1}{2}\right) \Phi^{\prime}, \chi_{\theta}, \frac{1}{2}\right) \bar{\Phi}^{\prime}(1) \\
& =\sum_{\pi} m(\pi) \sum_{\Phi \in \pi^{\mathbb{K}(V)}} W_{\psi_{\theta}}\left(\pi\left(f_{V}\right) \Phi\right) P_{\theta}(\bar{\Phi}) .
\end{aligned}
$$

Here $\mathbb{K}(V)=\prod_{v \notin V} K_{v}, \mathbb{K}^{\prime}(V)=\prod_{v \notin V} K_{v}^{\prime}$, and

$$
f_{V}=\left(\otimes_{v \in V} f_{v}\right) \otimes\left(\otimes_{v \notin V} f_{v}^{0}\right) \quad \text { and } \quad f_{V}^{\prime}=\left(\otimes_{v \in V} f_{v}^{\prime}\right) \otimes\left(\otimes_{v \notin V} f_{v}^{\prime 0}\right) .
$$

The sum over $\rho$ extends over the cuspidal representations of $\operatorname{PGL}(2, \mathbb{A})$ which have a nonzero vector fixed by $\mathbb{K}^{\prime}(V)$, such that $\rho_{v}$ is the unramified constituent of $I_{H}\left(\zeta_{1 v},-\zeta_{1 v}\right)$ 
for all $v \notin V$. The sum over $\Phi^{\prime}$ ranges over an orthonormal basis of smooth vectors in the space $\rho^{\mathbb{K}^{\prime}(V)}$ of $\mathbb{K}^{\prime}(V)$-fixed vectors in $\rho$. The second sum over $\Phi^{\prime}$ is empty unless $I_{H}\left(\chi_{\theta v}, \frac{1}{2}\right) \simeq I_{H}\left(\zeta_{1 v},-\zeta_{1 v}\right)$ for all $v \notin V$, in which case the first sum, over $\rho$, is empty. Then $\Phi^{\prime}$ ranges over $I_{H}\left(\chi_{\theta}, \frac{1}{2}\right)^{\mathbb{K}^{\prime}(V)}$.

The sum over $\pi$ extends over all discrete spectrum automorphic representations of $\mathbf{G}(\mathbb{A})$ such that $\pi_{v}$ is the unramified constituent of $I\left(\zeta_{1 v}, \frac{1}{2}-\zeta_{1 v}\right)$ for all $v \notin V$. The $\Phi$ range over an orthonormal basis of smooth vectors in the space $\pi^{\mathbb{K}(V)}$ of $\mathbb{K}(V)$-fixed vectors in $\pi$.

Proof. Again, this proceeds along well known lines, using the fact that if $f_{v}$ is spherical then $\pi_{v}\left(f_{v}\right)$ acts as multiplication by the scalar $\operatorname{tr} \pi_{v}\left(f_{v}\right)$ on the (unique up to a scalar multiple when $\pi_{v}$ is irreducible) $K_{v}$-fixed vector in $\pi_{v}$, and as 0 on the orthogonal complement of this vector. Of course the trace $\operatorname{tr} \pi_{v}\left(f_{v}\right)$ is 0 unless $\pi_{v}$ is unramified, and it is an invariant finite Laurent series in $q_{v}^{\zeta_{1}}$ and $q_{v}^{\zeta_{2}}$ when $\pi_{v}=I\left(\zeta_{1}, \zeta_{2}\right)$. Note that at least one of the two sums on the side of $\mathbf{H}(\mathbb{A})$ is empty, since no cuspidal $\mathbf{H}(\mathbb{A})$-module is equivalent at almost all places to a one-dimensional representation.

The main representation theoretic application of this identity is the following. Recall that by a cuspidal representation we mean an irreducible one.

Proposition 12. Let $\rho$ be a cuspidal representation of $\operatorname{PGL}(2, \mathbb{A})$ with $L\left(\frac{1}{2}, \rho\right)=0$ and $L\left(\frac{1}{2}, \rho \otimes \chi_{\theta}\right) \neq 0$ for some $\theta$ in $F-F^{2}$. Then there exists a cuspidal representation $\pi$ of $\operatorname{PGSp}\left(4, \mathbb{A}\right.$ ) which is $\mathbf{C}_{\theta}(\mathbb{A})$-cyclic (thus $P_{\theta}(\Phi)=\int_{\mathbf{Z}(\mathbb{A}) C_{\theta} \backslash \mathbf{C}_{\theta}(\mathbb{A})} \Phi(h) d h$ is nonzero for some $\Phi$ in $\pi)$, and $\pi_{v}$ is the unramified constituent of the induced $I\left(\rho_{v}, \frac{1}{2}\right)$ for almost all $v$. Moreover, for any quadratic character $\chi=\chi_{\theta} \neq 1$ of $F^{\times} \backslash \mathbb{A}^{\times}$there exists a $\mathbf{C}_{\theta}(\mathbb{A})$-cyclic discrete-spectrum representation $\pi$ of $\operatorname{PGSp}(4, \mathbb{A})$ with $\pi_{v}$ being the unramified constituent of $I\left(\frac{1}{2}, 0 ; \chi_{v}, \chi_{v}\right)$ for almost all $v$.

Proof. Fix a set $V$ of $F$-places containing the archimedean ones and those which ramify in $E=F(\sqrt{\theta})$, such that $\rho_{u}$ is unramified for all $u \notin V$. For any matching test functions $f_{v}^{\prime}$ on $H_{v} / Z_{v}$ and $f_{v}$ on $G_{v} / Z_{v}$, the identity of Proposition 11 holds. Namely it holds where the fixed unramified $H_{v}$-modules at all $v \notin V$ are the components $\rho_{v}$ of our $\rho$. In this case $\rho$ of our proposition parametrizes the only term in the left side of the equality of Proposition 11.

Note that $\operatorname{tr} I_{H}\left(\zeta_{1},-\zeta_{1} ; f_{v}^{\prime}\right)$ is equal to $\operatorname{tr} I\left(\zeta_{1}, \frac{1}{2}-\zeta_{1} ; f_{v}\right)$. Since $L\left(\frac{1}{2}, \rho \otimes \chi_{\theta}\right) \neq 0$ and $L\left(\zeta, \rho \otimes \chi_{\theta}\right)$ lies in the span of the $L$-functions $L_{\Phi^{\prime}}\left(\zeta, \rho \otimes \chi_{\theta}\right)$, there is some $\Phi_{1}^{\prime} \in \rho$ with $L_{\Phi_{1}^{\prime}}\left(\frac{1}{2}, \rho \otimes \chi_{\theta}\right) \neq 0$.

Since $\rho$ is cuspidal it is generic. Hence there is a vector $\Phi_{2}^{\prime} \in \rho^{\mathbb{K}^{\prime}(V)}$, such that the value $W_{\psi}\left(\Phi_{2}^{\prime}\right)$ of the Whittaker function of $\Phi_{2}^{\prime}$ at the identity is nonzero. Note that the matching Corollary 6 permits us to use an arbitrary test function $f_{V}^{\prime}$. Since the operators $\rho\left(f_{V}^{\prime}\right)$ span the endomorphisms algebra of $\rho^{\mathbb{K}^{\prime}}(V)$, we may choose $f_{V}^{\prime}$ to have the property that $\rho\left(f_{V}^{\prime}\right)$ acts as 0 on each vector in $\rho$ which is orthogonal to $\Phi_{1}^{\prime}$, but it maps $\Phi_{1}^{\prime}$ to $\Phi_{2}^{\prime}$. With this choice of $f_{V}^{\prime}$ the left side of the identity of Proposition 11 reduces to single term, which is

$$
W_{\psi}\left(\Phi_{2}^{\prime}\right) L_{\bar{\Phi}_{1}^{\prime}}\left(\frac{1}{2}, \rho \otimes \chi_{\theta}\right) .
$$


This is nonzero, as is the left side, and also the right side of the identity. Hence there exists a discrete spectrum automorphic representation $\pi$ of $\operatorname{PGSp}(4, \mathbb{A})$, with $P_{\theta}\left(\Phi_{1}\right) \neq 0$ and $W_{\psi_{\theta}}\left(\Phi_{2}\right) \neq 0$ for some $\Phi_{1}, \Phi_{2}$ in $\pi$.

This $\pi$ has the property that $\operatorname{tr} \pi_{v}\left(f_{v}\right)=\operatorname{tr} I\left(\rho_{v}, \frac{1}{2} ; f_{v}\right)$ for all $v \notin V$, namely $\pi_{v}$ is the unramified irreducible constituent of $I\left(\rho_{v}, \frac{1}{2}\right)$. This constituent is the Langlands quotient $J\left(\rho_{v}, \frac{1}{2}\right)$ of $I\left(\rho_{v}, \frac{1}{2}\right)$. Langlands' theory of Eisenstein series implies that the quotient $J\left(\rho, \frac{1}{2}\right)=\otimes J\left(\rho_{v}, \frac{1}{2}\right)$ of $I\left(\rho, \frac{1}{2}\right)$ is automorphic, and defines a residual discrete spectrum representation, precisely when $L\left(\rho, \frac{1}{2}\right) \neq 0$. Our choice of $\rho$ with $L\left(\rho, \frac{1}{2}\right)=0$ guarantees that there is no non cuspidal discrete spectrum automorphic representation whose components are $J\left(\rho_{v}, \frac{1}{2}\right)$ for almost all $v$. Hence the $\pi$ which we obtained is cuspidal, as required.

The last claim of the proposition, concerning $\pi$ whose components are almost all the Langlands quotient $J\left(\frac{1}{2}, 0 ; \chi_{v}, \chi_{v}\right)$ from the Borel subgroup, can be proven similarly, but it is of little interest; see the following remark.

13. Converse. We start with a description of some GSp(4)-packets. A comparison of the trace formula of GL(4) twisted by the outer automorphism $g \mapsto{ }^{t} g^{-1}$, with the stable trace formula of GSp(4), carried out in $[\mathrm{F} 8]$ in analogy with the theory of base change from $U(3, E / F)$ to $\mathrm{GL}(3, E)$ of [F3], provides a detailed description of the packets of automorphic and admissible representations of $\operatorname{GSp}(4)$. Here we shall briefly recall the description of the packets of interest for us, from [A3], p. 32 and [F8].

If $\rho$ is a cuspidal representation of $\operatorname{PGL}(2, \mathbb{A})$ there is a global packet of $2^{r}$ global automorphic representations of $\operatorname{PGSp}(4, \mathbb{A})$ whose components are the Langlands quotient $J\left(\rho_{v}, \frac{1}{2}\right)$ of the induced $I\left(\rho_{v}, \frac{1}{2}\right)=I_{P}\left(\rho_{v}, \frac{1}{2}\right)$ at almost all places $v$ of $F$. Here $r$ is the number of discrete series components of $\rho$. Exactly half of these are discrete spectrum automorphic representations, when $r \geq 1$. When $r=0$ the single representation is discrete spectrum, necessarily residual, precisely when $L\left(\rho, \frac{1}{2}\right) \neq 0$.

One of these $2^{r-1}$ representations $(r \geq 1)$ is residual, namely non cuspidal, precisely when $L\left(\rho, \frac{1}{2}\right) \neq 0$. It is the quotient $J\left(\rho, \frac{1}{2}\right)=\otimes_{v} J\left(\rho_{v}, \frac{1}{2}\right)$. Its space is generated by the residues of the Eisenstein series $E(h, \Phi, \rho, \zeta)$ at $\zeta=\frac{1}{2}$.

When $\rho_{v}$ is square-integrable, the induced $I\left(\rho_{v}, \frac{1}{2}\right)$ is reducible, of length two (see [Sh], Proposition 6.1, p. 287, for a proof in the case where $\rho_{v}$ is cuspidal). The constituent other than $J\left(\rho_{v}, \frac{1}{2}\right)$ is non cuspidal but square-integrable, denoted here by $\pi\left(\rho_{v}\right)^{+}$. The packet of $\pi\left(\rho_{v}\right)^{+}$will contain a second member, a cuspidal $\pi\left(\rho_{v}\right)^{-}$.

The cuspidal automorphic representations in the packet of $J\left(\rho, \frac{1}{2}\right)$ are of the form $\pi=$ $\otimes \pi_{v}$, where $\pi_{v}=\pi\left(\rho_{v}\right)^{-}$for an even number of places $v$ where $\rho_{v}$ is square-integrable and $\pi \neq J\left(\rho, \frac{1}{2}\right)$ if $L\left(\rho, \frac{1}{2}\right) \neq 0$, in which case $\pi=J\left(\rho, \frac{1}{2}\right)$ is discrete spectrum, residual but not cuspidal. Namely there are $2^{r-1}$ cuspidal representations in the packet when $L\left(\rho, \frac{1}{2}\right)=0$, and $2^{r-1}-1$ when $L\left(\rho, \frac{1}{2}\right) \neq 0$, provided $r \geq 1$, and 0 when $r=0$.

When $\rho$ is a (nontrivial) quadratic character of $F^{\times} \backslash \mathbb{A}^{\times}$, the automorphic induced representation $I_{P}\left(\rho \circ \operatorname{det}, \frac{1}{2}\right)=I_{B}\left(\frac{1}{2}, 0 ; \rho, \rho\right)$ of $\operatorname{PGSp}(4, \mathbb{A})$ has a quotient $J\left(\frac{1}{2}, 0 ; \rho, \rho\right)=$ $\otimes_{v} J\left(\frac{1}{2}, 0 ; \rho_{v}, \rho_{v}\right)$. Here $J\left(\frac{1}{2}, 0 ; \rho_{v}, \rho_{v}\right)$ is the Langlands quotient of the induced $I_{P}\left(\rho_{v} \circ\right.$ $\left.\operatorname{det}, \frac{1}{2}\right)=I_{B}\left(\frac{1}{2}, 0 ; \rho_{v}, \rho_{v}\right)$. This global quotient is irreducible and residual discrete spectrum. 
The induced $I\left(\frac{1}{2}, 0 ; \rho_{v}, \rho_{v}\right), \rho_{v} \neq 1$, is reducible of length two (by $[\mathrm{KR}]$ ), and in addition to the nontempered constituent $J\left(\frac{1}{2}, 0 ; \rho_{v}, \rho_{v}\right)$ there is a square integrable but not cuspidal constituent $\pi\left(\rho_{v}\right)^{+}$. The packet of $\pi\left(\rho_{v}\right)^{+}$contains also a cuspidal member $\pi\left(\rho_{v}\right)^{-}$.

The automorphic members in the global packet of $J\left(\frac{1}{2}, 0 ; \rho, \rho\right)$ are obtained by replacing the component $J\left(\frac{1}{2}, 0 ; \rho_{v}, \rho_{v}\right), \rho_{v} \neq 1$, by the cuspidal $\pi\left(\rho_{v}\right)^{-}$, at an even number of places. They are all cuspidal, except the residual $J\left(\frac{1}{2}, 0 ; \rho, \rho\right)$. These representations were studied by Howe and Piatetski-Shapiro [HPS] by means of the Theta lifting. These examples are similar to those found in [F3] in the case of U(3): these are cuspidal representations with a finite number of cuspidal components and all other components are nontempered.

The examples related to $J\left(\rho, \frac{1}{2}\right)$, where $\rho$ is cuspidal, and the packet contains only a finite number of elements, were studied in [PS1]. They are different from the examples of [F3] on $\mathrm{U}(3)$, but similar to packets of the two-fold covering group of $\mathrm{SL}(2, \mathbb{A})$, described by Waldspurger [Wa1].

Our Proposition 12 establishes the existence of a cuspidal element in the packet of $J\left(\rho, \frac{1}{2}\right), \rho$ cuspidal, using its properties of being cyclic $\left(P_{\theta} \neq 0\right)$ and having a nonzero Fourier coefficient $W_{\psi_{\theta}}$. To prove that this packet contains a cuspidal element we used the fact that there is no residual representation when $L\left(\rho, \frac{1}{2}\right)=0$ and $\rho$ is cuspidal. But we have not proven the existence of a cuspidal member in the packet when $\rho$ is cuspidal and $L\left(\rho, \frac{1}{2}\right) \neq 0$, nor when $\rho \neq 1$ is a quadratic character (When $\rho=1$ there are no discrete spectrum representation in the packet of $\left.J\left(\rho, \frac{1}{2}\right)\right)$. These more refined results may follow on developing a suitable analogue of Kazhdan's orthogonality relations for characters (see $[\mathrm{K}])$ for the Whittaker-Period distributions introduced in the next paragraph.

We shall also consider a converse to Proposition 12. For this purpose note that a recent theorem of Waldspurger [Wa3], extending to $(\mathrm{SO}(n), \mathrm{SO}(n-1))$ a result of Aizenbud, Gourevitch, Rallis and Schiffmann [AGRS], which in turn uses ideas of Bernstein, shows that on any irreducible admissible representation $\pi_{v}$ of $G_{v}=\operatorname{PGSp}\left(4, F_{v}\right)=\mathrm{SO}\left(5, F_{v}\right)$ there exists at most one - up to a scalar multiple $-C_{\theta, v}=\mathrm{SO}\left(4, F_{v}\right)$-invariant nonzero linear form $P_{\pi_{v}}$. Here $F_{v}$ is a local nonarchimedean field of characteristic zero. The analogue for the archimedean fields $\mathbb{R}$ and $\mathbb{C}$ was proven by Binyong Sun and Chen-Bo Zhu [SZ], and in positive characteristic the work of A. Aizenbud, N. Avni, D. Gourevitch [AAG] deals with the case of $(\mathrm{GL}(n), \mathrm{GL}(n-1))$. Our case of $(\mathrm{SO}(n), \mathrm{SO}(n-1))$ is not yet done. Our claims in Proposition 13 below uses the multiplicity one theorem in positive characteristic (to define the distribution $W P$ ), so we assume its validity.

Fix such a form. Let us also fix a linear form $W_{\pi_{v}, \psi_{\theta}}$ on such a $\pi_{v}$ which transforms under the action of $N_{v}=\left\{u=\left(\begin{array}{cc}I & X \\ 0 & I\end{array}\right)\right\}$ according to multiplication by the character $\psi_{\theta}(u)$.

If $P_{\tilde{\pi}_{v}}$ denotes the invariant form on the contragredient $\tilde{\pi}_{v}$ of $\pi_{v}$, then it lies in the dual $\tilde{\pi}_{v}^{*}$ of $\tilde{\pi}_{v}$, and for each $f_{v} \in C_{c}^{\infty}\left(G_{v} / Z_{v}\right)$ the vector $\pi_{v}\left(f_{v}\right) P_{\tilde{\pi}_{v}}$ lies in the smooth part $\tilde{\tilde{\pi}}_{v} \simeq \pi_{v}$ of $\tilde{\pi}_{v}^{*}$. Associated to $\pi_{v}$ and $\psi_{v}$ we then obtain a linear form

$$
W P_{\pi_{v}, \psi_{\theta}}: f_{v} \mapsto\left\langle W_{\pi_{v}, \psi_{\theta}}, \pi_{v}\left(f_{v}\right) P_{\tilde{\pi}_{v}}\right\rangle=\sum_{\xi} W_{\pi_{v}, \psi_{v}}\left(\pi_{v}\left(f_{v}\right) \xi\right) P_{\tilde{\pi}_{v}}\left(\xi^{\vee}\right)
$$

on $C_{c}^{\infty}\left(G_{v} / Z_{v}\right)$ with the property that $W P_{\pi_{v}, \psi_{\theta}}\left({ }^{n} f_{v}^{h}\right)=\psi_{\theta}(n) W P_{\pi_{v}, \psi_{\theta}}\left(f_{v}\right)$ for ${ }^{n} f_{v}^{h}(g)=$ 
$f_{v}\left(n^{-1} g h\right), n \in N_{v}, h \in C_{\theta, v}, g \in G_{v}$. The sum over $\xi$ on the right ranges over an orthonormal basis for $\pi_{v}$, and $\xi^{\vee}$ is the dual basis for $\tilde{\pi}_{v}$. Also, $W_{\pi_{v}, \psi_{\theta}}$ lies in the dual $\pi_{v}^{*}$ of $\pi_{v}$, hence its value at $\pi_{v}\left(f_{v}\right) P_{\tilde{\pi}_{v}}$ is well-defined.

The Whittaker-Period distributions $W P_{\pi_{v}, \psi_{\theta}}$ are analogous to Harish-Chandra's characters. They have interesting properties, but we shall use only the simple fact - see Proposition 0.1 of [F7] (the distribution $\left(W_{\psi} \bar{P}\right)_{\pi}(f)$ is obtained on taking $C_{1}=N, \zeta_{1}=$ $\psi, C_{2}=C, \zeta_{2}=1, P_{1}=W_{\psi}$ and $P_{2}=P$ there) - that $W P_{\pi_{v}}$ is independent of the choice of the basis $\xi$, and that if $\pi_{1 v}, \cdots, \pi_{k v}$ are inequivalent irreducible representations, then $W P_{\pi_{1 v}, \psi_{\theta}}, \cdots, W P_{\pi_{k v}, \psi_{\theta}}$ are linearly independent distributions on $C_{c}^{\infty}\left(G_{v} / Z_{v}\right)$.

Proposition 13. Let $F$ be a global field, and fix $\theta \in F-F^{2}$. Let $\pi$ be a discrete spectrum representation of $\operatorname{PGSp}(4, \mathbb{A})$ with $P_{\theta}\left(\Phi_{1}\right) \neq 0$ and $W_{\psi_{\theta}}\left(\Phi_{2}\right) \neq 0$ for some $\Phi_{1}, \Phi_{2}$ in $\pi$. Then either there exists a cuspidal representation $\rho$ of $\mathrm{GL}(2, \mathbb{A})$ with $L\left(\frac{1}{2}, \rho \otimes \chi_{\theta}\right) \neq 0$ and $\pi_{v} \simeq J\left(\rho_{v}, \frac{1}{2}\right)$ for almost all $v$, or $\pi_{v} \simeq J\left(\frac{1}{2}, 0 ; \chi_{\theta, v}, \chi_{\theta, v}\right)$ for almost all $v$.

Proof. Choose a sufficiently large finite set $V$ containing all the $F$-places where $\pi$ or $E$ ramify, and at each $v \in V$ fix a congruence subgroup $K_{v}^{\prime} \subset K_{v}$ such that $\pi_{v}$ contains a nonzero $K_{v}^{\prime}$-fixed vector. When $F$ is a function field we shall work only with $f_{v}$ in $C_{c}^{\infty}\left(K_{v}^{\prime} \backslash G_{v} / Z_{v} K_{v}^{\prime}\right), v \in V$. The right side of the identity of Proposition 11 can be written in the form

$$
\sum_{\pi} m(\pi) \prod_{v \in V} W P_{\pi_{v}, \psi_{\theta}}\left(f_{v}\right)
$$

The sum is finite, since we fixed the ramification at all places, and $F$ is a function field. The linear independence of the forms $W P_{\pi_{v}, \psi_{\theta}}$ implies that there are $f_{v} \in C_{c}^{\infty}\left(G_{v} / / Z_{v} K_{v}\right)(v \in$ $V)$ for which the sum over $\pi$ reduces to a single nonzero contribution, that which is parametrized by $\pi$. Here we used Corollary 6 , which permits us to use any test functions $f_{v}$. In particular, the right side, associated with $G$, is nonzero. Hence so is the left side, establishing the existence of $\rho$, or $J\left(\frac{1}{2}, 0 ; \chi_{\theta}, \chi_{\theta}\right)$, associated with $\pi$, as asserted.

When $F$ is a number field, we can use generalized linear independence of characters, using the fact that spherical functions are matching. The displayed expression above ranges only over the $\pi$ with an a-priori fixed unramified component at each place outside $V$. The sum is finite by the rigidity theorem of [F8], which follows from the comparison of [F8] of trace formulae on $\mathrm{GSp}(4)$ and $\mathrm{GL}(4)$. We continue as in the first part of this proof.

14. Split cycles. We have studied above cusp forms on $\mathbf{G}(\mathbb{A})=\operatorname{PGSp}(4, \mathbb{A})$ cyclic with respect to the subgroup $\mathbf{C}_{\theta}(\mathbb{A}), \theta \in F-F^{2}$, defined in section 2. There is no analogous theory with $\mathbf{C}(\mathbb{A})=\mathbf{C}_{0}(\mathbb{A})$ replacing $\mathbf{C}_{\theta}(\mathbb{A})$, since there are no cusp forms on $\mathbf{G}(\mathbb{A})$ with nonzero $\mathbf{C}(\mathbb{A})$-cycles. This is the content of [PS1], Corollary 6.2 , proven by means of the Theta correspondence. We shall outline next a spectral proof, using a Fourier summation formula, in the spirit of this paper, of that fact. As usual, fix a character $\boldsymbol{\psi} \neq 1$ of $\mathbb{A} / F$ into the multiplicative groups of the complex numbers, and introduce a character $\psi_{B}$ of $\mathbf{N}_{B}(\mathbb{A})=\left\{n=\left(\begin{array}{llll}1 & x & * & * \\ 0 & 1 & y & * \\ 0 & 0 & 1 & -x \\ 0 & 0 & 0 & 1\end{array}\right)\right\}$ by $\psi_{B}(n)=\boldsymbol{\psi}(x+y)$. The Whittaker function of a cusp form $\Phi$ on $\mathbf{G}(\mathbb{A})$ is defined by $W_{\Phi}(g)=\int_{N_{B} \backslash \mathbf{N}_{B}(\mathbb{A})} \Phi(n g) \bar{\psi}(n) d n$. A cuspidal $\mathbf{G}(\mathbb{A})$-module 
$\pi$ is called generic if $W_{\Phi} \neq 0$ for some $\Phi$ in $\pi$. Put $z_{\Phi}(g)=\int_{Z_{Q} \backslash \mathbf{Z}(\mathbb{A})_{Q}} \Phi(z g) d z$, where - as in section $2-\mathbf{Z}_{Q}$ is the center of the unipotent radical $\mathbf{N}_{Q}$ of the parabolic subgroup $\mathbf{Q}$ of $\mathbf{G}=\mathrm{GSp}(4)$. Denote by $\mathbf{R}$ the standard maximal unipotent subgroup of the standard Levi subgroup $\mathbf{M}_{Q}$ of $\mathbf{Q}$. As in [PS1], Lemma 6.2, we have the following.

Proposition 14. For any cusp form $\Phi$ on $\mathbf{G}(\mathbb{A})$, we have that $z_{\Phi}(g)=\sum_{\gamma \in R \backslash M_{Q}} W_{\Phi}(\gamma g)$.

Proof. The quotient $\mathbf{Z} \backslash \mathbf{Q} / \mathbf{Z}_{Q}$ is naturally isomorphic to the subgroup $\mathbf{D}=\left\{\left(\begin{array}{l}* * * \\ * * * \\ 0\end{array}\right.\right.$ of $\mathrm{GL}(3)$, and $z_{\Phi}(\delta g)=z_{\Phi}(g)$ for all $\delta \in D$. Since $\Phi$ is cuspidal, $\int_{Y \backslash \mathbb{Y}} z_{\Phi}(y d) d y=0$ for all $d \in \mathbf{D}(\mathbb{A})$, where $\mathbf{Y}$ is any of the unipotent subgroups $\mathbf{Y}_{1}=\left\{\left(\begin{array}{lll}1 & 0 & * \\ 0 & 1 & * \\ 0 & 0 & 1\end{array}\right)\right\}$ of $\mathbf{Y}_{2}=$ $\left\{\left(\begin{array}{lll}1 & * & * \\ 0 & 1 & 0 \\ 0 & 0 & 1\end{array}\right)\right\}$ of $\mathbf{D}$. The stabilizer of the character $\psi_{1}(y)=\boldsymbol{\psi}\left(y_{2}\right), y=\left(\begin{array}{lll}1 & 0 & y_{1} \\ 0 & 1 & y_{2} \\ 0 & 0 & 1\end{array}\right)$, of $\mathbb{Y}_{1}$ is $\mathbf{D}(\mathbb{A})_{1}, \mathbf{D}_{1}=\left\{\left(\begin{array}{ccc}* & * & * \\ 0 & 1 & * \\ 0 & 0 & 1\end{array}\right)\right\}$. Hence the Fourier expansion of $z_{\Phi}$ along $\mathbb{Y}_{1}$ is

$$
z_{\Phi}(d)=\sum_{\delta \in D_{1} \backslash D} z_{\Phi, 1}(\delta d), \quad z_{\Phi, 1}(d)=\int_{Y_{1} \backslash \mathbb{Y}_{1}} z_{\Phi}(y d) \bar{\psi}_{1}(y) d y .
$$

Taking the Fourier expansion of $z_{\Phi, 1}$ along the subgroup $\mathbf{Y}_{1}=\left\{\left(\begin{array}{lll}1 & * & 0 \\ 0 & 1 & 0 \\ 0 & 0 & 1\end{array}\right)\right\}$, and noting that the constant term is zero (since $\Phi$ is cuspidal), we obtain $z_{\Phi}(d)=\sum_{\delta \in N \backslash D} W_{\Phi}(\delta d)$, where $N$ is the unipotent upper triangular subgroup of $D \simeq Q / Z_{Q}$, as required.

This Proposition implies that, if $\Phi$ is a cusp form on $\mathbf{G}(\mathbb{A})$ with $\int_{\mathbf{Z}(\mathbb{A}) C \backslash \mathbf{C}(\mathbb{A})} \Phi(h) d h \neq 0$, namely its restriction to $\mathbf{C}(\mathbb{A})$ is not orthogonal to the constant functions and hence it is not cuspidal (on $\mathbf{C}(\mathbb{A})$ ), then $z_{\Phi} \neq 0$ (otherwise the restriction of $\Phi$ to $\mathbf{C}(\mathbb{A}$ ) would be cuspidal), and $W_{\Phi} \neq 0$ namely $\Phi$ lies in a generic $\pi$. We are led then to consider the sum in the following.

Proposition 15. Let $f$ be a cuspidal test function on $\mathbf{G}(\mathbb{A})$ (e.g., it has a cuspidal component). Let $\{\Phi\}$ denote an orthonormal basis of the space of cusp forms on $\mathbf{G}(\mathbb{A})$. Then

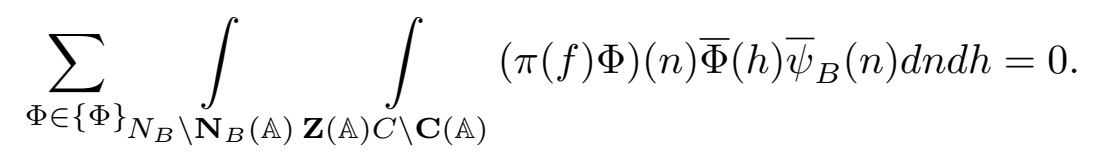

Proof. The sum of the proposition is the spectral side of a Fourier summation formula. It suffices to compute its geometric side,

$$
\int_{\mathbf{N}(\mathbb{A})_{B} / N_{B} \mathbf{Z}(\mathbb{A}) C \backslash \mathbf{C}(\mathbb{A})} \sum_{\gamma \in Z \backslash G} f(n \gamma h) \bar{\psi}_{B}(n) d n d h .
$$


Proposition 1(b) asserts that $G=N C \cup N A \gamma_{1} C \cup N \gamma_{2} C \cup N A \gamma_{3} C$. To show that our sum vanishes it suffices to note that $\psi_{B}$ is nontrivial on $\gamma_{i} \mathbf{C}(\mathbb{A}) \gamma_{i}^{-1} \cap \mathbf{N}(\mathbb{A})_{B},(i=1,2,3,4), \gamma_{4}=$

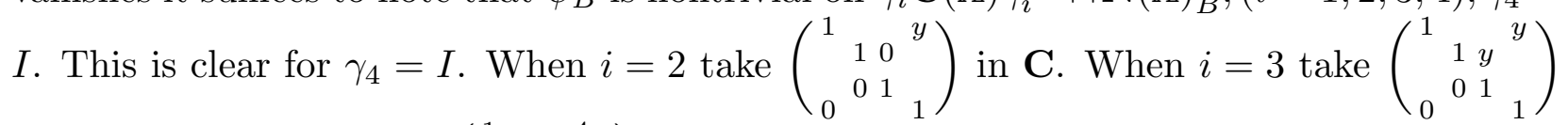
(in $\mathbf{C}$ ). When $i=1$ take $\left(\begin{array}{lll}1 & 1 & 4 x \\ & 1 & 4 \\ 0 & 1 & 1\end{array}\right)$ (in $\mathbf{C}$ ). The proposition follows.

To show that there are no cusp forms on $\mathbf{G}(\mathbb{A})$ with nonzero $\mathbf{C}(\mathbb{A})$-cycles it remains to (extend Proposition 15 to a general test function $f$ and) isolate any single cusp form $\Phi$ which occurs in the sum. When $F$ is a function field, this separation is performed in a similar context in the Appendix below.

15. Appendix. Invariance of Fourier coefficients of cyclic automorphic forms.

Let $\mathbf{G}$ be a reductive group scheme over a global field $F$, and $\mathbf{P}=\mathbf{M N}$ a parabolic $F$-subgroup with Levi subgroup $\mathbf{M}$ and unipotent radical $\mathbf{N}$. Put $G=\mathbf{G}(F), P=\mathbf{P}(F)$, $N=\mathbf{N}(F), \ldots$ for the groups of $F$-rational points, and $\mathbf{G}(\mathbb{A}), \mathbf{P}(\mathbb{A}), \mathbf{N}(\mathbb{A}), \ldots$ for the group of points over the ring $\mathbb{A}$ of $F$-adèles. Let $\psi: N \backslash \mathbf{N}(\mathbb{A}) \rightarrow \mathbb{C}^{\times}$be a character, and let $\phi$ be an automorphic form on $\mathbf{G}(\mathbb{A})$ which transforms trivially under $\mathbf{Z}(\mathbb{A})$, where $\mathbf{Z}$ is the center of $\mathbf{G}$ (thus $\phi \in L^{2}(G \mathbf{Z}(\mathbb{A}) \backslash \mathbf{G}(\mathbb{A}))$ ). The $(\psi$-) Fourier coefficient of $\phi$ (along the compact homogeneous space $N \backslash \mathbf{N}(\mathbb{A}))$ is defined to be $W_{\psi}(\phi)=\int_{N \backslash \mathbf{N}(\mathbb{A})} \phi(n) \bar{\psi}(n) d n$. The Levi subgroup $\mathbf{M}(\mathbb{A})$ acts on $\mathbf{N}(\mathbb{A})$ by conjugation. The stabilizer of $\psi$ is

$$
\operatorname{Stab}_{\mathbf{M}(\mathbb{A})}(\psi)=\left\{m \in \mathbf{M}(\mathbb{A}) ; \psi\left(m n m^{-1}\right)=\psi(n) \quad \text { all } n \in \mathbf{N}(\mathbb{A})\right\} .
$$

It is a subgroup of $\mathbf{M}(\mathbb{A})$, and its subgroup of rational points is denoted by $\operatorname{Stab}_{M}(\psi)$. The "generalized Whittaker" functional $W_{\psi}(\phi)$ is clearly $\operatorname{Stab}_{M}(\psi)$-invariant, $W_{\psi}(r(m) \phi)=$ $W_{\psi}(\phi)$, where $(r(g) \phi)(h)=\phi(h g)$, since $\phi$ is an automorphic form. In general $W_{\psi}(\phi)$ is not $\operatorname{Stab}_{\mathbf{M}(\mathbb{A})}(\psi)$-invariant. The purpose of this Appendix is to show that under some natural geometric conditions (on the group), cyclic cusp forms do have the property that $W_{\psi}$ is invariant under the group of adèle points of the connected component of the identity $\operatorname{Stab}_{\mathbf{M}}^{\circ}(\psi)$ in $\operatorname{Stab}_{\mathbf{M}}(\psi)$.

Denote by $L_{0}(G \mathbf{Z}(\mathbb{A}) \backslash \mathbf{G}(\mathbb{A}))$ the space of cusp forms, namely $\phi$ in $L^{2}(G \mathbf{Z}(\mathbb{A}) \backslash \mathbf{G}(\mathbb{A}))$ such that $\int_{N^{\prime} \backslash \mathbf{N}(\mathbb{A})^{\prime}} \phi\left(n^{\prime} g\right) d n^{\prime}=0$ for all $g \in \mathbf{G}(\mathbb{A})$ and any proper $F$-parabolic subgroup $\mathbf{P}^{\prime}=\mathbf{M}^{\prime} \mathbf{N}^{\prime}$ of $\mathbf{G}$. A cuspidal $\mathbf{G}(\mathbb{A})$-module is an irreducible constituent of the representation of $\mathbf{G}(\mathbb{A})$ by right translation on $L_{0}=L_{0}(G \mathbf{Z}(\mathbb{A}) \backslash \mathbf{G}(\mathbb{A}))$. Cusp forms are rapidly decreasing on a Siegel domain. Let $\mathbf{C}$ be an $F$-subgroup of $\mathbf{G}$. Assuming that the cycle $\mathbf{Z}(\mathbb{A}) C \backslash \mathbf{C}(\mathbb{A})$ is of finite volume, the period integral $P_{C}(\phi)=\int_{\mathbf{Z}(\mathbb{A}) C \backslash \mathbf{C}(\mathbb{A})} \phi(h) d h$ converges. A cuspidal representation $\pi \subset L_{0}$ is called cyclic if it contains a form $\phi$ with a nonzero period $P_{C}(\phi)$ over the cycle $\mathbf{Z}(\mathbb{A}) C \backslash \mathbf{C}(\mathbb{A})$. Any form $\phi$ in such $\pi$ has a nonzero period $P_{C^{\prime}}(\phi) \neq 0$, where $\mathbf{C}^{\prime}(\mathbb{A})$ is conjugate to $\mathbf{C}(\mathbb{A})$ over $\mathbf{G}(\mathbb{A})$.

Theorem A. Denote by $\{\delta\}$ a set of representatives in $G$ for the double coset space $N \backslash G / C$, and by $\{\delta\}^{\prime}$ its subset of $\delta$ such that $\psi$ is 1 on $\mathbf{N}(\mathbb{A}) \cap \delta \mathbf{C}(\mathbb{A}) \delta^{-1}$. Suppose that $\operatorname{Stab}_{\mathbf{M}(\mathbb{A})}^{\circ}(\psi)$ is contained in $\delta \mathbf{C}(\mathbb{A}) \delta^{-1}$ for all $\delta \in\{\delta\}^{\prime}$. If $\pi$ is a cyclic cuspidal $\mathbf{G}(\mathbb{A})$ module with a cuspidal component, then $W_{\psi}(\phi)$ is $\operatorname{Stab}_{\mathbf{M}(\mathbb{A})}^{\circ}(\psi)$-invariant for all $\phi$ in $\pi$. 
Remarks. When $\mathbf{G}=\mathrm{GL}(k), \mathbf{M}=$ the diagonal subgroup, $\mathbf{N}=$ the unipotent upper triangular subgroup, and $\psi(n)=\boldsymbol{\psi}\left(\sum_{1 \leq i<k} n_{i, i+1}\right)$, where $\boldsymbol{\psi}: \mathbb{A} / F \rightarrow \mathbb{C}^{\times}$is a nontrivial additive character, and $n=\left(n_{i j}\right)$, then the stabilizer of $\psi$ in $\mathbf{M}(\mathbb{A})$ is $\mathbf{Z}(\mathbb{A})$, and no new information is provided by the Theorem on the generic Fourier coefficient $W_{\psi}(\phi)$.

The Theorem applies (nontrivially) when the character $\psi$ is a degenerate character, when viewed as a character of the unipotent radical of the minimal parabolic subgroup. We shall discuss below an example where the Theorem applies nontrivially. This example, concerning GSp(4), has also been treated by Piatetski-Shapiro [PS2] by means of the theta-lifting. Our approach relies on an application of a Fourier summation formula. Although much more recent, this approach has the advantage of being conceptually simpler. A disadvantage of this technique is that it deals with the entire automorphic spectrum. The presence of contributions from the continuous spectrum, expressed in terms of Eisenstein series, leads to technical difficulties. To avoid encountering these in this Appendix, we work with cuspidal representations with a cuspidal component. These technical difficulties can be handled as in [F1], where $\mathbf{G}=\operatorname{PGL}(k), \mathbf{C}=\mathrm{GL}(k-1)$, and $\psi$ is the degenerate character $\psi(n)=\boldsymbol{\psi}\left(n_{1,2}+n_{2, k}\right)$ of the unipotent upper triangular subgroup. Yet in the case of [F1] there are no cuspidal $\mathbf{G}(\mathbb{A})$-modules cyclic over $C \backslash \mathbf{C}(\mathbb{A})$, while in the PGSp(4) example discussed below there are such cuspidal representations, and we content ourselves here with the form the Theorem takes, without launching into deep analysis. Another simplifying assumption which we make is to take $F$ to be a function field, to avoid dealing with the archimedean places.

Proof. This is based on an application of the Fourier summation formula for a test function $f=\otimes f_{v}$, product over all places $v$ of the global field $F$, where $f_{v} \in C_{c}^{\infty}\left(Z_{v} \backslash G_{v}\right)$ for all $v$, and $f_{v}$ is the unit element $f_{v}^{0}$ of the convolution algebra of spherical, namely $K_{v}$-biinvariant, compactly supported functions on $Z_{v} \backslash G_{v}$, for almost all $v$. Our notations are standard: $F_{v}$ is the completion of $F$ at the place $v$, we put $G_{v}=\mathbf{G}\left(F_{v}\right), Z_{v}=\mathbf{Z}\left(F_{v}\right), \ldots$, and $K_{v}$ is a hyperspecial maximal compact open subgroup of $G_{v}$. Implicit is a choice of a Haar measure $d g_{v}$ on $G_{v} / Z_{v}$ such that the product $\prod_{v}\left|K_{v}\right|,\left|K_{v}\right|=\operatorname{vol}\left(K_{v}\right)$, converges, thus of a global Haar measure $d g=\otimes d g_{v}$. The subscript " $c$ " means compactly supported, and the superscript " $\infty$ " indicates smooth, namely locally constant in the nonarchimedean case.

The convolution operator $(r(f) \phi)(g)=\int_{\mathbf{Z}(\mathbb{A}) \backslash \mathbb{G}} f(h) \phi(g h) d h$ on $L^{2}(G \mathbf{Z}(\mathbb{A}) \backslash \mathbf{G}(\mathbb{A}))$ is easily seen to be an integral operator $(r(f) \phi)(g)=\int_{\mathbf{Z}(\mathbb{A}) G \backslash \mathbf{G}(\mathbb{A})} K_{f}(g, h) \phi(h) d h$ with kernel $K_{f}(g, h)=\sum_{\gamma \in Z \backslash G} f\left(g^{-1} \gamma h\right)$. On the other hand, if some component - say $f_{v_{1}}$ - of $f$, is a supercusp form (we shall assume this from now on), then the operator $r(f)$ factorizes through the natural projection from $L^{2}\left(G \mathbf{Z}(\mathbb{A}) \backslash \mathbf{G}(\mathbb{A})\right.$ ) onto $L_{0}(G \mathbf{Z}(\mathbb{A}) \backslash \mathbf{G}(\mathbb{A})$ ) (see $[\mathrm{F} 4]$ ). The restriction $r_{0}(f)$ of $r(f)$ to $L_{0}$ has the kernel $K_{f}^{0}(g, h)=\sum_{\pi} \sum_{\phi}(\pi(f) \phi)(g) \bar{\phi}(h)$. Here $\pi$ ranges over a set of representatives for the equivalence classes of the irreducible constituents of $L_{0}$, while $\phi$ ranges over an orthonormal basis $\{\phi\}$ of smooth vectors in the $\pi$-isotypic component of $L_{0}$. It is well-known that the multiplicity of $\pi$ in $L_{0}$ is finite.

Now for our $f$, which has a cuspidal component, we have $K_{f}(n, h)=K_{f}^{0}(n, h)$. We multiply both sides by $\bar{\psi}(n)$ and integrate over $n$ in $N \backslash \mathbf{N}(\mathbb{A})$ and $h$ in $C \backslash \mathbf{C}(\mathbb{A})$, to obtain 
the Fourier summation formula. It is

$$
\begin{aligned}
& \sum_{\pi} \sum_{\phi} W_{\psi}(\pi(f) \phi) P(\bar{\phi})=\int_{\mathbf{N}(\mathbb{A}) / N} \int_{C \backslash \mathbf{C}(\mathbb{A})} \sum_{\gamma \in Z \backslash G} f(n \gamma h) \bar{\psi}(n) d n d h \\
& =\int_{\mathbf{N}(\mathbb{A}) / N} \int_{C \backslash \mathbf{C}(\mathbb{A})} \sum_{\delta} \sum_{\zeta \in C} \sum_{\nu \in N / N \cap \delta C \delta^{-1}} f(n \nu \delta \zeta h) \bar{\psi}(n) d n d h,
\end{aligned}
$$

where $\delta$ ranges over a set of representatives in $G$ for the double coset space $N \backslash G / C$, thus $G=\bigcup_{\delta} N \delta C$ (disjoint union). This is

$$
\begin{aligned}
& =\sum_{\delta} \int_{\mathbf{C}(\mathbb{A}) \mathbf{N}(\mathbb{A}) / N \cap \delta C \delta^{-1}} f(n \delta h) \bar{\psi}(n) d n d h \\
& =\sum_{\delta}^{\prime}\left|\mathbf{N}(\mathbb{A}) \cap \delta \mathbf{C}(\mathbb{A}) \delta^{-1} / N \cap \delta C \delta^{-1}\right| \int_{\mathbf{C}(\mathbb{A}) \mathbf{N}(\mathbb{A}) / \mathbf{N}(\mathbb{A}) \cap \delta \mathbf{C}(\mathbb{A}) \delta^{-1}} f(n \delta h) \bar{\psi}(n) d n d h .
\end{aligned}
$$

The last sum ranges over the subset of the $\delta$ for which $\psi$ is 1 on $\mathbf{N}(\mathbb{A}) \cap \delta \mathbf{C}(\mathbb{A}) \delta^{-1}$. The last expression is named the "geometric side" of the Fourier summation formula, while the initial expression is the "spectral side", for our test function $f$.

We shall compare the summation formula for $f$ with that for ${ }^{s} f(g)=f\left(s^{-1} g\right)$, for any $s$ in $\operatorname{Stab}_{\mathbf{M}(\mathbb{A})}^{0}(\psi)$. Note that

$$
\begin{aligned}
& \pi\left({ }^{s} f\right) \phi(u)=\int{ }^{s} f(g) \phi(u g)=\int f\left(s^{-1} g\right) \phi(u g) \\
& =\int f(g) \phi(u s g)=(\pi(f) \phi)(u s)=\pi(s)(\pi(f) \phi)(u) .
\end{aligned}
$$

If $\left\{\phi_{\alpha}\right\}$ denotes an orthonormal basis of the $\pi$-isotypic component of $L_{0}$, then $\phi=$ $\sum_{\alpha}\left(\phi, \phi_{\alpha}\right) \phi_{\alpha}$, where $\left(\phi, \phi^{\prime}\right)=\int_{\mathbf{Z}(\mathbb{A}) G \backslash \mathbf{G}(\mathbb{A})} \phi(g) \bar{\phi}^{\prime}(g) d g$ is a nondegenerate sesqui-linear form on $L_{0}$. Then

$$
\begin{aligned}
& \pi\left({ }^{s} f\right) \phi=\pi(s) \pi(f) \phi=\sum_{\alpha}\left(\pi(s) \pi(f) \phi, \phi_{\alpha}\right) \phi_{\alpha} \\
& =\sum_{\alpha}\left(\pi(f) \phi, \pi\left(s^{-1}\right) \phi_{\alpha}\right) \phi_{\alpha}=\sum_{\alpha}\left(\phi, \pi\left(f^{*}\right) \pi\left(s^{-1}\right) \phi_{\alpha}\right) \phi_{\alpha},
\end{aligned}
$$

where $f^{*}(g)=f\left(g^{-1}\right)$. Consequently

$$
\begin{aligned}
& \sum_{\phi} W_{\psi}\left(\pi\left({ }^{s} f\right) \phi\right) P(\bar{\phi})=\sum_{\phi} \sum_{\alpha} W_{\psi}\left(\phi_{\alpha}\right)\left(\phi, \pi\left(f^{*}\right) \pi\left(s^{-1}\right) \phi_{\alpha}\right) P(\bar{\phi}) \\
& =\sum_{\alpha} W_{\psi}\left(\phi_{\alpha}\right) P\left(\overline{\sum_{\phi}\left(\pi\left(f^{*}\right) \pi\left(s^{-1}\right) \phi_{\alpha}, \phi\right) \phi}\right) \\
& =\sum_{\alpha} W_{\psi}\left(\phi_{\alpha}\right) P\left(\overline{\pi\left(f^{*}\right) \pi\left(s^{-1}\right) \phi_{\alpha}}\right)=\sum_{\phi} W_{\psi}(\pi(s) \phi) P\left(\overline{\pi\left(f^{*}\right) \phi}\right),
\end{aligned}
$$


where to obtain the last expression we choose the orthonormal basis $\left\{\phi_{\alpha}\right\}$ to be $\{\pi(s) \phi\}$.

In summary,

$$
\begin{aligned}
& \sum_{\pi} \sum_{\phi} W_{\psi}(\pi(s) \phi) P\left(\overline{\pi\left(f^{*}\right) \phi}\right)=\sum_{\pi} \sum_{\phi} W_{\psi}\left(\pi\left(^{s} f\right) \phi\right) P(\bar{\phi}) \\
& =\sum_{\delta}^{\prime} \operatorname{vol}(\delta) \int_{\mathbf{C}(\mathbb{A}) \mathbf{N}(\mathbb{A}) / \mathbf{N}(\mathbb{A}) \cap \delta C \delta^{-1}} f\left(s^{-1} n \delta h\right) \bar{\psi}(n) d n d h,
\end{aligned}
$$

where $\operatorname{vol}(\delta)=\left|\mathbf{N}(\mathbb{A}) \cap \delta \mathbf{C}(\mathbb{A}) \delta^{-1} / N \cap \delta C \delta^{-1}\right|$, by the Fourier summation formula for ${ }^{s} f$,

$$
=\sum_{\delta}^{\prime} \operatorname{vol}(\delta) \cdot \int_{\mathbf{C}(\mathbb{A}) \mathbf{N}(\mathbb{A}) / \mathbf{N}(\mathbb{A}) \cap \delta \mathbf{C}(\mathbb{A}) \delta^{-1}} f\left(n s^{-1} \delta h\right) \psi(n) d n d h,
$$

since $s \in \operatorname{Stab}_{\mathbf{M}(\mathbb{A})}(\psi)$,

$$
=\sum_{\delta}^{\prime} \operatorname{vol}(\delta) \cdot \int_{\mathbf{C}(\mathbb{A}) \mathbf{N}(\mathbb{A}) / \mathbf{N}(\mathbb{A}) \cap \delta \mathbf{C}(\mathbb{A}) \delta^{-1}} f(n \delta h) \psi(n) d n d h,
$$

since $s \in \delta \mathbf{C}(\mathbb{A}) \delta^{-1}$ by the assumption of the theorem,

$$
=\sum_{\pi} \sum_{\phi} W_{\psi}(\phi) P\left(\overline{\pi\left(f^{*}\right) \phi}\right)
$$

by the Fourier summation formula for $f$ (and the line following the definition of $f^{*}$ ).

The theorem concerns a form $\phi_{1}$ in a fixed cuspidal $\mathbf{G}(\mathbb{A})$-module $\pi_{1}$, whose component at the finite place $v_{1}$ is cuspidal. There is a finite set $V$ of $F$-places, containing the archimedean places and $v_{1}$, such that not only $\pi_{1 v}$ is unramified for all $v$ outside $V$, but also $\phi_{1}$ is $K_{v^{-}}$ invariant for all such $v$. The part $f^{V}=\otimes_{v \notin V} f_{v}$ of $f$ outside $V$ can be chosen such that $f_{v}$ is the unit element $f_{v}^{0}$ for almost all $v$, and $f_{v}$ is any $K_{v}$-spherical function at the remaining finite set of places. For any cusp form $\phi$, say in the cuspidal $\mathbf{G}(\mathbb{A})$-module $\pi$, the operator $\pi_{v}\left(f_{v}\right)$ acts on $\phi$ by multiplication by the character $\operatorname{tr} \pi_{v}\left(f_{v}\right)$ if $\phi$ is $K_{v}$-invariant and as 0 otherwise. A standard argument of "generalized linear independence of characters" (see, e.g., $[\mathrm{FK}]$ ), based on the absolute convergence of the spectral side of the Fourier summation formula, standard unitarity estimates on the Hecke eigenvalues of the unramified $\pi_{v}$, and the Stone-Weierstrass theorem, permit deducing the following identity for all $f_{V}=\otimes_{v \in V} f_{v}$ such that $f_{v_{1}}$ is a cuspidal function.

$$
\sum_{\pi} \sum_{\phi} W_{\psi}(\pi(s) \phi) P(\overline{\pi(f) \phi})=\sum_{\pi} \sum_{\phi} W_{\psi}(\phi) P(\overline{\pi(f) \phi})
$$

where $\pi$ ranges over the cuspidal $\mathbf{G}(\mathbb{A})$-modules with $\pi_{v} \simeq \pi_{1 v}$ for all $v \notin V$, and $\phi$ ranges over an orthonormal basis for the space $\pi^{\mathbb{K}(V)}$ of $\mathbb{K}(V)=\prod_{v \notin V} K_{v}$-invariant vectors in the $\pi$-isotypic part of $L_{0}$. 
We shall now view the cuspidal representation $\pi$ as an abstract representation of $\mathbf{G}(\mathbb{A})$, and fix an isomorphism of $\pi$ with $\otimes \pi_{v}$. Any $\mathbb{K}(V)$-fixed smooth form $\phi$ in the space of the cuspidal $\pi=\otimes \pi_{v}$ corresponds to a linear combination of finitely many vectors $\xi^{V} \otimes\left(\otimes_{v \in V} \xi_{i v}\right), 1 \leq i \leq k$, where $\xi^{V}$ is the (unique up to scalar multiple) $\mathbb{K}(V)$-fixed nonzero vector in $\pi^{V}=\otimes_{v \notin V} \pi_{v}$, and $\xi_{i v} \in \pi_{v}$. We claim that each of these products corresponds to a cusp form. Since $\pi_{v}$ is admissible there is some sufficiently small good ([B]) compact open subgroup (e.g. a congruence subgroup) $K_{1 v}$ of $G_{v}$ such that $\xi_{i v}(1 \leq$ $i \leq k)$ are $\pi_{v}\left(K_{1 v}\right)$-invariant. The algebra $\left\{\pi_{v}\left(f_{v}\right) ; f_{v} \in C_{c}\left(K_{1 v} \backslash G_{v} / K_{1 v}\right)\right\}$ is the algebra of endomorphisms of the finite dimensional (since $\pi_{v}$ is admissible) module $\pi_{v}^{K_{v}}$. In particular there exists a $K_{1 v}$-biinvariant $f_{v}$ such that $\pi_{v}\left(f_{v}\right)$ acts as an orthogonal projection on the space generated by $\xi_{1 v}$. For a suitable choice of such $f_{v}(v \in V)$ we have that $\pi_{V}\left(f_{V}\right) \phi$ corresponds to a factorizable vector $\xi^{V} \otimes\left(\otimes_{v \in V} \xi_{1 v}\right)$. Consequently, in order to prove that $W_{\psi}\left(\pi(s) \phi_{1}\right)=W_{\psi}\left(\phi_{1}\right)$, we may assume that the cusp form $\phi_{1}$ corresponds to a factorizable vector. Moreover, multiplying by a scalar we may assume that the orthonormal basis $\{\phi\}$ of $\pi_{1}$ is chosen to include our form $\phi_{1}$, which corresponds to a factorizable vector.

We are assuming that $\pi_{1}$ is cyclic, namely that there exists a form $\phi_{2}$, which can be taken to be in the orthonormal basis $\{\phi\}$ of $\pi_{1}$, such that $P\left(\phi_{2}\right) \neq 0$. Thus $\phi_{2}=\phi_{1}$ or $\left(\phi_{1}, \phi_{2}\right)=0$. The argument of the previous paragraph implies that $\phi_{2}$ can also be assumed to correspond to a factorizable vector. Thus $\phi_{2}$ corresponds to $\xi^{V} \otimes\left(\otimes_{v \in V} \xi_{2 v}\right)$. At the place $v_{1}$ we take $f_{v_{1}}(g)=d\left(\pi_{1 v_{1}}\right)\left(\pi_{1 v_{1}}(g) \xi_{1 v}, \xi_{2 v}\right)$. The complex number $d\left(\pi_{1 v_{1}}\right)$ is the formal degree of $\pi_{1 v_{1}}$. This is a matrix coefficient of the cuspidal $G_{v}$-module $\pi_{1 v_{1}}$. It lies in $C_{c}^{\infty}\left(Z_{v_{1}} \backslash G_{v_{1}}\right)$. By the Schur orthonormality relations it has the property that $\pi_{v_{1}}\left(f_{v_{1}}\right)$ acts as 0 unless $\pi_{v_{1}}=\pi_{1 v_{1}}$, and then $\pi_{1 v_{1}}\left(f_{v_{1}}\right)$ acts as 0 on any vector perpendicular to $\xi_{1 v_{1}}$, while $\pi_{1 v_{1}}\left(f_{v_{1}}\right) \xi_{1 v_{1}}=\xi_{2 v_{1}}$. We conclude that the identity (1) holds where $\pi$ ranges over the cuspidal $\mathbf{G}(\mathbb{A})$-modules with $\pi_{v} \simeq \pi_{1 v}$ for all $v \notin V$ and for $v=v_{1}$, and $\phi$ ranges over an orthonormal basis of the space $\pi^{\mathbb{K}(V)}$ of the form $\xi^{V} \otimes \xi_{1 v_{1}} \otimes \xi_{V_{1}}$, where $V_{1}=V-\left\{v_{1}\right\}$ and $\xi_{V_{1}} \in \pi_{V_{1}}=\otimes_{v \in V_{1}} \pi_{v}$.

Note that the distribution $f \mapsto \sum_{\phi} W_{\psi}(\phi) P(\overline{\pi(f) \phi})$, where $\phi$ ranges over an orthonormal basis consisting of smooth vectors in the space of the irreducible cuspidal $\pi$, is independent of the choice of the basis $\{\phi\}$. Consequently, in (1), the double sums can be expressed as the product by the multiplicity of $\pi_{1}$ in $L_{0}(\mathbf{Z}(\mathbb{A}) G \backslash \mathbf{G}(\mathbb{A})$ ) (same finite number on both sides), of a sum over an orthonormal basis $\{\phi\}$ of the $\mathbb{K}\left(V_{1}\right)$-fixed smooth vectors in $\pi_{1}$. Moreover, we may assume that the basis $\{\phi\}$ consists of factorizable vectors. In fact, fix a compact open subgroup $K_{1 v}\left(v \in V_{1}\right)$ such that $\phi_{1}, \phi_{2}$ are $\pi_{1 v}\left(K_{1 v}\right)$-invariant for all $v \in V_{1}$. For any irreducible $\pi_{v}$ choose an orthonormal basis $\left\{\xi_{v}\right\}$, containing the $K_{v}$-fixed vector $\xi_{v}^{0}$ if $v \notin V$, the vector $\xi_{v_{1}}$ at $v=v_{1}$, and a basis of the space $\pi_{v}^{K_{1 v}}$ of $K_{1 v}$-fixed vectors for $v \in V_{1}$. Then $\{\phi\}=\left\{\otimes_{v} \xi_{v}\right\}\left(\xi_{v}=\xi_{v}^{0}\right.$ for all $\left.v \notin V\right)$ makes an orthonormal smooth basis of $\pi^{\mathbb{K}(V)}$.

If our global field is a function field, at each place $v \in V_{1}$ we let $f_{v}$ range only over the space of $K_{1 v}$-biinvariant functions. This we do in order to use Harish-Chandra's result that there exist only finitely many cuspidal representations with fixed infinitesimal characters at the archimedean places, and fixed ramification at all finite places. For such $f$ the sum over $\pi$ in (1) is then finite. If the base field is a number field we use spherical test functions and 
generalized linear independence of characters to obtain from [F8] the finiteness of the sum $(1)$.

Applying Bernstein's decomposition theorem ([B], see also [F4], p. 165), we may even choose the $f_{v}\left(v \in V_{1}\right)$ so that the components $\pi_{v}$ of the $\pi$ which occur in (1) have infinitesimal character in the same connected component as that of $\pi_{1 v}\left(v \in V_{1}\right)$; the terminology is that of $[\mathrm{B}]$ (and $[\mathrm{F} 4]$ ).

For each finite $v \in V_{1}$, the set of infinitesimal characters of the $\pi_{v}$ which occur in (1) is finite. Moreover, $\left\{\pi_{v}\left(f_{v}\right) ; f_{v} \in C_{c}^{\infty}\left(Z_{v} K_{1 v} \backslash G_{v} / K_{1 v}\right)\right\}$ is the algebra of endomorphisms of the space $\pi_{v}^{K_{1 v}}$ of $K_{1 v}$-fixed vectors in $\pi_{1 v}$. Consequently, if $\xi_{1 v}$ is the component at $v$ of $\phi_{i}(i=1,2)$, there is $f_{v}$ in $C_{c}^{\infty}\left(Z_{v} K_{1 v} \backslash G_{v} / K_{1 v}\right)$ such that $\pi_{v}\left(f_{v}\right)=0$ for each $\pi_{v} \nsucceq \pi_{1 v}$ which occurs in our sum, and such that $\pi_{1 v}\left(f_{v}\right)$ acts as 0 on each vector orthogonal to $\xi_{1 v}$, while $\pi_{1 v}\left(f_{v}\right) \xi_{1 v}=\xi_{2 v}$. Both sums over $\pi$ and $\phi$ reduce then to a single contribution, parametrized by $\pi_{1}$ and $\phi_{1}$, namely to

$$
W_{\psi}\left(\phi_{1}\right) P\left(\overline{\pi_{1}(f) \phi_{1}}\right)=W_{\psi}\left(\pi(s) \phi_{1}\right) P\left(\overline{\pi_{1}(f) \phi_{1}}\right) .
$$

Since $P\left(\pi_{1}(f) \phi_{1}\right)=P\left(\phi_{2}\right) \neq 0$, as $\phi_{2}$ is a cyclic vector, we conclude that $W_{\psi}\left(\pi(s) \phi_{1}\right)=$ $W_{\psi}\left(\phi_{1}\right)$ for all $s \in \operatorname{Stab}_{\mathbf{M}(\mathbb{A})}^{0}(\psi)$, in fact for any smooth cusp form $\phi_{1}$, as required.

Geometric conditions of Theorem A. Let us verify that Theorem A applies with the group $\mathbf{G}=\mathrm{GSp}(4)$ of section 2 . We need to determine a set of representatives $\{\delta\}$ for $N \backslash G / C$ such that $\psi_{T}$ is 1 on $\mathbf{N}(\mathbb{A}) \cap \delta \mathbf{C}(\mathbb{A}) \delta^{-1}$. By Proposition 1(b) such $\delta$ can be of the form $m$ or $m \gamma_{1}, m \in M$.

Singular case. Consider first $\delta=m \in M$. Then $\mathbf{N}(\mathbb{A}) \cap \delta \mathbf{C}(\mathbb{A}) \delta^{-1}$ consists of $\delta n \delta^{-1}, n=$ $\left(\begin{array}{ll}I & X \\ 0 & I\end{array}\right) \in \mathbf{C}(\mathbb{A})$, thus $X=\left(\begin{array}{ll}0 & y \\ z & 0\end{array}\right)$. Replacing $\varepsilon T$ by a conjugate if necessary we may assume, as in section 3 , that $\varepsilon T=\left(\begin{array}{ll}0 & 1 \\ \theta & 0\end{array}\right), \theta \in F-F^{2}$, or that $\varepsilon T=\left(\begin{array}{ll}u & 0 \\ 0 & v\end{array}\right), u, v \in F^{\times}, u-v \neq 0$. Since

$$
w^{t} U w\left(\begin{array}{cc}
0 & 1 \\
-\theta & 0
\end{array}\right) U X=\left(\begin{array}{ll}
d & b \\
c & a
\end{array}\right)\left(\begin{array}{cc}
c & d \\
-\theta a & -\theta b
\end{array}\right)\left(\begin{array}{ll}
0 & y \\
z & 0
\end{array}\right)=\left(\begin{array}{ll}
c d-a b \theta & d^{2}-\theta b^{2} \\
c^{2}-\theta a^{2} & c d-\theta a b
\end{array}\right)\left(\begin{array}{ll}
0 & y \\
z & 0
\end{array}\right) \quad\left(U=\left(\begin{array}{ll}
a & b \\
c & d
\end{array}\right)\right)
$$

has trace $\left(c^{2}-\theta a^{2}\right) y+\left(d^{2}-\theta b^{2}\right) z$, we have $\psi_{T}\left(\mathbf{N}(\mathbb{A}) \cap \delta \mathbf{C}(\mathbb{A}) \delta^{-1}\right)=1$ only if $c^{2}=\theta a^{2}$ and $d^{2}=\theta b^{2}$, contradicting the assumption that $\theta \in F$ is not a square.

When $T=\left(\begin{array}{cc}u & 0 \\ 0 & -v\end{array}\right)$ we need the coefficients of $z$ and $y$ in

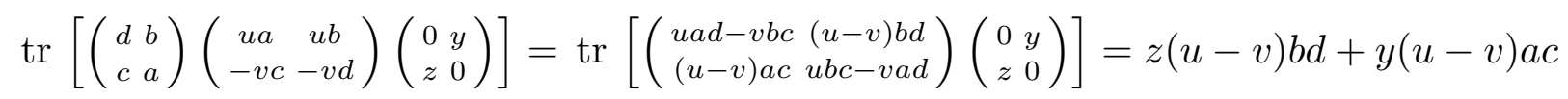

to vanish. Since $u \neq v$, we have $b d=0=a c$, and so $U=\left(\begin{array}{ll}a & 0 \\ 0 & d\end{array}\right)$ or $U=\left(\begin{array}{ll}0 & b \\ c & 0\end{array}\right)$. Since $\operatorname{Stab}_{\mathbf{M}(\mathbb{A})}^{0}\left(\psi_{T}\right)$ consists of $g=\operatorname{diag}(\alpha, \beta, \alpha, \beta)($ with $\lambda=\alpha \beta)$, we have $\delta^{-1} \operatorname{Stab}_{\mathbf{M}(\mathbb{A})}^{0}\left(\psi_{T}\right) \delta \subset$ $\mathbf{C}(\mathbb{A})$, which is the geometric requirement of the Theorem. 
Regular case. Next we consider the $\delta$ of the form $m \gamma, m \in M$. As noted in Proposition 1 , the image $\gamma J \Theta^{t} \gamma$ of $\gamma$ under the map $G / C \rightarrow X$ is $\left(\begin{array}{cc}-\omega & 0 \\ 0 & \omega\end{array}\right)$. The geometric requirement of the Theorem, that $\operatorname{Stab}_{\mathbf{M}(\mathbb{A})}^{0}(\psi)$ lies in $\delta \mathbf{C}(\mathbb{A}) \delta^{-1}$, follows from

$$
\frac{1}{\lambda} m \gamma J \Theta^{t} \gamma^{t} m=\frac{1}{\lambda}\left(\begin{array}{cc}
U & 0 \\
0 & \lambda w^{t} U^{-1} w
\end{array}\right)\left(\begin{array}{cc}
-\omega & 0 \\
0 & \omega
\end{array}\right)\left(\begin{array}{cc}
{ }^{t} U & 0 \\
0 & \lambda w U^{-1} w
\end{array}\right)=\left(\begin{array}{cc}
\frac{u}{\lambda} \omega^{-1} & 0 \\
0 & \frac{\lambda}{u} \omega
\end{array}\right),
$$

where $u=\operatorname{det} U$, since for any $T, \operatorname{Stab}_{\mathbf{M}(\mathbb{A})}^{0}(\psi)$ consists of $m=\operatorname{diag}\left(U, \lambda w^{t} U^{-1} w\right)$ with $\lambda=u$.

We shall consider next a similar example, where again $\mathbf{G}=\mathrm{GSp}(4)$, and $\mathbf{C}_{\tau}$ is the centralizer of $\Theta_{\tau}=\left(\begin{array}{cc}\boldsymbol{\tau} & 0 \\ 0 & \boldsymbol{\tau}\end{array}\right), \boldsymbol{\tau}=\left(\begin{array}{cc}0 & 1 \\ \tau & 0\end{array}\right)$, in $\mathbf{G}$, where $\tau \in F-F^{2}$.

To verify the geometric condition of the theorem, once again, we need to produce a set of representatives $\{\delta\}$ for $N \backslash G / C_{\tau}$ such that $\psi_{T}$ is 1 on $\mathbf{N}(\mathbb{A}) \cap \delta \mathbf{C}_{\tau}(\mathbb{A}) \delta^{-1}$. By Proposition 1 (c), such $\delta$ can be of the form $m$ or $m \gamma_{0}, m=m(U)=\operatorname{diag}\left(U, \lambda w^{t} U^{-1} w\right) \in M$.

Singular case. Suppose first that $\delta=m \in M$. Then $\mathbf{N}(\mathbb{A}) \cap \delta \mathbf{C}_{\tau}(\mathbb{A}) \delta^{-1}$ consists of $\delta n \delta^{-1}, n=\left(\begin{array}{ll}I & X \\ 0 & I\end{array}\right) \in \mathbf{C}_{\tau}(\mathbb{A})$, thus $X=\left(\begin{array}{cc}x & y \\ \tau & x\end{array}\right)$. As above we may assume that $\varepsilon T=$ $\left(\begin{array}{ll}0 & 1 \\ \theta & 0\end{array}\right), \theta \in F-F^{2}$, or that $\varepsilon T=\left(\begin{array}{ll}u & 0 \\ 0 & v\end{array}\right), u \neq v \in F^{\times}$; moreover, we take $\theta=\tau$ if $\theta \in \tau F^{\times 2}$. Since $\left(\right.$ for $\left.U=\left(\begin{array}{ll}a & b \\ c & d\end{array}\right)\right)$

$$
\operatorname{tr}\left[w^{t} U w\left(\begin{array}{rr}
0 & 1 \\
-\theta & 0
\end{array}\right) U X\right]=2(c d-a b \theta) x+\left[\left(c^{2}-\theta a^{2}\right)+\tau\left(d^{2}-\theta b^{2}\right)\right] y,
$$

$\psi_{T}\left(\mathbf{N}(\mathbb{A}) \cap \delta \mathbf{C}_{\tau}(\mathbb{A}) \delta^{-1}\right.$ ) can be 1 (for all $x, y$ ) only if $\theta / \tau \subset F^{\times 2}$, in which case we take $\theta=\tau$, and then $a= \pm d$ and $c= \pm \tau b$, namely $\delta=m(U)$ or $m(\varepsilon U)$, where $m(U) \in \operatorname{Stab}_{\mathbf{M}(\mathbb{A})}^{0}\left(\psi_{T}\right)$, and so $\delta^{-1} \operatorname{Stab}_{\mathbf{M}(\mathbb{A})}^{0}\left(\psi_{T}\right) \delta \subset \mathbf{C}_{\tau}(\mathbb{A})$, which is the geometric requirement of the Theorem.

When $\varepsilon T=\left(\begin{array}{ll}u & 0 \\ 0 & v\end{array}\right)$ we have $\operatorname{tr}\left(w^{t} U w\left(\begin{array}{ll}u & 0 \\ 0 & v\end{array}\right) U X\right)=(u-v)[x(a d+b c)+y(a c+b d \tau)]$, and it is clear that $\psi_{T}\left(\mathbf{N}(\mathbb{A}) \cap \delta \mathbf{C}_{\tau}(\mathbb{A}) \delta^{-1}\right)=1$ implies that $c^{2}=\tau d^{2}$, a contradiction.

Regular case. It remains to consider the $\delta$ of the form $m \gamma_{0}, m=m(U) \in M$. As $\lambda\left(\gamma_{0}\right)=1$, the image $\gamma_{0} \Theta_{\tau} J^{t} \gamma_{0}$ of $\gamma_{0}$ in $G / C_{\tau} \rightarrow X_{\tau}$ is $\left(\begin{array}{cc}\omega & 0 \\ 0 & -\tau \omega\end{array}\right)$, and once again, since for every $T$ the group $\operatorname{Stab}_{\mathbf{M}(\mathbb{A})}^{0}\left(\psi_{T}\right)$ consists of $m=\operatorname{diag}\left(U, \lambda w^{t} U^{-1} w\right)$ with $\lambda=\operatorname{det} U$, it is contained in $\delta \mathbf{C}_{\tau}(\mathbb{A}) \delta^{-1}$.

Remark. As mentioned above, any irreducible admissible $\mathrm{SO}\left(n, F_{v}\right)$-module $\pi_{v}$ has at most one - up to a scalar - $\mathrm{SO}\left(n-1, F_{v}\right)$-invariant linear form on its space (in characteristic zero). In our case $n=5$ and $G_{v}=\operatorname{PGSp}\left(4, F_{v}\right) \simeq \mathrm{SO}\left(5, F_{v}\right)$, and $C_{v}=\operatorname{SO}\left(4, F_{v}\right)$. Denote by $P_{v}$ such an invariant form on $\pi_{v}$, if it exists. Moreover, if $\pi_{v}$ is unramified we normalize $P_{v}$ to take the value 1 at the chosen $K_{v}$-invariant vector $\xi_{v}^{0}$. 
According to a theorem of Novodvorski and Piatetski-Shapiro [NPS], an irreducible $G_{v^{-}}$ module $\pi_{v}$ has at most one - up to a scalar - linear form $W_{\psi_{v}}$ which transforms via $W_{\psi_{v}}\left(\pi_{v}(s n) w\right)=\psi_{v}(n) W_{\psi_{v}}(w)$ for all $n \in N_{v}, s \in \operatorname{Stab}_{M_{v}}^{0}\left(\psi_{v}\right)$, and $w \in \pi_{v}$.

If $\pi=\otimes \pi_{v}$ is a cyclic cuspidal $\mathbf{G}(\mathbb{A})$-module, let $\left\{\xi_{v}\right\}$ denote an orthonormal basis of $\pi_{v}$ including the chosen $K_{v}$-fixed vector $\xi_{v}^{0}$ if $\pi_{v}$ is unramified, and consider the distribution

$$
f_{v} \mapsto\left(W_{\psi_{v}} \bar{P}_{v}\right)_{\pi_{v}}\left(f_{v}\right)=\sum_{\xi_{v}} W_{\psi_{v}}\left(\xi_{v}\right) P_{v}\left(\overline{\psi_{v}\left(f_{v}^{*}\right) \xi_{v}}\right)
$$

It is independent of the choice of the basis $\left\{\xi_{v}\right\}$, and for a set of inequivalent $\pi_{v}$, these distributions are linearly independent. If $\left(W_{\psi} \bar{P}\right)_{\pi}(f)=\sum_{\phi} W_{\psi}(\phi) P\left(\overline{\pi\left(f^{*}\right) \phi}\right)$ then there is a constant $c(\pi)$ such that for all $f=\otimes f_{v}$ we have a factorization

$$
\left(W_{\psi} \bar{P}\right)_{\pi}(f)=c(\pi) \prod_{v}\left(W_{\psi_{v}} \bar{P}_{v}\right)_{\pi_{v}}\left(f_{v}\right)
$$

\section{References}

[AAG] A. Aizenbud, N. Avni, D. Gourevitch, Spherical pairs over close local fields, arXiv:0910.3199.

[AGRS] A. Aizenbud, D. Gourevitch, S. Rallis, G. Schiffmann, Multiplicity one theorems, Ann. of Math. 172 (2010), 1407-1434.

[A1] J. Arthur, A trace formula for reductive groups. I. Terms associated to classes in $G(Q)$, Duke Math. J. 45 (1978), 911-952.

[A2] J. Arthur, A trace formula for reductive groups. II. Applications of a truncation operator, Compos. Math. 40 (1980), 87-121.

[A3] J. Arthur, On some problems suggested by the trace formula, in Lie group representations II, Springer Lecture Notes 1041 (1984), 209-249.

[A4] J. Arthur, An introduction to the trace formula, Clay Mathematics Proceedings, Volume 4, 2005.

[A5] J. Arthur, On the inner product of truncated Eisenstein series, Duke Math. J. 49 (1982), 35-70.

[BS] E. van den Ban, H. Schlichtkrull, Multiplicities in the Plancherel decomposition for a semi-simple symmetric space, in Representation theory of groups and algebras, Contemp. Math. 145 (1993), 163-180.

[B] J. Bernstein, Representations of p-adic groups Lectures by Joseph Bernstein. Written by Karl E. Rumelhart. Harvard University. (Fall 1992). \#48 in Publication List of http://www.math.tau.ac.il/ bernstei/

[Bo] A. Borel, Introduction aux groupes arithmétiques, Hermann, Paris 1969.

[BW] A. Borel, N. Wallach, Continuous Cohomology, Discrete Subgroups, and Representations of Reductive Groups, Ann. of Math Studies 94 (1980).

[F1] Y. Flicker, A Fourier summation formula for the symmetric space GL $(n) / \operatorname{GL}(n-1)$, Compos. Math. 88 (1993), 39-117. 
[F2] Y. Flicker, Cyclic automorphic forms on a unitary group, J. Math. Kyoto Univ. 37 (1997), 367-439.

[F3] Y. Flicker, Packets and liftings for U(3), J. Analyse Math. 50 (1988), 19-63.

[F4] Y. Flicker, On distinguished representations, J. reine angew. math. 418 (1991), 139-172.

[F5] Y. Flicker, Rigidity for automorphic forms, J. Analyse Math. 49 (1987), 135-202.

[F6] Y. Flicker, Regular trace formula and base change for GL(n), Ann. Inst. Fourier 40 (1990), 1-30.

[F7] Y. Flicker, Cusp forms on $\mathrm{GL}(2 n)$ with $\mathrm{GL}(n) \times \mathrm{GL}(n)$ periods, and simple algebras, Math. Nachrichten 183 (1997), 91-111.

[F8] Y. Flicker, Automorphic Forms and Shimura Varieties of PGSp(2), World Scientific, 2005.

[FH] Y. Flicker, J. Hakim, Quaternionic distinguished representations, Amer. J. Math. 116 (1994), 683-736.

[FK] Y. Flicker, D. Kazhdan, A simple trace formula, J. Analyse Math. 50 (1988), 189-200.

[G] R. Godement, Domaines fondamentaux des groupes arithmétiques, Sem. Bourbaki 257 (1963), 1-25.

[HCM] Harish-Chandra, Automorphic forms on semisimple Lie groups, Notes by J. G. M. Mars. Lecture Notes in Mathematics, No. 62 Springer-Verlag, Berlin-New York $1968 \mathrm{x}+138 \mathrm{pp}$.

[HPS] R. Howe, I. Piatetski-Shapiro, Some examples of automorphic forms on $\mathrm{Sp}(4)$, Duke Math. J. 50 (1983), 55-106.

[J1] H. Jacquet, Sur un résultat de Waldspurger, Ann. Sci. ENS. 19 (1986), 185-229.

[J2] H. Jacquet, On the nonvanishing of some $L$-functions, Proc. Indian Acad. Sci. 97 (1987), 117-155.

[J3] H. Jacquet, The continuous spectrum of the relative trace formula for GL(3) over a quadratic extention, Israel J. Math. 89 (1995), 1-59.

[J4] H. Jacquet, Automorphic spectrum of symmetric spaces, Proc. Symposia Pure Math. 61 (1997), 443-455.

[K] D. Kazhdan, Cuspidal geometry on p-adic groups, J. Analyse Math. 47 (1986), $1-36$.

[KR] S. Kudla, S. Rallis, Ramified degenerate principal series representations for $\operatorname{Sp}(n)$, Israel J. Math. 78 (1992), 209-256.

[La] S. Lang, Algebraic Number Theory, Addison-Wesley, 1970.

[MW] C. Moeglin, J.-L. Waldspurger, Spectral Decomposition and Eisenstein Series: Une Paraphrase de l'Ecriture, Cambridge Univ. Press, January 1995.

[NPS] M. Novodvorski, I. Piatetski-Shapiro, Generalized Bessel models for a symplectic group of rank 2, Math. USSR Sbornik 19 (1973), 243-255, 275-286, and 21 (1973), 499-509.

[O] T. Oda, On modular forms associated with indefinite quadratic forms of signature (2,n-2), Math. Ann. 231 (1977), 97-144. 
[OM] T. Oshima, T. Matsuki, A description of discrete series for semisimple symmetric spaces, Adv. Studies in Pure Math. 4 (1984), 331-390.

[PS1] I. Piatetski-Shapiro, On the Saito-Kurokawa lifting, Invent. Math. 71 (1983), 309-338; 76 (1984), 75-76.

[PS2] I. Piatetski-Shapiro, Special automorphic forms on PGSp(4), in Arithmetic and Geometry, Papers dedicated to I. R. Shafarevich, Progress in Math., PM 35, Birkhauser (1984), 309-325.

[PS3] I. Piatetski-Shapiro, $L$-functions for GSp(4), unpublished (1979).

[PR] V. Platonov, A. Rapinchuk, Algebraic groups and number theory, translated from the 1991 Russian original. Pure and Applied Mathematics, 139. Academic Press, Inc., Boston, MA, 1994. xii+614 pp.

[S] J.-P. Serre, Complex Semisimple Lie Algebras, Springer, New York, 1987.

[Sh] F. Shahidi, Langlands' conjecture on Plancherel measures for $p$-adic groups, in Harmonic Analysis on Reductive Groups (1991), 277-295.

[Sp] T. A. Springer, Some results on algebraic groups with involutions, in Algebraic Groups and Related Topics, Adv. Studies in Math. 6, Tokyo (1984), 323-343.

[SZ] B. Sun, C.-B. Zhu, A note on special orthogonal groups following Waldspurger, arXiv:1005.4484

[Wa1] J.-L. Waldspurger, Correspondence de Shimura, J. Math. Pure Appl. 59 (1980), $1-113$.

[Wa2] J.-L. Waldspurger, Sur les coefficients de Fourier des formes modulaires de poids demi-entier, J. Math. Pure Appl. 60 (1981), 375-484.

[Wa3] J.-L. Waldspurger, Une variante d'un résultat de Aizenbud, Gourevitch, Rallis et Schiffmann, http://arxiv.org/abs/0911.1618v1

[We] A. Weil, Sur certains groupes d'opérateurs unitaires, Acta Math. 111 (1964), 143-211; Sur la formule de Siegel dans la theorie des groupes classiques, Acta Math. 113 (1965), 1-87.

[Z] D. Zinoviev, Relation of orbital integrals on SO(5) and PGL(2), Israel J. Math. 106 (1998), 29-77. 